\title{
Evaluation of Defense-Waste Glass Produced by Full-Scale Vitrification Equipment
}

J. M. Lukacs

L. L. Petkus

G. B. Mellinger

September 1981

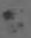

Prepared for the U.S. Department of Energy under Contract DE-AC06-76RLO 1830

Pacific Northwest Laboratory Operated for the U.S. Department of Energy by Battelle Memorial Institute 


\title{
NOTICE
}

This report was prepared as an account of work sponsored by the United States Government. Neither the United States nor the Department of Energy, nor any of their employees, nor any of their contractors, subcontractors, or their employees. makes any warranty, express or implied, or assumes any legal liability or responsibility for the accuracy, completeness or usefulness of any information, apparatus, product or process disclosed, or represents that its use would not infringe privately owned rights.

The views, opinions and conclusions contained in this report are those of the contractor and do not necessarily represent those of the United States Government or the United States Department of Energy.

\author{
PACIFIC NORTHWEST LABORATORY \\ operated by \\ BATTELLE \\ for the \\ UNITED STATES DEPARTMENT OF ENERGY \\ Under Contract DE-AC06-76RLO 1830
}

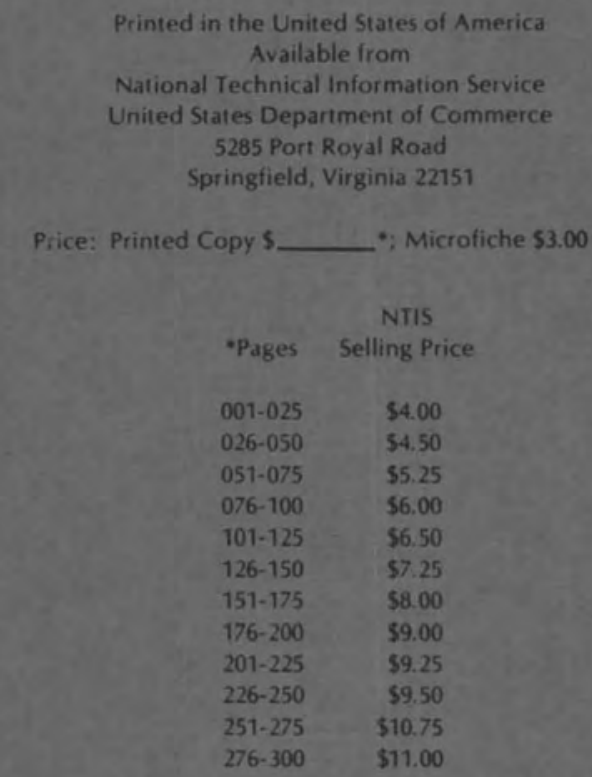




\section{6}

EVALUATION OF DEFENSE-WASTE GLASS

PRODUCED BY FULL-SCALE VITRIFICATION EQUIPMENT
J. M. Lukacs
L. L. Petkus
G. B. Mellinger

September 1981

Prepared for

the U.S. Department of Energy under Contract DE-AC06-76RLO 1830

Pacific Northwest Laboratory

Richland, Washington 99352 
SUMMARY

Three full-scale vitrification processes at the Pacific Northwest Laboratory produced over $67,000 \mathrm{~kg}$ of simulated nuclear-waste glass from March 1979 to August 1980. Samples were analyzed to monitor process operation and evaluate the resulting glass product. These processes are:

- Spray Calciner/In-Can Melter (SC/ICM)

- Spray Calciner/Calcine-Fed Ceramic Melter (SC/CFCM)

- Liquid-Fed Ceramic Melter (LFCM).

Waste components in the process feed varied less than $+10 \%$. The SC/ICM and SC/CFCM which use separate waste and frit feed systems showed larger glass compositional variation than the LFCM, which processed only premixed feed during this period. The SC/ICM and SC/CFCM product contained significant amounts of acmite crystals, while the LFCM product was largely amorphous. In addition, the lower portion of all SC/ICM-filled canisters contained a zone rich in waste components.

A product chemical durability as determined by $\mathrm{pH} 4$ and soxhlet leach tests varied considerably. Aside from increased durability under $\mathrm{pH} 4$ conditions with decreasing waste content, glass composition, microstructure and melting process did not correlate with glass durability. For all samples analyzed, the weight loss under $\mathrm{pH} 4$ conditions ranged from 17.7 to $85.2 \mathrm{wt} \%$. Soxhlet conditions produced weight losses from 1.78 to $3.56 \mathrm{wt} \%$. 
.

. 


\section{CONTENTS}

SUMMARY

FIGURES

TABLES

INTRODUCTION

WASTE GLASS COMPOSITION

PROCESS SAMPLING AND ANALYSIS

MELTING TRIALS .

SPRAY CALCINER/IN-CAN MELTER

FS-ICM-20 THROUGH FS-ICM-23 (MARCH 1979)

FS-ICM-24 (JULY 1979)

FS-ICM-26 AND FS-ICM-27 (MARCH 1980)

FS-ICM-28 AND FS-ICM-29 (AUGUST 1980)

LIQUID-FED CERAMIC MELTER.

SRL-13 (JULY 1979) .

SRL-14 (OCTOBER 1979)

SRL-LF-1 (JANUARY 1980)

SPRAY CALCINER/CALCINE-FED CERAMIC MELTER

CFCM-6 (APRIL 1979) .

CFCM-7 (JUNE 1979)

CFCM-8 (NOVEMBER 1979)

LIQUID FEED COMPOSITION .

MELTING PROCESS 


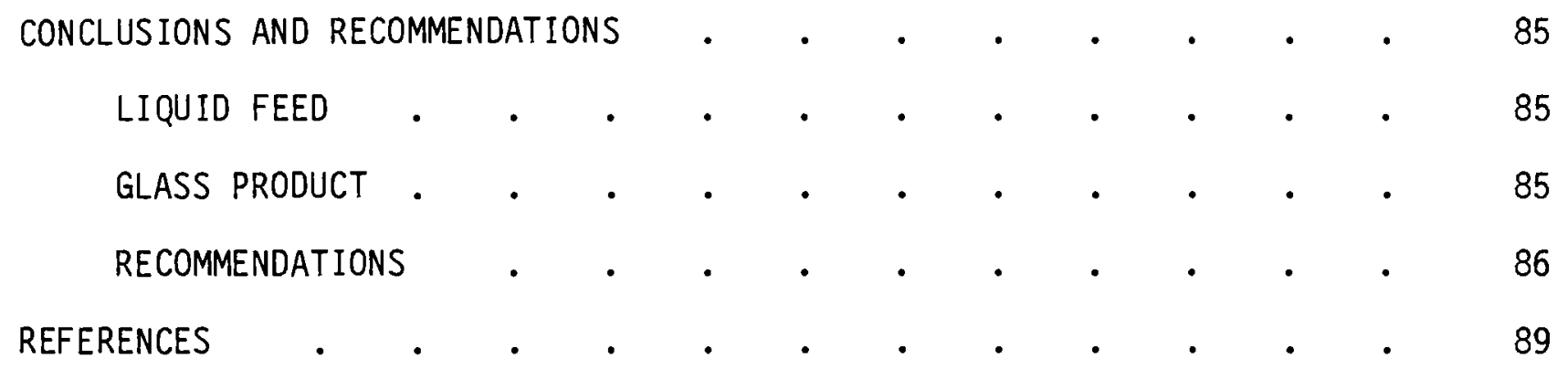




\section{FIGURES}

1 Spray Calciner/In-Can Melter . . . . . . . . . 10

2 Microstructure of Canister 177 Sample

[Height $0 \mathrm{~m}(0 \mathrm{ft})$; Depth $0-7.6 \mathrm{~cm}(0-3 \mathrm{in.})] . . .18$

3 Microstructure of Canister 177 Sample

[Height 0.61 m (2 ft); Depth 22.9-30.5 cm (9-12 in.)] . . . 19

4 Metallic Buttons Removed from Bottom of Canisters 172

5 Microstructure of Canister 174 Sample

[Height $0 \mathrm{~m}(0 \mathrm{ft})$; Depth 20.3 to $30.5 \mathrm{~cm}(8-12 \mathrm{in.})] .25$

6 Microstructure of Canister 172 Samples

[Height $0 \mathrm{~m}(0 \mathrm{ft})$; Depth 20.3 to $30.5 \mathrm{~cm}(8-12 \mathrm{in.})] .26$

7 Microstructure of Canister 174 Sample

[Height $0.61 \mathrm{~m}(2 \mathrm{ft})$; Depth 20.3 to $30.5 \mathrm{~cm}(8-12 \mathrm{in.})] .28$

8 Microstructure of Canister 172 Sample

[Height $0.61 \mathrm{~m}(2 \mathrm{ft})$; Depth 20.3-30.5 cm (8-12 in.)]. . .

9 Microstructure of Canister 174 Sample

[Height $1.21 \mathrm{~m}(4 \mathrm{ft}) ;$ Depth 0-10.2 cm (0-4 in.)] . . . 30

10 Microstructure of Canister 172 Sample

[Height $1.21 \mathrm{~m}(4 \mathrm{ft})$; Depth 0-10.2 cm $(0-4$ in. $)$ ] . . . 31

11 Liquid-Fed Ceramic Melter . . . . . . . . 36

12 TDS-211 Content of SRL-13 Grab Samples . . • • • . . 39

13 Total Oxide Content of CFCM-6 Liquid Feed . . . . . 53

14 Annealed Density of CFCM-6 Grab Samples. . . . . . . 53

15 Microstructure of Canister 92 Sample

[Height $0.30 \mathrm{~m}(1 \mathrm{ft})$; Depth-centerline] . . . . . 57

16 CaO Content of CFCM-7 Liquid Feed . . . . . . . 59

17 Annealed Density CFCM-7 Grab Samples . . • . . . 60

18 Microstructure of Canister 204 Sample

[Height $0.61 \mathrm{~m}(2 \mathrm{ft})$; Depth $11.4-22.9 \mathrm{~cm}(4.5-9.0$ in. $)$ ] . 
19 Microstructure of Canister 204 Sample

[Height $1.21 \mathrm{~m}(4 \mathrm{ft})$; Depth $11.4-22.9 \mathrm{~cm}(4.5-9.0$ in. $)$. . 68

$20 \mathrm{pH}$ of CFCM-8 Liquid Feed Samples . . . . . . . . 71

21 Microstructure of Canister 113 Sample

[Height $0.61 \mathrm{~m}(2 \mathrm{ft})$; Depth 22.9-30.5 cm $(9-12$ in. $)$. . . 75

22 Microstructure of Canister 113 Sample . . . . . . 76

23 Weight Loss of CFCM-8 Glass Samples Under pH4 Conditions . $\quad 78$ 


\section{$\underline{\text { TABLES }}$}

1 Simulated TDS Liquid Feed Composition Used in Equipment Tests Runs

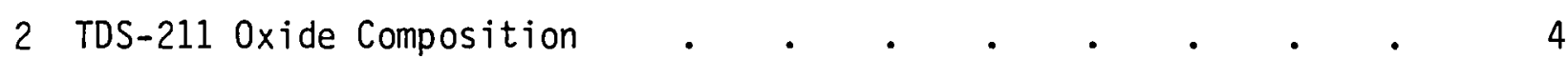

3 Melting Trial Sample Analysis . . . . . . . . . 6

4 PNL Defense Waste Melting Trials . $\quad . \quad$. $\quad . \quad$. 7

5 SC/ICM Melting Trials . . . . . . . . . . . 11

6 Normalized Composition of Canister 175 Samples . . . . . 12

7 Normalized Composition of Canister 179 Samples . . . . 12

8 Crystalline Content of Canister 175 and 179 Samples . . . 13

9 Soxhlet Leach Data of Canister 175 and 179 Samples . . . 14

10 Normalized Composition of Canister 177 Samples . • • . $\quad$ • 15

11 Average Compositions of Canister 177 Samples . . . . . 16

12 Crystalline Content of Canister 177 Samples . . . . . . 17

13 Weight Loss of Canister 177 Samples Under pH4 Conditions . . 20

14 Weight Loss of Canister 177 Samples Under Soxhlet Conditions .

15 Normalized Composition of Canister 174 Samples $\quad$ • . . $\quad$ • 22

16 Normalized Composition of Canister 172 Samples . • • . $\quad 22$

17 Average Composition of Canister 174 Samples . . . . . 23

18 Average Composition of Canister 172 Samples . . . . . 24

19 Crystalline Content of Canister 174 Samples . . . . . . 27

20 Crystalline Content of Canister 172 Samples . . . . . 27

21 Weight Loss of Canister 174 Samples Under pH4 Conditions . • 32

22 Weight Loss of Canister 174 Samples Under Soxhlet

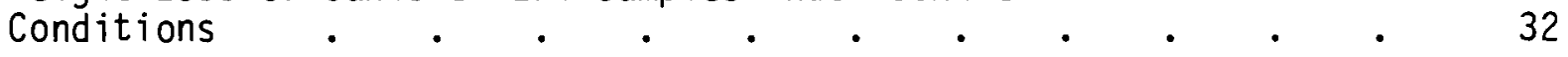

23 Weight Loss of Canister 172 Samples Under pH4 Conditions . . 33 
24 Weight Loss of Canister 172 Samples Under Soxhlet Conditions

25 Normalized Composition SRL-13 Grab Samples . . . . . . 38

26 Estimated TDS-211 Composition . . . . . . . . 40

27 Normalized Composition Of Canister 148 Samples . . . . 40

28 Average Composition of Canister 148 Samples . . . . . 41

29 Weight Loss of Canister 148 Sample Under pH4 Conditions . . 41

30 Weight Loss of Canister 148 Sample Under Soxhlet Conditions . . 42

31 SRL-LF-1 Liquid Feed Makeup . . . . . . . . . . 43

32 Composition of SRL-LF-1 Liquid Feed Samples . • • . . 44

33 Normalized Composition of SRL-LF-1 Grab Samples . . . . . 46

34 Normalized Composition of Canister 10 Samples . . . . 47

35 Weight Loss of Canister 10 Samples Under pH4 Conditions . • 47

36 Weight Loss of Canister 10 Samples Under Soxhlet Conditions . $\quad 47$

37 Composition of CFCM-6 Liquid Feed Samples, g 0xide/L Feed . • 51

38 Normalized Composition of CFCM-6 Grab Samples . . . . 54

39 Crystalline Content of Canister 92 Samples . . . . . . 58

40 Weight Loss of Canister 92 Samples Under pH4 Conditions . . 58

41 Weight Loss of Canister 92 Samples Under Soxhlet Conditions . • 58

42 Normalized Composition of Pour Glass Samples During SRL-LF-1 • 61

43 Normalized Composition of CFCM-7 Grab Samples . . . . . 62

44 Correlation Coefficients of Glass Density and Composition . . 64

45 Normalized Composition of Canister 204 Samples . . . . . 65

46 Average Composition of Canister 204 Samples . . . . . . 65

47 Crystalline Content of Canister 204 Samples . . . . . . 66 
48 Weight Loss of Canister 204 Samples Under pH4 Conditions . .

49 Weight Loss of Canister 204 Samples Under Soxhlet Conditions

50 Composition of CFCM-8 Liquid Feed Samples . . . . . 70

51 Normalized Composition of CFCM-8 Grab Samples . . . . 72

52 Normalized Composition of Canister 113 Samples • • • • $\quad 73$

53 Average Composition of Canister 113 Samples . . . . . 74

54 Crystalline Content of Canister 113 Samples . • • • • 77

55 Weight Loss of Canister 113 Samples Under pH4 Conditions . . 77

56 Weight Loss of Canister 113 Samples Under Soxhlet

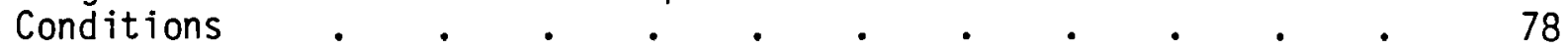

57 Data Summary for Melting Trial Samples . . . • . $\quad$ • 80

58 Summary of SC/CFCM Liquid Feed Analysis, g Oxide/L Feed . . 81 
.

. 


\section{INTRODUCTION}

The safe disposal of nuclear waste is essential for the continued use of nuclear energy in the United States and abroad. A key element in proposed waste-management systems is solidification of nuclear wastes in an inert form. One technique that has been extensively investigated is the vitrification of wastes to produce a stable and durable waste glass. Researchers at the Pacific Northwest Laboratory (PNL), operated by Battelle Memorial Institute for the Department of Energy (DOE), have been active in the development of both glass compositions and process equipment for the production of nuclear waste glasses. Researchers at PNL have designed, constructed and operated full-scale vitrification equipment to evaluate three waste vitrification processes:

- Spray Calciner/In-Can Melter (SC/ICM)

- Spray Calciner/Calcine-Fed Ceramic Melter (SC/CFCM)

- Liquid-Fed Ceramic Melter (LFCM).

This equipment is capable of filling canisters 2.1 to $3.0 \mathrm{~m}(7$ to $10 \mathrm{ft}$ ) tall with molten, simulated nuclear-waste glass which solidifies on cooling.

The Savannah River Laboratory (SRL), operated by E. I. DuPont de Nemours for the Department of Energy (DOE), is developing technology to immobilize and encapsulate nuclear waste sludges stored at the Savannah River Plant (SRP). The major process under consideration is the vitrification of SRP waste in borosilicate glass. The Pacific Northwest Laboratory supported this effort by conducting test runs with SRL-developed glasses in its full-scale melting equipment. Equipment runs of up to 25 continuous days of operation evaluated many aspects of equipment design, process operation and processibility of anticipated SRP wastes and waste glasses.

The waste glass compositions used in these equipment test runs were developed to be compatible with the vitrification processes and to meet durability criteria for the eventual disposal of nuclear-waste glass. During PNL test runs, feed and product samples were collected to evaluate the effect of the process on waste-glass composition and properties. Each of the three vitrification processes will be described along with the data collected during each melting trial. 



\section{WASTE GLASS COMPOSITION}

Since March 1979, melting trials at PNL have processed a simulated TDS-211 waste glass composition. Premelted and ground 211 frit was melted with simulated TDS waste in a 72/28 oxide weight ratio. The TDS waste stream simulates the anticipated waste composition during actual operation at SRP. Table 1 lists the constituents used to produce simulated TDS liquid feed for PNL tests. The overall concentration was altered in various runs to improve system operation. The defined oxide composition of TDS calcine, 211 frit and TDS-211 glass are listed in Table 2. Later melting trials added minor constituents such as $\mathrm{Cl}^{-}$, $\mathrm{RuO}_{2}, \mathrm{Sb}_{2} \mathrm{O}_{3}$ and $\mathrm{SrO}$, or organic components such as $\mathrm{Na}_{4}$ EDTA, cornstarch and anthracite coal to the liquid feed to produce a more accurate liquid-waste stream simulation. These modifications did not significantly alter final waste or waste-glass oxide compositions.

TABLE 1. Simulated TDS Liquid Feed Composition Used in Equipment Test Runs

\begin{tabular}{|c|c|c|}
\hline $\begin{array}{c}\text { Chemical } \\
\text { Constituents }\end{array}$ & $\begin{array}{l}\text { Defined TDS } \\
\text { Waste, } g / L\end{array}$ & $\begin{array}{l}\text { Modified TDS } \\
\text { Waste, } g / L\end{array}$ \\
\hline $\mathrm{Fe}(\mathrm{OH})_{3}$ & 35.1 & 100.3 \\
\hline $\mathrm{Al}_{2} \mathrm{O}_{3} 3 \mathrm{H}_{2} \mathrm{O}$ & 8.8 & 25.2 \\
\hline $\mathrm{MnO}_{2}$ & 7.0 & 20.0 \\
\hline $\mathrm{CaCO}_{3}$ & 3.2 & 9.1 \\
\hline $\mathrm{NiO} \quad 2 \mathrm{H}_{2} \mathrm{O}$ & 4.5 & 12.9 \\
\hline $\mathrm{NaNO}_{3}$ & 1.5 & 4.2 \\
\hline $\mathrm{Na}_{2} \mathrm{SO}_{4}$ & 0.7 & 2.0 \\
\hline Zeolite(a) & 5.2 & 1.5 \\
\hline Cornstarch & --- & 0.8 \\
\hline $\mathrm{Na}_{4}$ EDTA & -- & 11.6 \\
\hline \multirow[t]{2}{*}{ Anthracite Coal } & $\cdots$ & 4.2 \\
\hline & 66.0 & 191.8 \\
\hline
\end{tabular}

(a) Linde Ionsiv 95 
TABLE 2. TDS-211 0xide Composition

\begin{tabular}{|c|c|c|c|c|}
\hline \multirow[b]{2}{*}{$\begin{array}{c}\text { Oxide } \\
\text { Constituents } \\
\end{array}$} & \multicolumn{4}{|c|}{ Oxide wt\% } \\
\hline & 211 Frit & Zeolite (a) & IDS Waste & $\begin{array}{c}\text { TDS-211 } \\
\text { Waste Glass } \\
\end{array}$ \\
\hline $\mathrm{Fe}_{2} \mathrm{O}_{3}$ & --- & 4.0 & 52.6 & 14.7 \\
\hline $\mathrm{Al}_{2} \mathrm{O}_{3}$ & --- & 17.1 & 13.2 & 3.7 \\
\hline $\mathrm{MnO}_{2}$ & --- & 0.1 & 13.9 & 3.9 \\
\hline $\mathrm{CaO}^{2}$ & 5.6 & 3.9 & 4.0 & 5.1 \\
\hline $\mathrm{NiO}$ & --- & --- & 6.1 & 1.7 \\
\hline $\mathrm{Na}_{2} \mathrm{O}$ & 20.6 & 4.4 & 0.7 & 15.3 \\
\hline $\mathrm{Na}_{2} \mathrm{SO}_{4}$ & --- & -- & 0.9 & $0.4^{(b)}$ \\
\hline $\mathrm{SiO}_{2}$ & 58.3 & 68.6 & 7.2 & 44.0 \\
\hline $\mathrm{LiO}_{2}$ & 4.4 & -- & -- & 3.2 \\
\hline $\mathrm{B}_{2} \mathrm{O}_{3}$ & 11.1 & --- & --- & 8.0 \\
\hline & 100.0 & 98.1 & 98.6 & 100.0 \\
\hline
\end{tabular}

(a) Linde Ionsiv-95 - $1.9 \%$ ignition loss $800^{\circ} \mathrm{C}$

(b) $\mathrm{SO}_{3}^{-}$ 


\section{PROCESS SAMPLING AND ANALYSIS}

A11 PNL full-scale vitrification processes can convert simulated waste and frit into a borosilicate glass. Samples of the liquid waste, frit, waste-glass product and offgas were collected and analyzed during melting trials. This information was used to evaluate process stability, behavior of waste glass constituents during processing and properties of solidified waste glass.

The specific samples collected and the sampling frequency varied with the type of vitrification equipment operated and the run objectives. Liquid feed samples were collected directly upstream from the spray calciner or LFCM in 100-cc poylethylene bottles. Glass samples were either collected directly from the pouring spout of the LFCM or CFCM or core drilled from specific locations in the filled canisters after cooling. The sampling and analysis of the offgas stream during melting trials is not dealt with in this report.

Several analytical techniques were used to monitor process operation and waste glass properties. Those used during the course of SRL melting trials are listed in Table 3. Sample chemical analysis was done exclusively with the Induction Coupled Plasma Spectrophotometer (ICP). Chick et al. (1980) describes in detail the procedure used for ICP chemical analysis and x-ray diffraction crystal content analysis. Glass density which is very sensitive to chemical composition and thermal history was determined by the Archimedes technique. Each density data point was the average for three samples which were first annealed at $500^{\circ} \mathrm{C}$ for $2 \mathrm{~h}$ and slow cooled to eliminate thermal history effects.

Chemical durability was determined by soxhlet and pH4 leach tests. Onegram samples $(-40+60$ mesh) were placed in stainless steel mesh envelopes and leached in distilled water at $90^{\circ} \mathrm{C}$ for $24 \mathrm{~h}$ (soxhlet test) and in an agitated $\mathrm{pH} 4$ acidic solution for $19 \mathrm{~h}$. The weight loss of the sample as a result of leaching was determined. Reported data represents the average values for two samples.

All analytical techniques described were not applied to each sample collected. The object of most analytical work was to monitor process operation 


\section{TABLE 3. Melting Trial Sample Analysis}

\begin{tabular}{|c|c|c|}
\hline $\begin{array}{l}\text { Process } \\
\text { Sample }\end{array}$ & Data Collected & Analytical Technique \\
\hline Liquid Feed & $\begin{array}{l}\text { Specific gravity } \\
\text { pH } \\
\text { Oxide content } \\
\text { Oxide composition }\end{array}$ & $\begin{array}{l}\text { Measure weight and volume } \\
\text { Bechman } 3500 \mathrm{pH} \text { meter } \\
\text { Dried } 125^{\circ} \mathrm{C} \text {; fired } 800^{\circ} \mathrm{C} \\
\text { Induction Coupled } \\
\text { Plasma Spectrophotometer } \\
\text { (ICP) }\end{array}$ \\
\hline Frit & $\begin{array}{l}\text { Density } \\
\text { Oxide composition }\end{array}$ & $\begin{array}{l}\text { Archimedes technique } \\
\text { ICP }\end{array}$ \\
\hline Waste Glass & $\begin{array}{l}\text { Density } \\
\text { Oxide composition } \\
\text { Chemical durability } \\
\text { Crystal content }\end{array}$ & $\begin{array}{l}\text { Archimedes technique } \\
\text { ICP } \\
\text { Soxhlet and } \mathrm{pH} 4 \text { leach } \\
\text { test } \\
\text { X-ray diffraction }\end{array}$ \\
\hline
\end{tabular}

with a reasonable amount of laboratory support. Sampling frequency and laboratory analyses were modified by both experience and unanticipated events during the melting trials. Canister samples were taken and analyzed for SRL.

\section{MELTING TRIALS}

From March 1979 to August 1980, 10 melting trials were run with a simulated TDS-211 glass in the three vitrification processes. During $63 \mathrm{~d}$ of operation, 44 canisters were filled with over $67,000 \mathrm{~kg}$ of waste glass. Several defense waste-glass melting trials were also conducted in the Experimental Ceramic Melter (ECM) and Alternative Waste Form Melter (AWFM). Details regarding these trials are not included because of their development objectives and lower operating capacities. Table 4 is a chronological listing of the full-scale equipment trials conducted during this period. The sample data collected will be reported separately for the SC/ICM, SC/CFCM and LFCM processes. A brief description of each process will preceed each set of data. 
TABLE 4. PNL Defense Waste Melting Trials (March 1979 - August 1980)

\begin{tabular}{l} 
Melting Trial \\
Identification \\
\hline FS-ICM-20 through \\
FS-ICM-23 \\
CFCM-6 \\
CFCM-7 \\
FS-ICM-24 \\
SRL-13 \\
SRL-14 \\
CFCM-8 \\
SRL-LF-1 \\
FS-ICM-26 and \\
FS-ICM- 27 \\
FS-ICM-28 and \\
FS-ICM-29 \\
TOTAL
\end{tabular}

\begin{tabular}{|c|c|c|c|c|}
\hline Process & Trial Date & $\begin{array}{c}\text { Trial } \\
\text { Duration, } \\
\mathrm{d}\end{array}$ & $\begin{array}{l}\text { Number of } \\
\text { Canisters } \\
\end{array}$ & $\begin{array}{c}\text { Waste } \\
\text { Glass, } \\
\mathrm{kg} \\
\end{array}$ \\
\hline $\mathrm{SC} / \mathrm{ICM}$ & March 1979 & 5 & 4 & 4,415 \\
\hline $\mathrm{SC} / \mathrm{CFCM}$ & April 1979 & 10 & 7 & 11,000 \\
\hline $\mathrm{SC} / \mathrm{CFCM}$ & June 1979 & 25 & 18 & 35,000 \\
\hline $\mathrm{SC} / \mathrm{ICM}$ & July 1979 & 1 & 1 & 1,202 \\
\hline LFCM & July 1979 & 5 & 3 & 4,250 \\
\hline LFCM & September 1979 & 3 & 1 & 2,480 \\
\hline SC/CFCM & November 1979 & 5 & & \\
\hline LFCM & January 1980 & 3 & 2 & 1,050 \\
\hline $\mathrm{SC} / \mathrm{ICM}$ & March 1980 & 2 & 2 & 2,160 \\
\hline \multirow[t]{2}{*}{$\mathrm{SC} / \mathrm{ICM}$} & August 1980 & 4 & 2 & 1,900 \\
\hline & & 63 & 44 & 67,657 \\
\hline
\end{tabular}




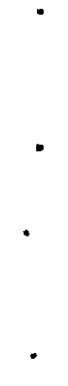




\section{SPRAY CALCINER/IN-CAN MELTER}

The Spray Calciner/In-Can Melter (SC/ICM) process was developed at PNL to convert a liquid nuclear-waste stream into a durable glass. Bonner (1976) and Blair (1979) describe in detail the design, construction and operation of the SC/ICM. Simulated aqueous nuclear waste is pumped into a spray nozzle at the top of the calciner. Compressed air atomizes the liquid waste into fine droplets which fall through the externally heated calciner walls. Radiant energy $\left(600^{\circ} \mathrm{C}\right.$ to $\left.800^{\circ} \mathrm{C}\right)$ quickly evaporates the water and partially calcines the waste oxides. Most of the calcine drops free of the water vapor and other gases at the bottom of the calciner; the remainder is removed from the offgas stream by sintered metal filters. These filters are periodically cleaned by a reverse pulse of compressed air which disperses the collected calcine to the bottom of the calciner. The glass-forming constituents are added to the process as a premelted, preground glass frit. This frit is continuously metered into the calciner barrel where it falls with the calcine through the bottom of the calciner into a heated $\left(1050^{\circ} \mathrm{C}\right.$ to $\left.1150^{\circ} \mathrm{C}\right)$ canister which functions as a melting crucible. The arrangement of the PNL SC/ICM process is shown in Figure 1.

Normal operating canister wall temperatures are $1075^{\circ} \mathrm{C}$ for stainless steel and $1150^{\circ} \mathrm{C}$ for Inconel canisters. Corresponding melt temperatures may be $25^{\circ}$ to $50^{\circ} \mathrm{C}$ cooler. As more calcine and frit are added, the level of molten glass rises. All SC/ICM canisters filled with defense waste glass from March 1979 to March 1980 were $0.61 \mathrm{~m}$ (24 in.) in dia and $2.13 \mathrm{~m}$ (84 in.) tall. When the canister has cooled to room temperature, the glass product can be sampled by core-drilling at specific intervals. The analysis of these samples will be presented separately for each of the three melting trials conducted during this period (Table 5).

FS-ICM-20 THROUGH FS-ICM-23 (MARCH 1979)

During March 1979 the SC/ICM was operated for $5 \mathrm{~d}$, producing TDS-211 waste glass. The objectives of this melting trial were to demonstrate long-term 


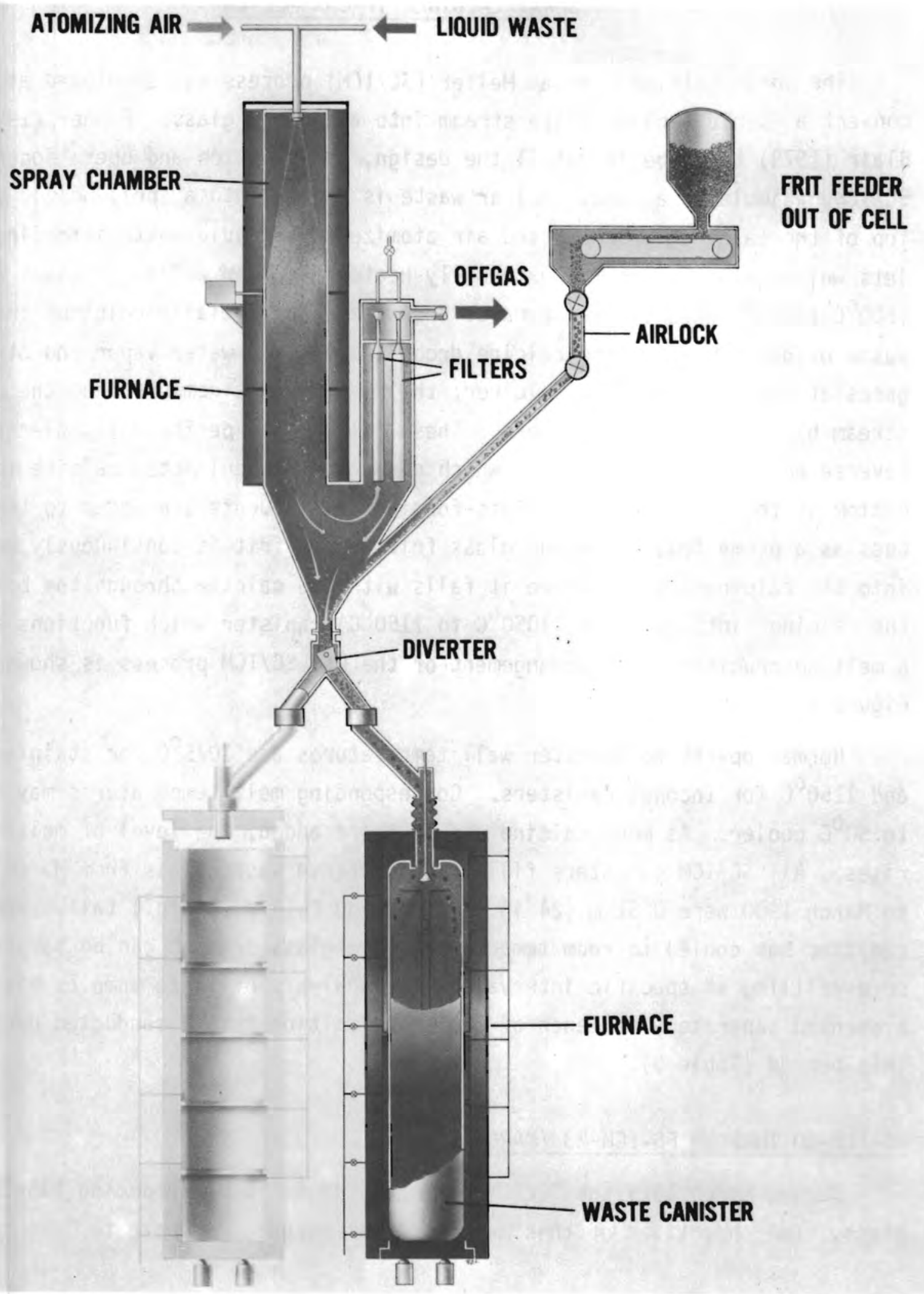

FIGURE 1. Spray Calciner/In-Can Melter 
TABLE 5. SC/ICM Melting Trials (April 1979 - April 1980)

\begin{tabular}{|c|c|c|c|c|c|c|c|}
\hline \multirow{2}{*}{$\begin{array}{c}\text { Melting } \\
\text { Trial } \\
\end{array}$} & \multirow[b]{2}{*}{ Canister } & Trial & \multicolumn{2}{|c|}{$\begin{array}{l}\text { Maximum Glass } \\
\text { Melting Rate }\end{array}$} & \multicolumn{2}{|c|}{$\begin{array}{l}\text { Average } \\
\text { Melting }\end{array}$} & \multirow{2}{*}{$\begin{array}{c}\text { Glass } \\
\text { Produced, } \\
\mathrm{kg} \\
\end{array}$} \\
\hline & & Date & $\mathrm{kg} / \mathrm{h}$ & $\mathrm{kg} / \mathrm{h} / \mathrm{m}^{2}$ & $\mathrm{~kg} / \mathrm{h}$ & $\mathrm{kg} / \mathrm{h} / \mathrm{m}^{2}$ & \\
\hline $\begin{array}{r}\text { FS-ICM- } 20 \\
-21 \\
-22 \\
-23\end{array}$ & $\begin{array}{l}175^{(a)} \\
178 \\
176 \\
179\end{array}$ & March 1979 & $\begin{array}{r}>75 \\
>75 \\
>85 \\
80\end{array}$ & $\begin{array}{r}>286 \\
>286 \\
>324 \\
305\end{array}$ & $\begin{array}{l}59 \\
47 \\
35 \\
46\end{array}$ & $\begin{array}{l}225 \\
179 \\
134 \\
176\end{array}$ & $\begin{array}{l}1107 \\
1089 \\
1075 \\
1144\end{array}$ \\
\hline FS-ICM-24 & $177^{(\mathrm{a})}$ & July 1979 & 60 & 229 & 45 & 172 & 1202 \\
\hline $\begin{array}{r}F S-I C M-26 \\
-27\end{array}$ & $\begin{array}{l}174(a)(b) \\
172(a)(b)\end{array}$ & March 1980 & $\begin{array}{l}75 \\
74\end{array}$ & $\begin{array}{l}286 \\
282\end{array}$ & $\begin{array}{l}75 \\
74\end{array}$ & $\begin{array}{l}286 \\
282\end{array}$ & $\begin{array}{l}1136 \\
1024\end{array}$ \\
\hline $\begin{array}{r}F S-I C M-28 \\
-29\end{array}$ & $\begin{array}{l}180(a)(b) \\
181^{(a)(b)}\end{array}$ & August 1980 & $\begin{array}{l}85 \\
20\end{array}$ & $\begin{array}{r}324 \\
76\end{array}$ & $\begin{array}{l}85 \\
20\end{array}$ & $\begin{array}{r}324 \\
76\end{array}$ & $\begin{array}{r}1169 \\
805\end{array}$ \\
\hline
\end{tabular}

(a) Canisters core-drilled for product analysis.

(b) TDS waste composition modified with organic compounds. Glass oxide composition unchanged.

operation of SC/ICM equipment, processing cycle times and maximum melting rates for the TDS-211 waste glass composition. Four canisters with internal heat transfer fins were filled with a total of $4415 \mathrm{~kg}$ of waste glass. The calcine/ frit mixture melted rapidly without foaming. Calcine, however, tended to accumulate on top of the fin assembly, altering material distribution. After filling, each canister contained 2 to $5 \mathrm{~kg}$ of loose calcine material which was not incorporated into the glass. The maximum sustained melting rate was $85 \mathrm{~kg} / \mathrm{h}\left(324 \mathrm{~kg} / \mathrm{h} / \mathrm{m}^{2}\right)$. The average melting rate for each canister was 35 to $60 \mathrm{~kg} / \mathrm{h}\left(134\right.$ to $\left.229 \mathrm{~kg} / \mathrm{h} / \mathrm{m}^{2}\right)$.

Samples from the first (175) and fourth (179) canisters were core-drilled for analysis. The normalized analytical results in Tables 6 and 7 show that significant compositional variation occurred both within and between canisters. The lower $0.30 \mathrm{~m}(1 \mathrm{ft})$ of both canisters was enriched in calcine constituents, $\mathrm{Fe}_{2} \mathrm{O}_{3}, \mathrm{MnO}_{2}$ and $\mathrm{NiO}$, containing approximately 46 wt\% calcine oxides versus the defined $28 \mathrm{wt} \%$. The estimated calcine content in the remainder of both canisters was low, 24 wt\% in canister 175 and 19 wt\% in canister 179 with a standard 
TABLE 6. Normalized Composition of Canister 175 Samples

Core

Height

Oxide wt\%

\begin{tabular}{|c|c|c|c|c|c|c|c|c|c|c|}
\hline \multicolumn{2}{|c|}{$\mathrm{m} \quad \mathrm{ft}$} & $\overline{\mathrm{SiO}_{2}}$ & $\underline{A l}_{2}-{ }_{-3}$ & $\underline{B}_{2} \underline{-}_{3}$ & $\mathrm{CaO}(\mathrm{b})$ & $\underline{\mathrm{Fe}}_{2} \underline{-}_{3}$ & $\underline{L i}_{2}$ & $\underline{\mathrm{Na}}_{2} \underline{O}$ & $\mathrm{MnO}_{2}$ (b) & $\underline{\mathrm{NiO}}$ \\
\hline \multicolumn{2}{|c|}{ Defined } & 44.0 & 3.7 & 8.0 & 5.1 & 14.7 & 3.2 & 15.5 & 3.9 & 1.7 \\
\hline 0.06 & 0.2 & 31.1 & 8.3 & 5.3 & & 32.4 & & 5.9 & & 3.8 \\
\hline 0.15 & 0.5 & 34.9 & 7.0 & 5.9 & & 28.6 & & 7.5 & & 3.1 \\
\hline 0.30 & 1 & 45.8 & 3.3 & 8.1 & & 18.5 & & 10.2 & & 1.1 \\
\hline 0.61 & 2 & 48.5 & 2.6 & 8.6 & & 15.5 & & 10.6 & & 1.1 \\
\hline 0.91 & 3 & 48.2 & 2.6 & 8.5 & & 15.5 & & 11.0 & & 1.2 \\
\hline 0.21 & 4 & 46.4 & 2.8 & 8.4 & & 12.4 & & 15.7 & & 1.2 \\
\hline \multicolumn{2}{|c|}{$\begin{array}{l}\text { AVE. (a) } \\
\text { STD. DEV. }\end{array}$} & $\begin{array}{r}47.2 \\
1.3\end{array}$ & $\begin{array}{l}2.8 \\
0.3\end{array}$ & $\begin{array}{l}8.4 \\
0.2\end{array}$ & & $\begin{array}{r}15.4 \\
2.5\end{array}$ & & $\begin{array}{r}11.9 \\
2.6\end{array}$ & & $\begin{array}{l}1.2 \\
0.1\end{array}$ \\
\hline
\end{tabular}

(a) Data from lower $0.30 \mathrm{~m}(1 \mathrm{ft}$ ) omitted from calculated averages.

(b) Component analys is not reported.

TABLE 7. Normalized Composition of Canister 179 Samples

Core

$\frac{\text { Height }}{\mathrm{m} \quad \mathrm{ft}}$

Defined

$0.06 \quad 0.25$

$0.15 \quad 0.5$

0.612

0.913

1.214

1.525

AVE. (a)

STD. DEV .

$$
\mathrm{SiO}
$$

44.

31.7

31.

45.0

45.3

45.8

45.8

46.7

45.7

$45.7 \quad 3.0$

0.70 .2

7.4

5.7

5.7

9.0

2.8

8.9

9.1

9.1

9.0

0.1
Oxide wt\%

$\underline{\mathrm{Fe}}_{2} \underline{O}_{3} \quad \underline{\mathrm{Li}}_{2} \underline{\mathrm{O}} \underline{\mathrm{b}}_{\mathrm{b}} \quad \underline{\mathrm{Na}}_{2} \underline{\mathrm{O}} \quad \underline{\mathrm{MnO}}_{2} \underline{\mathrm{(b})} \quad \underline{\mathrm{NiO}}$

$14.7 \quad 3.2$

15.5

3.9

1.7

27.4

11.8

2.9

27.4

11.8

2.9

12.2

16.3

1.2

12.5

16.6

1.0

11.2

16.9

1.1

10.2

17.0

1.1

11.5

1.0

16.7

1.1

0.1

(a) Data from lower $0.30 \mathrm{~m}$ ( $1 \mathrm{ft}$ ) omitted from calculated averages.

(b) Component analys is not reported. 
deviation of \pm 2 wt\%. Liquid-feed system problems encountered frequently during this run could account for the higher compositional variation noted in canister 175 .

The calcine-rich glass in the bottom of both canisters contained 29 to 35 wt\% of spinel crystals suspended in a glass matrix. Only acmite crystal phases were found above the $0.30-\mathrm{m}(1-\mathrm{ft})$ level. The quantitative crystal content data is presented in Table 8 . Glass near the canister centerline cooled more slowly $\left(\approx 25^{\circ} \mathrm{C} / \mathrm{h}\right)$ than that near the wall $\left(70\right.$ to $\left.90^{\circ} \mathrm{C} / \mathrm{h}\right)$. Slower cooling rates can produce more devitrification and higher crystal contents. While canister 179 shows this behavior, canister 175 does not. Soxhlet leach data $(72 \mathrm{~h})$ shown in Table 9 does not indicate a strong relationship between soxhlet leach rate and acmite crystal content. Under soxhlet conditions, the calcinerich glass containing spinel crystals had a lower weight loss than for the rest of the glass, $4.6 \mathrm{wt} \%$ versus $9.7 \pm 2.4$ wt\%.

TABLE 8. Crystalline Content of Canister 175 and 179 Samples

\begin{tabular}{|c|c|c|c|c|c|}
\hline \multirow{2}{*}{ Canister } & \multirow{2}{*}{\multicolumn{2}{|c|}{ Core Height }} & \multicolumn{2}{|c|}{$\begin{array}{c}\text { Crystalline } \\
\text { Component wt\% }\end{array}$} & \multirow{2}{*}{$\begin{array}{c}\text { Crystalline } \\
\text { Phases Present }\end{array}$} \\
\hline & & & $\begin{array}{c}\text { Canister } \\
\text { Wall }\end{array}$ & $\begin{array}{l}\text { Canister } \\
\text { Centerline }\end{array}$ & \\
\hline \multirow{5}{*}{175} & 0 & 0 & --- & $35 \%$ & Spine ${ }^{(a)}$ \\
\hline & 0.30 & 1 & $14 \%$ & $14 \%$ & Acmite \\
\hline & 0.61 & 2 & $16 \%$ & $10 \%$ & Acmite \\
\hline & 0.91 & 3 & $13 \%$ & $13 \%$ & Acmite \\
\hline & 1.21 & 4 & $27 \%$ & $28 \%$ & Acmite \\
\hline \multirow{6}{*}{179} & 0 & 0 & -- & $29 \%$ & Spinel \\
\hline & 0.30 & 1 & $28 \%$ & $28 \%$ & Acmite \\
\hline & 0.61 & 2 & $11 \%$ & $28 \%$ & Acmite \\
\hline & 0.91 & 3 & $0 \%$ & $15 \%$ & Acmite \\
\hline & 1.21 & 4 & $0 \%$ & $26 \%$ & Acmite \\
\hline & 1.52 & 5 & $0 \%$ &.-- & --- \\
\hline
\end{tabular}

(a) Spinel ( $\mathrm{Ni}, \mathrm{Mn}) \mathrm{Fe}_{2} \mathrm{O}_{4}$ 
TABLE 9. Soxhlet Leach Data of Canister 175 and 179 Samples

\begin{tabular}{|c|c|c|c|c|}
\hline \multirow[b]{2}{*}{ Canister } & \multirow{2}{*}{\multicolumn{2}{|c|}{ Core 1}} & \multicolumn{2}{|c|}{ wt\% Loss } \\
\hline & & $\frac{g h t}{f t}$ & $\begin{array}{c}\text { Canister } \\
\text { Wall }\end{array}$ & $\begin{array}{l}\text { Canister } \\
\text { Center line }\end{array}$ \\
\hline & 0 & 0 & --- & 4.5 \\
\hline & 0.30 & 1 & 9.2 & 12.5 \\
\hline 175 & 0.61 & 2 & 11.6 & 11.9 \\
\hline & 0.91 & 3 & 10.0 & 9.0 \\
\hline & 1.21 & 4 & 12.8 & 11.1 \\
\hline $\begin{array}{l}\text { AVE. } \\
\text { STD. DEV. }\end{array}$ & & & $\begin{array}{r}10.9 \\
1.6\end{array}$ & $\begin{array}{r}11.0 \\
1.4\end{array}$ \\
\hline
\end{tabular}

\begin{tabular}{cllcc} 
& 0 & 0 & --- & 4.8 \\
\multirow{3}{*}{179} & 0.30 & 1 & 13.4 & 11.0 \\
& 0.61 & 2 & 6.5 & 10.6 \\
& 0.91 & 3 & 6.2 & 7.3 \\
& 1.21 & 4 & 6.7 & 8.1 \\
& 1.52 & 5 & 6.6 & --- \\
AVE. & & & 7.9 & 9.2 \\
STD. DEV. & & & 1.3 & 1.8
\end{tabular}

\section{FS-ICM-24 (JULY 1979)}

The objective of the second TOS-211 melting trial in the SC/ICM was to establish the maximum melting rate in a canister without heat transfer fins and evaluate the resulting waste glass product. Canister 177 was filled with $1202 \mathrm{~kg}$ of glass at a maximum rate of $65 \mathrm{~kg} / \mathrm{h}\left(248 \mathrm{~kg} / \mathrm{h} / \mathrm{m}^{2}\right)$ with an average rate of $45 \mathrm{~kg} / \mathrm{h}\left(172 \mathrm{~kg} / \mathrm{h} / \mathrm{m}^{2}\right)$. No melting problems were encountered; however, the canister contained $8 \%$ less glass than anticipated.

Core samples taken at various heights and depths from canister 177 were analyzed. Chemical composition as a function of location is shown in Table 10 and summarized in Table 11. Oniy one sample taken from the bottom wall of the 
TABLE 10. Normalized Composition of Canister 177 Samples

\begin{tabular}{|c|c|c|c|c|c|c|c|c|c|c|c|c|}
\hline \multicolumn{2}{|c|}{$\begin{array}{c}\text { Core } \\
\text { Height }\end{array}$} & \multicolumn{2}{|c|}{ Core Depth } & \multicolumn{9}{|c|}{ Oxide wt\% } \\
\hline$m$ & $\underline{\mathrm{ft}}$ & $\mathrm{cm}$ & in. & $\underline{\mathrm{SiO}} 2$ & A) $2 \underline{O}_{3}$ & $\underline{B}_{2} \underline{-}_{3}$ & $\mathrm{CaO}$ & $\mathrm{Fe}_{2} \underline{-}_{3}$ & $\underline{L i}_{2} \underline{-}$ & $\underline{\mathrm{Na}} 2 \underline{O}$ & $\mathrm{MnO}_{2}$ & NiO \\
\hline Defir & & & & 44.0 & 3.7 & 8.0 & 5.1 & 14.7 & 3.2 & 15.5 & $3.9^{\circ}$ & 1.7 \\
\hline 0 & 0 & $\begin{array}{l}0-7.6 \\
7.6-15.2 \\
15.2-22.9 \\
22.9-30.5\end{array}$ & $\begin{array}{l}0-3 \\
3-6 \\
6-9 \\
9-12\end{array}$ & $\begin{array}{l}31.7 \\
45.7 \\
48.8 \\
48.3\end{array}$ & $\begin{array}{l}6.4 \\
4.1 \\
3.2 \\
3.3\end{array}$ & $\begin{array}{l}5.6 \\
8.0 \\
8.8 \\
8.7\end{array}$ & $\begin{array}{l}4.6 \\
5.2 \\
5.1 \\
5.0\end{array}$ & $\begin{array}{l}25.8 \\
13.1 \\
10.3 \\
10.6\end{array}$ & $\begin{array}{l}2.1 \\
3.0 \\
3.5 \\
3.2\end{array}$ & $\begin{array}{l}13.4 \\
15.1 \\
15.4 \\
15.9\end{array}$ & $\begin{array}{l}6.4 \\
3.9 \\
3.1 \\
3.1\end{array}$ & $\begin{array}{l}3.5 \\
1.4 \\
1.3 \\
1.3\end{array}$ \\
\hline 0.30 & 1 & $\begin{array}{l}0-7.6 \\
7.6-15.2 \\
15.2-22.9 \\
22.9-30.5\end{array}$ & $\begin{array}{l}0-3 \\
3-6 \\
6-9 \\
9-12\end{array}$ & $\begin{array}{l}49.3 \\
49.2 \\
49.6 \\
49.0\end{array}$ & $\begin{array}{l}3.1 \\
3.1 \\
3.0 \\
3.1\end{array}$ & $\begin{array}{l}8.9 \\
8.8 \\
8.9 \\
8.8\end{array}$ & $\begin{array}{l}5.1 \\
5.1 \\
5.1 \\
5.2\end{array}$ & $\begin{array}{r}9.2 \\
9.3 \\
9.2 \\
10.2\end{array}$ & $\begin{array}{l}3.6 \\
3.4 \\
3.6 \\
3.5\end{array}$ & $\begin{array}{l}16.3 \\
16.5 \\
15.9 \\
15.3\end{array}$ & $\begin{array}{l}2.8 \\
2.9 \\
2.8 \\
3.1\end{array}$ & $\begin{array}{l}1.2 \\
1.2 \\
1.2 \\
1.3\end{array}$ \\
\hline 0.61 & 2 & $\begin{array}{l}0-7.6 \\
7.6-15.2 \\
15.2-22.9 \\
22.9-30.5\end{array}$ & $\begin{array}{l}0-3 \\
3-6 \\
6-9 \\
9-12\end{array}$ & $\begin{array}{l}50.0 \\
49.6 \\
49.4 \\
48.4\end{array}$ & $\begin{array}{l}3.0 \\
3.0 \\
3.1 \\
3.2\end{array}$ & $\begin{array}{l}9.0 \\
9.0 \\
8.9 \\
8.7\end{array}$ & $\begin{array}{l}5.3 \\
5.0 \\
5.0 \\
4.9\end{array}$ & $\begin{array}{l}9.6 \\
9.0 \\
9.2 \\
9.6\end{array}$ & $\begin{array}{l}3.4 \\
3.4 \\
3.7 \\
3.8\end{array}$ & $\begin{array}{l}15.0 \\
16.4 \\
16.2 \\
16.8\end{array}$ & $\begin{array}{l}3.0 \\
2.8 \\
2.8 \\
2.9\end{array}$ & $\begin{array}{l}1.3 \\
1.2 \\
1.2 \\
1.2\end{array}$ \\
\hline 0.91 & 3 & $\begin{array}{l}0-7.6 \\
7.6-15.2 \\
15.2-22.9 \\
22.9-30.5\end{array}$ & $\begin{array}{l}0-3 \\
3-6 \\
6-9 \\
9-12\end{array}$ & $\begin{array}{l}49.8 \\
49.7 \\
49.5 \\
49.2\end{array}$ & $\begin{array}{l}3.0 \\
3.0 \\
3.0 \\
3.1\end{array}$ & $\begin{array}{l}8.9 \\
8.9 \\
9.0 \\
8.7\end{array}$ & $\begin{array}{l}5.2 \\
5.2 \\
5.2 \\
5.0\end{array}$ & $\begin{array}{l}9.3 \\
9.5 \\
9.5 \\
9.4\end{array}$ & $\begin{array}{l}3.4 \\
3.5 \\
3.5 \\
3.2\end{array}$ & $\begin{array}{l}15.8 \\
15.5 \\
15.5 \\
16.8\end{array}$ & $\begin{array}{l}2.9 \\
2.9 \\
3.0 \\
2.9\end{array}$ & $\begin{array}{l}1.2 \\
1.3 \\
1.3 \\
1.3\end{array}$ \\
\hline 1.21 & 4 & $\begin{array}{l}0-7.6 \\
7.6-15.2 \\
15.2-22.9\end{array}$ & $\begin{array}{l}0-3 \\
3-6 \\
6-9\end{array}$ & $\begin{array}{l}49.5 \\
49.5 \\
49.5\end{array}$ & $\begin{array}{l}3.0 \\
3.0 \\
3.0\end{array}$ & $\begin{array}{l}8.8 \\
8.8 \\
8.9\end{array}$ & $\begin{array}{l}5.1 \\
5.1 \\
5.1\end{array}$ & $\begin{array}{l}9.3 \\
9.2 \\
9.4\end{array}$ & $\begin{array}{l}3.5 \\
3.6 \\
3.6\end{array}$ & $\begin{array}{l}16.1 \\
16.0 \\
15.8\end{array}$ & $\begin{array}{l}2.9 \\
2.9 \\
2.9\end{array}$ & $\begin{array}{l}1.2 \\
1.2 \\
1.3\end{array}$ \\
\hline 1.52 & 5 & $\begin{array}{l}0-7.6 \\
7.6-15.2\end{array}$ & $\begin{array}{l}0-3 \\
3-6\end{array}$ & $\begin{array}{l}49.3 \\
49.7\end{array}$ & $\begin{array}{l}3.0 \\
3.0\end{array}$ & $\begin{array}{l}8.8 \\
8.8\end{array}$ & $\begin{array}{l}4.9 \\
5.1\end{array}$ & $\begin{array}{l}8.9 \\
9.2\end{array}$ & $\begin{array}{l}3.8 \\
3.2\end{array}$ & $\begin{array}{l}16.7 \\
16.3\end{array}$ & $\begin{array}{l}2.8 \\
2.9\end{array}$ & $\begin{array}{l}1.3 \\
1.2\end{array}$ \\
\hline
\end{tabular}

canister contained an estimated 49 wt\% calcine similar to the previous SC/ICM trial. The bulk glass produced during this trial contained only 21 wt\% calcine compared to the target value of $28 \mathrm{wt} \%$. This descrepancy which could have resulted from either a reduced feed concentration or a higher frit feed rate largely accounts for the lower than anticipated glass production. There was little compositional variation throughout the canister from top to bottom or from wall to centerline.

The crystal content of canister 177 listed in Table 12 was comparable to that noted previously in canister 179 . There was a limited distribution of spinel in the bottom (maximum $40 \mathrm{wt} \%$ ). (Figure 2 is a photomicrograph of this unhomogeneous spinel-rich zone on the floor of the canister. The small bright crystals are spinel phase.) Acmite was found primarily along the bottom and 
TABLE 11. Average Compositions of Canister 177 Samples(a)

Core Height

\begin{tabular}{|c|c|c|c|c|c|c|c|c|c|c|}
\hline & \\
\hline $\mathrm{m}$ & $\mathrm{ft}$ & $\overline{\mathrm{SiO}_{2}} 2$ & $\mathrm{Al}_{2}{ }^{0} \underline{3}_{3}$ & $\bar{B}_{2}-{ }_{-}-3$ & $\mathrm{CaO}$ & $\mathrm{Fe}_{2} \underline{O}_{3}$ & $\underline{L i}_{2} \underline{0}$ & $\underline{\mathrm{Na}}_{2} \underline{0}$ & $\mathrm{MnO}_{2}$ & $\mathrm{NiO}$ \\
\hline Defined & & 44.0 & 3.6 & 8.0 & 5.0 & 14.0 & 3.2 & 16.0 & 3.9 & 1.7 \\
\hline 0 & 0 & 43.6 & 4.2 & 7.8 & 5.0 & 15.0 & 3.0 & 15.0 & 4.1 & 1.9 \\
\hline 0.30 & 1 & 49.3 & 3.1 & 8.8 & 5.1 & 9.5 & 3.5 & 16.0 & 2.9 & 1.2 \\
\hline 0.61 & 2 & 49.4 & 3.1 & 8.9 & 5.0 & 9.4 & 3.6 & 16.1 & 2.9 & 1.2 \\
\hline 0.91 & 3 & 49.6 & 3.0 & 8.9 & 5.2 & 9.4 & 3.4 & 15.9 & 2.9 & 1.3 \\
\hline 1.21 & 4 & 49.5 & 3.0 & 8.8 & 5.1 & 9.3 & 3.6 & 16.0 & 2.9 & 1.2 \\
\hline 1.52 & 5 & 49.5 & 3.0 & 8.8 & 5.0 & 9.0 & 3.5 & 16.5 & 2.8 & 1.2 \\
\hline
\end{tabular}

Oxide wt\% 
TABLE 12. Crystalline Content of Canister 177 Samples

\begin{tabular}{|c|c|c|c|c|c|}
\hline \multirow{2}{*}{\multicolumn{2}{|c|}{$\begin{array}{c}\text { Core } \\
\text { Height }\end{array}$}} & \multicolumn{4}{|c|}{ Core Depth, cm (in.) } \\
\hline & $\mathrm{ft}$ & $\begin{array}{l}0-7.6 \\
(0-3)\end{array}$ & $\begin{array}{c}7.6-15.2 \\
(3-6)^{2}\end{array}$ & $\begin{array}{c}15.2-22.9 \\
(6-9)\end{array}$ & $\begin{array}{c}22.9-30.5 \\
(9-12)\end{array}$ \\
\hline 0 & 0 & $30-40 \%$ Spine ${ }^{(a)}$ & $\begin{array}{l}\text { 5-10\% Spinel } \\
5-10 \% \text { Acmite }^{(b)}\end{array}$ & $\begin{array}{l}10-15 \% \text { Acmite } \\
\text { 22\% Spinel }\end{array}$ & $\begin{array}{r}25 \% \text { Acmite } \\
2 \% \text { Spinel }\end{array}$ \\
\hline 0.30 & 1 & 0 & 0 & 0 & $25 \%$ Acmite \\
\hline 0.61 & 2 & 0 & 0 & 0 & 10-15\% Acmite \\
\hline 0.91 & 3 & 0 & $<5 \%$ Acmite & $5 \%$ Acmite & $10 \%$ Acmite \\
\hline 1.21 & 4 & 0 & 0 & 0 &.-- \\
\hline 1.52 & 5 & 0 & 0 & -- & --- \\
\hline
\end{tabular}

(a) Spinel - ( $\mathrm{Ni}, \mathrm{Mn}) \mathrm{Fe}_{2} \mathrm{O}_{4}$

(b) Acmite - $\mathrm{NaFe}\left(\mathrm{SiO}_{3}\right)_{2}$

\section{FS-ICM-26 AND FS-ICM-27 (MARCH 1980)}

The object of this melting trial was to assess the impact of organic additions in the waste stream on process equipment operation and waste glass properties. The new waste composition referred to as "modified TDS" incorporates three organic compounds, $\mathrm{Na}_{4}$ EDTA, cornstarch, and anthracite coal in the TDS waste (Table 1). McElroy (1979, p. 25) showed that carbon compounds such as coal may not be significantly altered by calcination. At glass-melting temperatures they are oxidized, reducing the oxidation state of metal oxide waste constituents in the glass.

The March $1980 \mathrm{SC} / \mathrm{ICM}$ melting trial filled two finless canisters with $2160 \mathrm{~kg}$ of modified TDS-211 waste glass. The primary changes between the two canisters was the operating temperature of the ICM furnace. The first canister, 174 , was run at $1075^{\circ} \mathrm{C}$, which is considered the ICM reference operating temperature. The second canister, 172, was fabricated from Inconel and run at $1150^{\circ} \mathrm{C}$. Canister 174 was filled at approximtely $75 \mathrm{~kg} / \mathrm{h}\left(286 \mathrm{~kg} / \mathrm{h} / \mathrm{m}^{2}\right)$. Production was not limited by foaming, and no unmelted material was noted on the surface of the glass. Canister 172 was to be filled at $100 \mathrm{~kg} / \mathrm{h}\left(382 \mathrm{~kg} / \mathrm{h} / \mathrm{m}^{2}\right)$ 


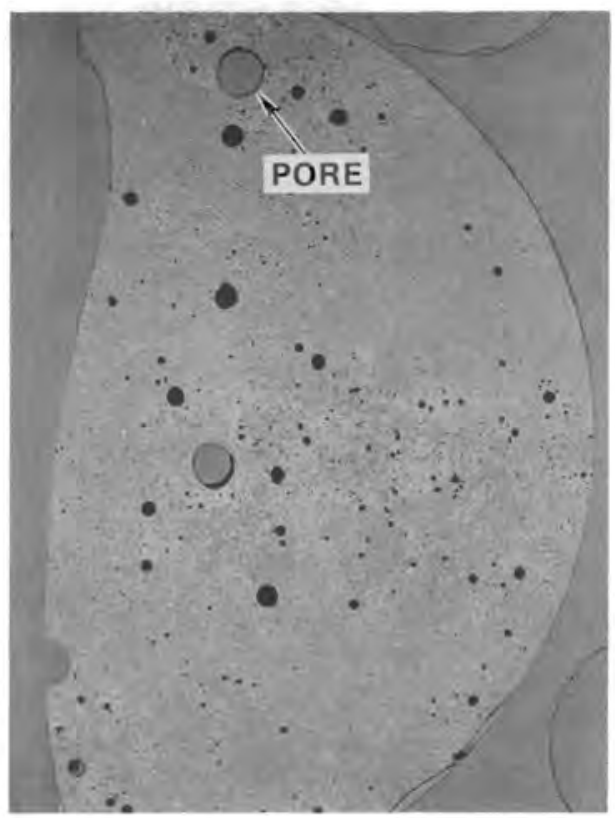

$5.2 X$
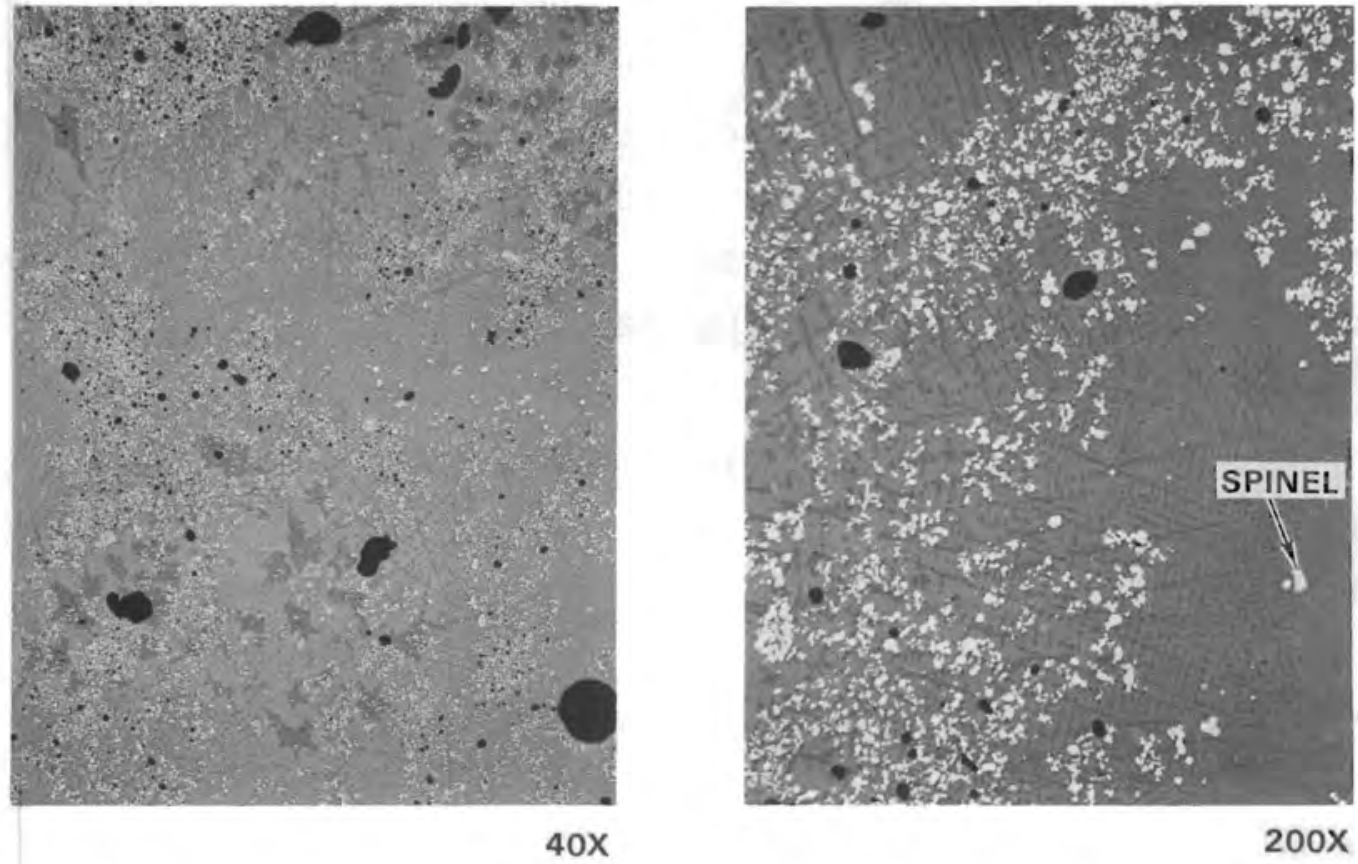

$200 x$

FIGURE 2. Microstructure of Canister 177 Sample [Height $0 \mathrm{~m}(0 \mathrm{ft})$; Depth 0-7.6 cm (0-3 in.)] 


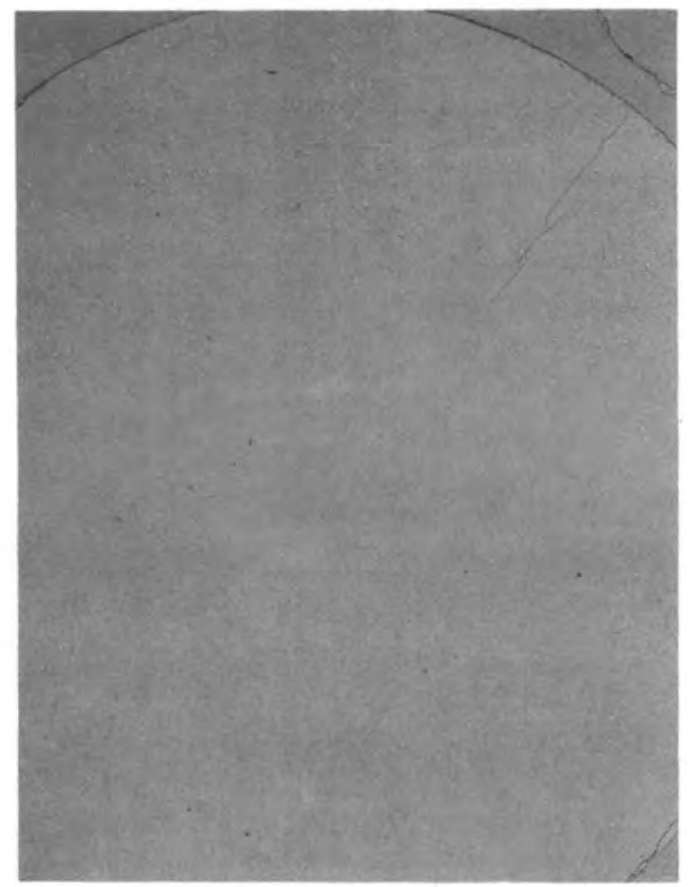

$5.2 \mathrm{X}$
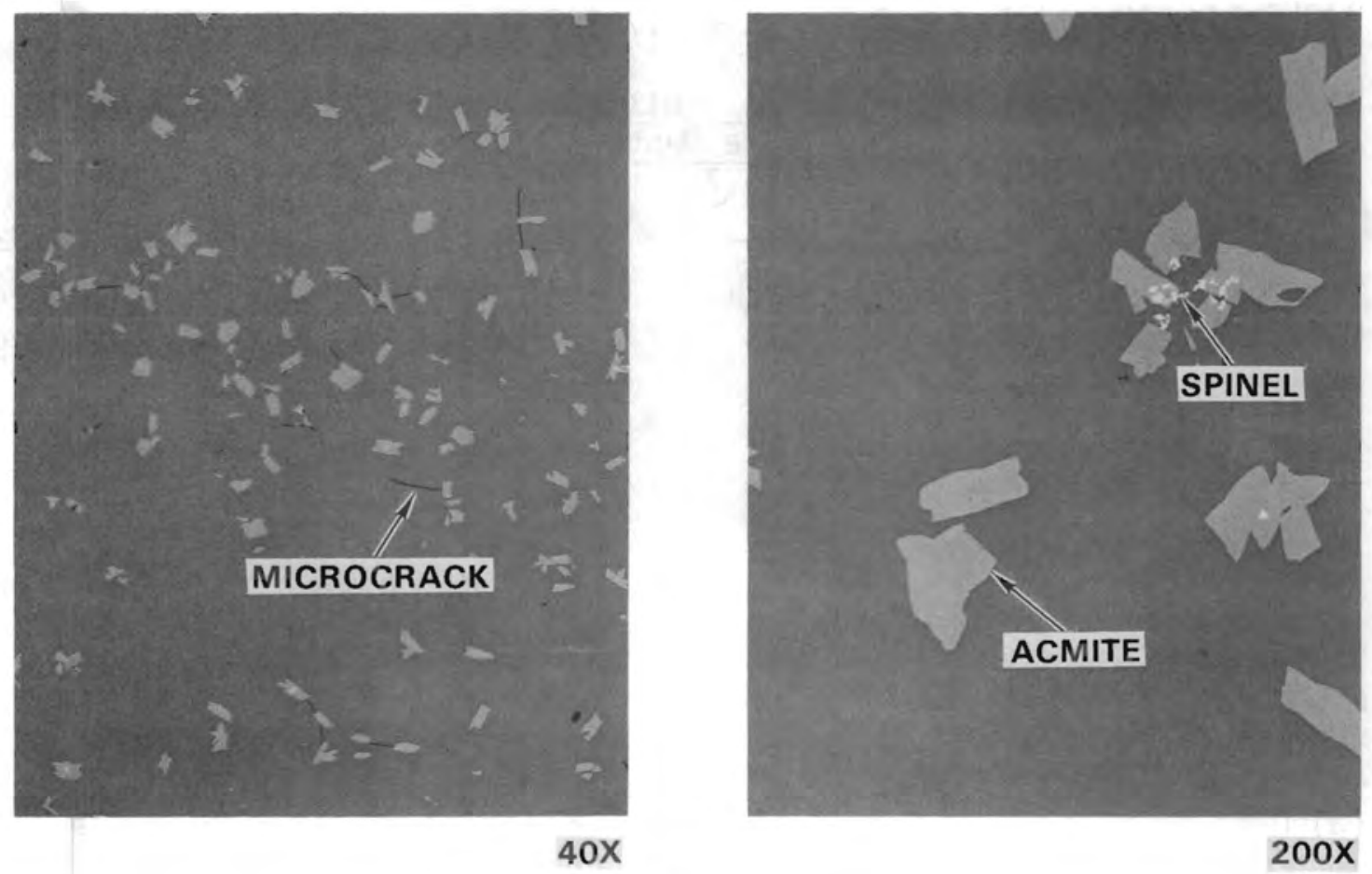

FIGURE 3. Microstructure of Canister 177 Sample [Height $0.61 \mathrm{~m}(2 \mathrm{ft})$; Depth 22.9-30.5 cm (9-12 in.)] 
TABLE 13. Weight Loss of Canister 177 Samples Under pH4 Conditions

\begin{tabular}{|c|c|c|c|c|c|c|}
\hline \multirow[b]{2}{*}{$\frac{\text { Core }}{m}$} & \multirow[b]{2}{*}{$\frac{\text { Height }}{f t}$} & \multicolumn{4}{|c|}{ wt\% Loss } & \\
\hline & & $\begin{array}{l}0-7.6 \\
(0-3) \\
\end{array}$ & $\begin{array}{c}\text { Core } \\
7.6-15.2 \\
(3-6)\end{array}$ & $\begin{array}{c}7, \mathrm{~cm} \text { (in. } \\
15.2-22.9 \\
(6-9)\end{array}$ & $\begin{array}{c}22.9-30.5 \\
(9-12) \\
\end{array}$ & Ave \\
\hline 0 & 0 & --- & 55.8 & 51.2 & 34.9 & 47.3 \\
\hline 0.30 & 1 & 11.7 & 15.0 & 9.8 & 31.1 & 17.2 \\
\hline 0.61 & 2 & 16.7 & 12.0 & 14.5 & 35.3 & 19.6 \\
\hline 0.91 & 3 & 13.4 & 21.2 & 18.6 & 20.3 & 8.4 \\
\hline 1.21 & 4 & 17.1 & 16.6 & 16.3 & --- & 16.8 \\
\hline 1.52 & 5 & 18.6 & 14.5 & -- & -- & 16.5 \\
\hline AVE. & & 15.5 & 15.9 & 14.9 & 28.9 & \\
\hline
\end{tabular}

(a) Data from lower $0.30-\mathrm{m}(1-\mathrm{ft})$ level omitted from calculated averages.

TABLE 14. Weight Loss of Canister 177 Samples Under Soxhlet Conditions

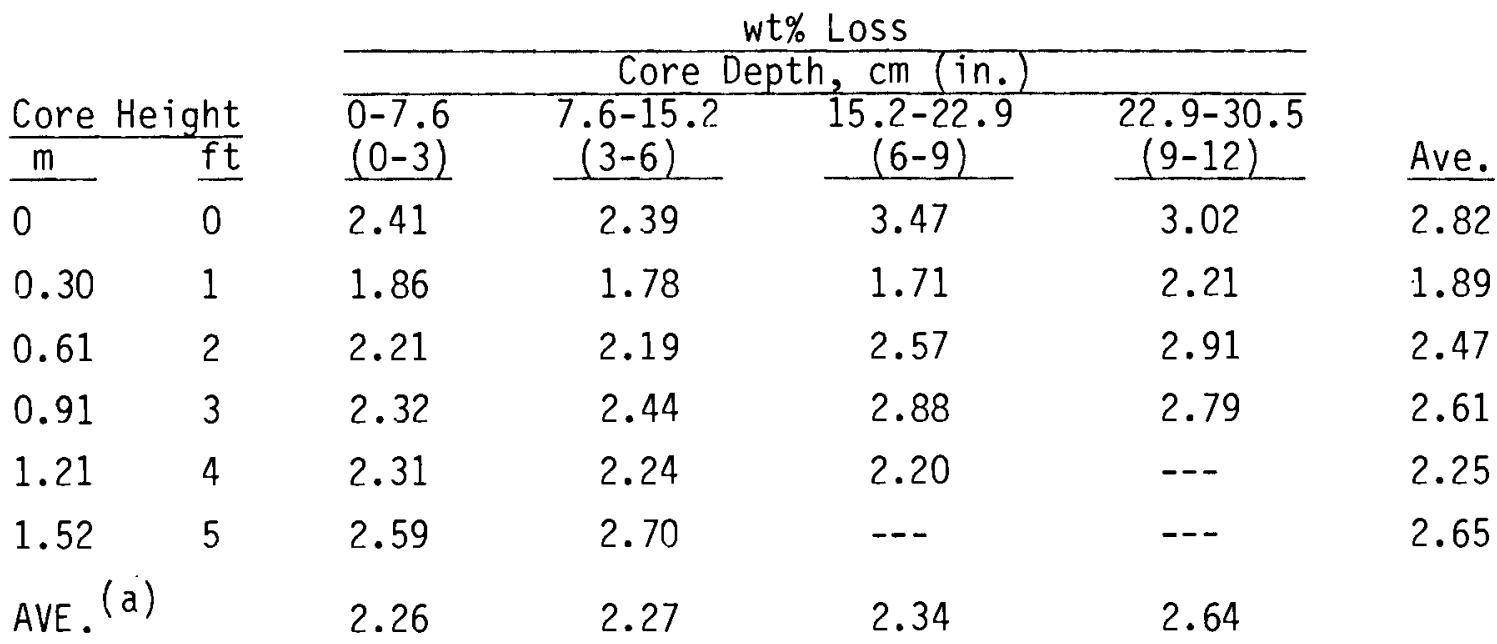

Mean Soxhlet Weight Loss $(a) 2.37 \% \pm 0.13 \%$

(a) Data from lower $0.30-\mathrm{m}(1-\mathrm{ft})$ level omitted from calculated averages.

to take advantage of higher melting temperature. After $5 \mathrm{~h}$ of operation, the frit feeder electronics malfunctioned, adding less frit than desired. This condition was discovered only after completing the run. 
After cooling, both canisters were cut open and samples were core-drilled. The glass in canister 174 was shiny black with a dull-mat textured zone at the bottom. Canister 172 contained a duller appearing glass. Both contained a number of 6 - to 16 -mm-dia metallic buttons at the bottom of the canister. The buttons shown in Figure 4 contained 70 to 75 wt\% metal: $90 \%$ nickel with traces of iron, lead and zinc. A white crystalline coating was found on the exposed canister walls and exposed glass surfaces of both canisters. This coating contained 50 to $89 \%$ water soluble salts, mainly sodium chloride with the remainder a mixture of frit and calcine oxides. The normalized chemical analysis for canisters 174 and 172 glass samples are presented in Tables 15 and 16 . Summaries of this data in Tables 17 and 18 show that the glass in the bottom of both canisters contained 42 to $45 \mathrm{wt} \%$ calcine. The microstructures of these bottom samples were more homogeneous than in canister 177, containing larger spinel crystals generally surrounded by acmite crystals (Figures 5 and 6 ). The bulk glass in both canisters again contained less than the defined 28 wt\% calcine. However, greater compositional variation was found in canister 174

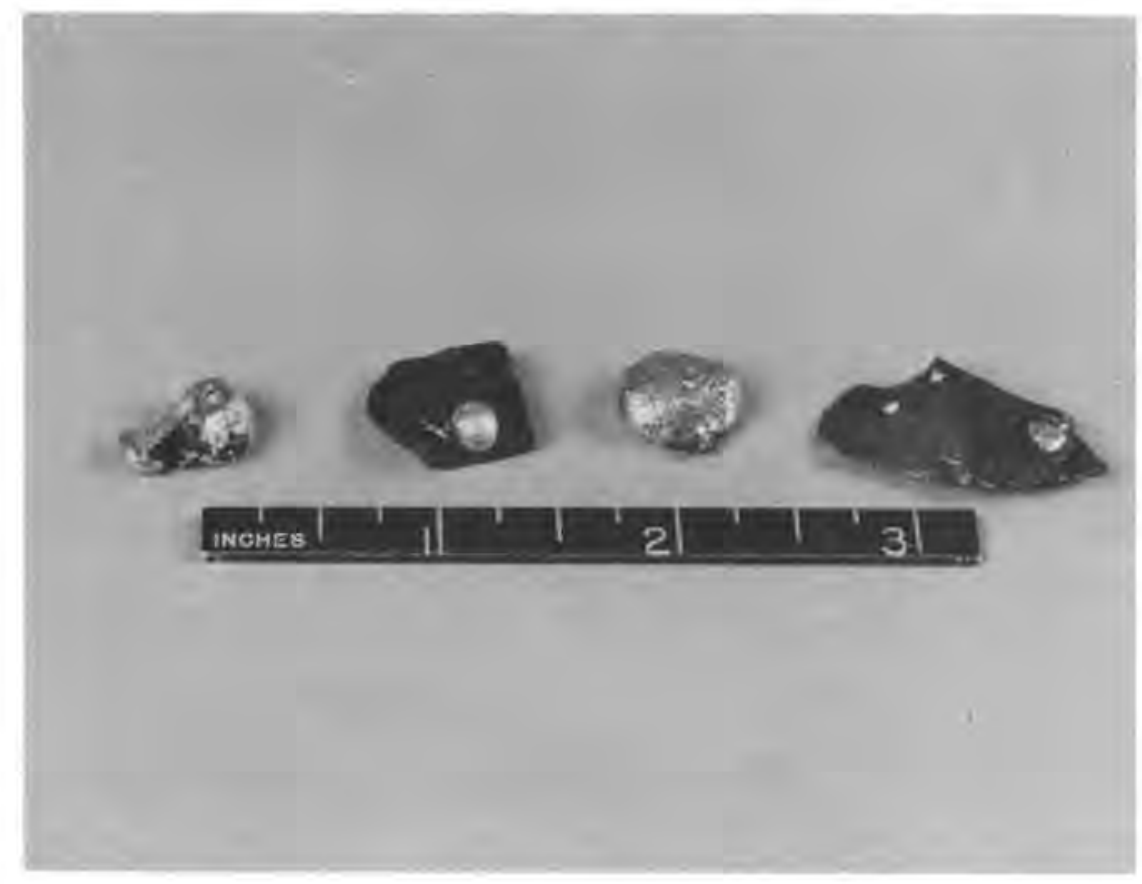

FIGURE 4. Metallic Buttons Removed from Bottom of Canisters 172 and 174 
TABLE 15. Normalized Composition of Canister 174 Samples

\begin{tabular}{|c|c|c|c|c|c|c|c|c|c|c|c|c|}
\hline \multicolumn{2}{|c|}{$\begin{array}{c}\text { Core } \\
\text { Height } \\
\end{array}$} & \multicolumn{2}{|c|}{ Core Depth } & \multicolumn{9}{|c|}{ Oxide wt\% } \\
\hline$m$ & $\overline{f t}$ & $\mathrm{~cm}$ & in. & $\overline{\mathrm{Si}_{0}} 2$ & $\mathrm{Al}_{2} \mathrm{O}_{3}$ & $\underline{B}_{2} \underline{0}_{3}$ & $\mathrm{Ca0}$ & $\mathrm{Fe}_{2}{ }_{-}{ }_{3}$ & $\underline{L i}_{2} \underline{O}$ & $\mathrm{Na}_{2} \underline{0}$ & $\mathrm{MnO}_{2}$ & $\mathrm{NiO}$ \\
\hline \multicolumn{2}{|c|}{ Defined } & & & 44.0 & 3.7 & 8.0 & 5.1 & 14.7 & 3.2 & 15.5 & 3.9 & 1.7 \\
\hline 0 & 0 & $\begin{array}{l}0-10.2 \\
10.2-20.3 \\
20.3-30.5\end{array}$ & $\begin{array}{l}0-4 \\
4-8 \\
8-12\end{array}$ & $\begin{array}{l}40.4 \\
41.9 \\
35.5\end{array}$ & $\begin{array}{l}5.8 \\
5.7 \\
6.4\end{array}$ & $\begin{array}{l}6.6 \\
6.8 \\
5.7\end{array}$ & $\begin{array}{l}5.6 \\
5.4 \\
5.2\end{array}$ & $\begin{array}{l}16.2 \\
15.0 \\
20.8\end{array}$ & $\begin{array}{l}2.6 \\
2.9 \\
2.3\end{array}$ & $\begin{array}{l}15.8 \\
16.8 \\
15.5\end{array}$ & $\begin{array}{l}3.9 \\
3.0 \\
4.8\end{array}$ & $\begin{array}{l}2.5 \\
2.0 \\
3.3\end{array}$ \\
\hline 0.30 & 1 & $\begin{array}{l}0-10.2 \\
10.2-20.3 \\
20.3-30.5\end{array}$ & $\begin{array}{l}0-4 \\
4-8 \\
8-12\end{array}$ & $\begin{array}{l}48.6 \\
46.2 \\
43.1\end{array}$ & $\begin{array}{l}3.8 \\
4.2 \\
5.8\end{array}$ & $\begin{array}{l}8.2 \\
7.7 \\
7.2\end{array}$ & $\begin{array}{l}5.2 \\
5.3 \\
5.8\end{array}$ & $\begin{array}{l}11.1 \\
12.0 \\
15.9\end{array}$ & $\begin{array}{l}3.4 \\
3.2 \\
2.8\end{array}$ & $\begin{array}{l}13.5 \\
16.0 \\
14.0\end{array}$ & $\begin{array}{l}4.2 \\
3.3 \\
2.7\end{array}$ & $\begin{array}{l}1.7 \\
1.6 \\
2.2\end{array}$ \\
\hline 0.61 & 2 & $\begin{array}{l}0-10.2 \\
10.2-20.3 \\
20.3-30.5\end{array}$ & $\begin{array}{l}0-4 \\
4-8 \\
8-12\end{array}$ & $\begin{array}{l}48.5 \\
46.8 \\
46.1\end{array}$ & $\begin{array}{l}3.6 \\
3.6 \\
3.8\end{array}$ & $\begin{array}{l}8.2 \\
7.9 \\
7.7\end{array}$ & $\begin{array}{l}5.3 \\
5.0 \\
5.1\end{array}$ & $\begin{array}{l}10.8 \\
10.5 \\
11.3\end{array}$ & $\begin{array}{l}3.0 \\
3.3 \\
3.3\end{array}$ & $\begin{array}{l}14.3 \\
17.0 \\
17.3\end{array}$ & $\begin{array}{l}4.2 \\
4.0 \\
3.4\end{array}$ & $\begin{array}{l}1.6 \\
1.6 \\
1.5\end{array}$ \\
\hline 0.91 & 3 & $\begin{array}{l}0-10.2 \\
10.2-20.3 \\
20.3-30.5\end{array}$ & $\begin{array}{l}0-4 \\
4-8 \\
8-12\end{array}$ & $\begin{array}{l}46.6 \\
46.4 \\
44.5\end{array}$ & $\begin{array}{l}3.5 \\
3.6 \\
4.3\end{array}$ & $\begin{array}{l}7.8 \\
7.8 \\
7.4\end{array}$ & $\begin{array}{l}5.0 \\
5.0 \\
5.4\end{array}$ & $\begin{array}{l}10.5 \\
10.9 \\
13.4\end{array}$ & $\begin{array}{l}3.3 \\
3.3 \\
3.2\end{array}$ & $\begin{array}{l}17.3 \\
17.0 \\
16.5\end{array}$ & $\begin{array}{l}4.0 \\
3.8 \\
2.9\end{array}$ & $\begin{array}{l}1.6 \\
1.6 \\
2.1\end{array}$ \\
\hline 1.21 & 4 & $\begin{array}{l}0-10.2 \\
10.2-20.3 \\
20.3-30.5\end{array}$ & $\begin{array}{l}0-4 \\
4-8 \\
8-12\end{array}$ & $\begin{array}{l}47.7 \\
46.3 \\
43.3\end{array}$ & $\begin{array}{l}3.6 \\
3.7 \\
4.3\end{array}$ & $\begin{array}{l}8.0 \\
7.9 \\
7.2\end{array}$ & $\begin{array}{l}5.2 \\
5.1 \\
5.2\end{array}$ & $\begin{array}{l}10.6 \\
11.0 \\
13.1\end{array}$ & $\begin{array}{l}3.1 \\
3.4 \\
3.0\end{array}$ & $\begin{array}{l}15.8 \\
17.1 \\
18.6\end{array}$ & $\begin{array}{l}4.1 \\
3.7 \\
3.0\end{array}$ & $\begin{array}{l}1.6 \\
1.5 \\
2.0\end{array}$ \\
\hline 1.52 & 5 & $0-10.2$ & $0-4$ & 47.0 & 3.5 & 8.1 & 5.2 & 11.1 & 3.0 & 15.7 & 4.2 & 1.7 \\
\hline
\end{tabular}

TABLE 16. Normalized Composition of Canister 172 Samples

\begin{tabular}{|c|c|c|c|c|c|c|c|c|c|c|c|c|}
\hline \multicolumn{2}{|c|}{$\begin{array}{c}\text { Core } \\
\text { Height }\end{array}$} & \multicolumn{2}{|c|}{ Core Depth } & \multicolumn{9}{|c|}{ Oxide wt\% } \\
\hline $\mathrm{m}$ & $\underline{\mathrm{ft}}$ & $\mathrm{cm}$ & in. & $\mathrm{SiO}_{2}$ & $\mathrm{AI}_{2}-_{3}$ & $\mathrm{~B}_{2}-\underline{0}_{3}$ & $\mathrm{CaO}$ & $\mathrm{Fe}_{2} \underline{0}_{3}$ & $\underline{L}{ }^{i} 2-0$ & $\underline{\mathrm{Na}}_{2} \mathrm{O}$ & $\mathrm{MnO}_{2}$ & $\underline{\mathrm{NiO}}$ \\
\hline Defir & & & & 44.0 & 3.7 & 8.0 & 5.1 & 14.7 & 3.2 & 15.5 & 3.9 & 1.7 \\
\hline 0 & 0 & $\begin{array}{l}0-10.2 \\
10.2-20.3 \\
20.3-30.5\end{array}$ & $\begin{array}{l}0-4 \\
4-8 \\
8-12\end{array}$ & $\begin{array}{l}39.4 \\
39.7 \\
36.2\end{array}$ & $\begin{array}{l}5.9 \\
5.9 \\
6.3\end{array}$ & $\begin{array}{l}6.5 \\
6.5 \\
5.9\end{array}$ & $\begin{array}{l}5.5 \\
5.4 \\
5.1\end{array}$ & $\begin{array}{l}18.2 \\
18.0 \\
22.7\end{array}$ & $\begin{array}{l}2.5 \\
2.5 \\
2.3\end{array}$ & $\begin{array}{l}15.0 \\
14.9 \\
13.4\end{array}$ & $\begin{array}{l}4.9 \\
4.9 \\
5.0\end{array}$ & $\begin{array}{l}1.8 \\
1.7 \\
2.8\end{array}$ \\
\hline 0.30 & 1 & $\begin{array}{l}0-10.2 \\
10.2-20.3 \\
20.3-30.5\end{array}$ & $\begin{array}{l}0-4 \\
4-8 \\
8-12\end{array}$ & $\begin{array}{l}43.6 \\
42.1 \\
41.8\end{array}$ & $\begin{array}{l}4.4 \\
4.7 \\
4.9\end{array}$ & $\begin{array}{l}7.3 \\
6.8 \\
6.8\end{array}$ & $\begin{array}{l}4.9 \\
5.7 \\
5.5\end{array}$ & $\begin{array}{l}15.9 \\
16.8 \\
17.5\end{array}$ & $\begin{array}{l}3.0 \\
2.6 \\
2.6\end{array}$ & $\begin{array}{l}15.1 \\
15.0 \\
14.2\end{array}$ & $\begin{array}{l}3.6 \\
4.0 \\
4.1\end{array}$ & $\begin{array}{l}1.7 \\
2.0 \\
2.1\end{array}$ \\
\hline 0.61 & 2 & $\begin{array}{l}0-10.2 \\
10.2-20.3 \\
20.3-30.5\end{array}$ & $\begin{array}{l}0-4 \\
4-8 \\
8-12\end{array}$ & $\begin{array}{l}44.3 \\
44.6 \\
43.0\end{array}$ & $\begin{array}{l}4.4 \\
4.3 \\
4.3\end{array}$ & $\begin{array}{l}7.4 \\
7.3 \\
6.8\end{array}$ & $\begin{array}{l}5.2 \\
5.2 \\
5.7\end{array}$ & $\begin{array}{l}16.2 \\
15.6 \\
17.0\end{array}$ & $\begin{array}{l}2.8 \\
2.9 \\
2.6\end{array}$ & $\begin{array}{l}13.9 \\
14.3 \\
14.3\end{array}$ & $\begin{array}{l}3.8 \\
3.7 \\
3.7\end{array}$ & $\begin{array}{l}1.6 \\
1.5 \\
2.2\end{array}$ \\
\hline 0.91 & 3 & $\begin{array}{l}0-10.2 \\
10.2-20.3\end{array}$ & $\begin{array}{l}0-4 \\
4-8\end{array}$ & $\begin{array}{l}44.3 \\
43.2\end{array}$ & $\begin{array}{l}4.5 \\
4.2\end{array}$ & $\begin{array}{l}7.4 \\
7.0\end{array}$ & $\begin{array}{l}5.3 \\
5.6\end{array}$ & $\begin{array}{l}16.0 \\
16.5\end{array}$ & $\begin{array}{l}2.7 \\
2.7\end{array}$ & $\begin{array}{l}13.9 \\
14.6\end{array}$ & $\begin{array}{l}3.8 \\
3.6\end{array}$ & $\begin{array}{l}1.6 \\
2.1\end{array}$ \\
\hline 1.21 & 4 & $\begin{array}{l}0-10.2 \\
10.2-20.3\end{array}$ & $\begin{array}{l}0-4 \\
4-8\end{array}$ & $\begin{array}{l}43.9 \\
43.2\end{array}$ & $\begin{array}{l}4.5 \\
4.4\end{array}$ & $\begin{array}{l}7.4 \\
7.2\end{array}$ & $\begin{array}{l}5.4 \\
5.5\end{array}$ & $\begin{array}{l}16.6 \\
16.8\end{array}$ & $\begin{array}{l}3.0 \\
2.7\end{array}$ & $\begin{array}{l}13.1 \\
14.3\end{array}$ & $\begin{array}{l}3.7 \\
3.4\end{array}$ & $\begin{array}{l}2.0 \\
2.0\end{array}$ \\
\hline
\end{tabular}


TABLE 17. Average Composition of Canister 174 Samples

\begin{tabular}{|c|c|c|c|c|c|c|c|c|c|c|}
\hline \multicolumn{2}{|c|}{ Core Height } & \multicolumn{9}{|c|}{ Oxide wt\% } \\
\hline $\mathrm{m}$ & in. & $\overline{\mathrm{SiO}_{2}}$ & $\mathrm{Al}_{2} \underline{O}_{3}$ & $\mathrm{~B}_{2} \mathrm{O}_{-3}$ & $\mathrm{CaO}$ & $\mathrm{Fe}_{2} \mathrm{O}_{3}$ & $\underline{L i}_{2} 2-$ & $\underline{\mathrm{Na}}_{2} \underline{0}$ & $\mathrm{MnO}_{2}$ & $\underline{\mathrm{NiO}}$ \\
\hline Defined & & $44 . \overline{0}$ & 3.7 & 8.0 & 5.1 & 14.7 & 3.2 & 15.5 & 3.9 & 1.7 \\
\hline 0 & 0 & 39.3 & 6.0 & 6.4 & 5.4 & 17.3 & 2.6 & 16.0 & 3.9 & 2.6 \\
\hline 0.30 & 1 & 46.0 & 4.6 & 7.7 & 5.4 & 13.0 & 3.1 & 14.5 & 3.4 & 1.8 \\
\hline 0.61 & 2 & 47.1 & 3.7 & 7.9 & 5.1 & 10.9 & 3.2 & 16.2 & 3.9 & 1.6 \\
\hline 0.91 & 3 & 45.8 & 3.8 & 7.7 & 5.1 & 11.6 & 3.3 & 16.9 & 3.6 & 1.8 \\
\hline 1.21 & 4 & 45.8 & 3.9 & 7.7 & 5.2 & 11.6 & 3.2 & 17.2 & 3.6 & 1.7 \\
\hline 1.52 & 5 & 47.0 & 3.5 & 8.1 & 5.2 & 11.1 & 3.0 & 15.7 & 4.2 & 1.7 \\
\hline
\end{tabular}

\begin{tabular}{|c|c|c|c|c|c|c|c|c|c|c|}
\hline \multicolumn{2}{|c|}{ Core $\operatorname{Depth}^{(a)}$} & & & & & & & & & \\
\hline $\mathrm{cm}$ & in. & & & & & & & & & \\
\hline $0-10.2$ & $0-4$ & 47.7 & 3.6 & 8.1 & 5.2 & 10.8 & 3.2 & 15.3 & 4.1 & 1.6 \\
\hline $10.2-20.3$ & $4-8$ & 46.4 & 3.8 & 7.8 & 5.1 & 11.1 & 3.3 & 16.8 & 3.7 & 1.6 \\
\hline $20.3-30.5$ & $8-12$ & 44.2 & 4.6 & 7.4 & 5.4 & 13.4 & 3.1 & 16.6 & 3.0 & 2.0 \\
\hline $\begin{array}{l}\text { AVE. (a) } \\
\text { STD. DEV. }\end{array}$ & & $\begin{array}{r}46.2 \\
1.7\end{array}$ & $\begin{array}{l}3.9 \\
0.6\end{array}$ & $\begin{array}{l}7.8 \\
0.3\end{array}$ & $\begin{array}{l}5.2 \\
0.2\end{array}$ & $\begin{array}{r}11.7 \\
1.6\end{array}$ & $\begin{array}{l}3.2 \\
0.2\end{array}$ & $\begin{array}{r}16.2 \\
1.5\end{array}$ & $\begin{array}{l}3.6 \\
0.5\end{array}$ & $\begin{array}{l}1.7 \\
0.2\end{array}$ \\
\hline
\end{tabular}

(a) Data from lower $0.30 \mathrm{~m}(1 \mathrm{ft}$ ) omitted from calculated averages.

samples. In general, the calcine-rich region on the canister bottom contained 5 to $10 \mathrm{wt} \%$ spinel compared to 0 to $15 \mathrm{wt} \%$ acmite in the rest of either canister (Tables 19 and 20). The microstructure of the acmite-containing zones in canister 174 and 172 are shown in Figures 7 through 10. Although spinel was not identified in these samples by $x$-ray diffraction, small crystallines are seen associated with many acmite crystals. The glass in canister 174 contained less calcine and was processed at lower temperatures than the glass in canister 172. This resulted in smaller spinel and acmite crystals in canister 174 . On the other hand, canister 172, besides containing larger crystals, displayed considerable porosity and more microcracking. This accounts for the du11 appearance of the glass noted when canister 172 was opened. 
TABLE 18. Average Composition of Canister 172 Samples

Core Height

$\frac{\mathrm{m}}{\mathrm{ft}}$

Defined

0
0.30
0.61
0.91
1.21

$\mathrm{ft}$

$$
\text { Sin }
$$

$\overline{\mathrm{SiO}}_{2} \quad \underline{\mathrm{Al}}_{2} \underline{-}_{3} \quad \underline{\mathrm{B}}_{2} \underline{-}_{3} \quad \underline{\mathrm{CaO}}_{3} \quad \underline{\mathrm{Fe}}_{2}{ }_{-}{ }_{3}$

$\begin{array}{lllll}44.0 & 3.7 & 8.0 & 5.1 & 14.7\end{array}$

38.4

6.0

6.3

5.319 .6

$4.7 \quad 7.0$

$5.4 \quad 16.7$

$4.3 \quad 7.2$

$5.4 \quad 16.3$

$4.4 \quad 7.2$

5.416 .2

$4.4 \quad 7.3$
43.8

43.6
5.416 .7

$\frac{\mathrm{Li}}{3.2} 2^{0}$

$\underline{N a}_{2}$ O

$\frac{\mathrm{Na}_{2}}{15.5} \quad \frac{\mathrm{MnO}}{3.9} \quad \frac{\mathrm{NiO}}{1.7}$

$2.4 \quad 14.4 \quad 4.9 \quad 2.1$

$\begin{array}{llll}2.7 & 14.8 & 3.9 & 1.9\end{array}$

$\begin{array}{llll}2.8 & 14.2 & 3.7 & 1.8\end{array}$

$\begin{array}{llll}2.7 & 14.2 & 3.7 & 1.8\end{array}$

$\begin{array}{llll}2.8 & 13.7 & 3.6 & 2.0\end{array}$

\section{Core Depth}

$\frac{\mathrm{cm}}{0-10.2} \frac{\text { in. }}{0-4}$

$10.2-20.3 \quad 4-8$

44.0

$4.4 \quad 7.4$

$5.2 \quad 16.2$

$2.9 \quad 14.0$

3.7

1.7

$20.3-30.5 \quad 8-12$

43.2

$4.4 \quad 7.1$

$5.5 \quad 16.4$

$2.7 \quad 14.6$

$3.7 \quad 1.9$

AVE. (a)

STD. DEV.

42.4

4.6

$\begin{array}{lll}6.8 & 5.6 \quad 17.2\end{array}$

$\begin{array}{llll}2.6 & 14.2 & 3.9 & 2.2\end{array}$

$\begin{array}{lllll}43.4 & 4.5 & 7.1 & 5.4 & 16.5\end{array}$

$\begin{array}{rrrrr}0.9 & 0.2 & 0.3 & 0.2 & 0.6\end{array}$

2.8

14.3

0.6

$3.7 \quad 1.9$

0.20 .2

(a) Data from lower $0.30 \mathrm{~m}(1 \mathrm{ft})$ omitted from calculated averages.

Chemical durability did not appear to coincide with composition, microstructure or crystal content variation. The average soxhlet weight loss values were 2.41 and 3.40 wt\% for canisters 174 and 172 while pH4 weight loss values for both were approximately $84.7 \mathrm{wt} \%$ loss $\pm 0.5 \mathrm{wt} \%$. This data is presented in Tables 21 through 24. The presence of the spinel phase on the canister floor again did not alter the product durability.

FS-ICM-28 AND FS-ICM-29 (AUGUST 1980)

The object of these melting trials was to determine the effect that additional mixing would have on product quality. FS-ICM-28 was carried out in a typical manner except that the melt was continuously mixed by an air sparge. Canister 180 was filled with $1164 \mathrm{~kg}$ of glass at $85 \mathrm{~kg} / \mathrm{h}\left(324 \mathrm{~kg} / \mathrm{h} / \mathrm{m}^{2}\right)$. In the second trial, FS-ICM-29, the spray calciner was operated at lower barrel 

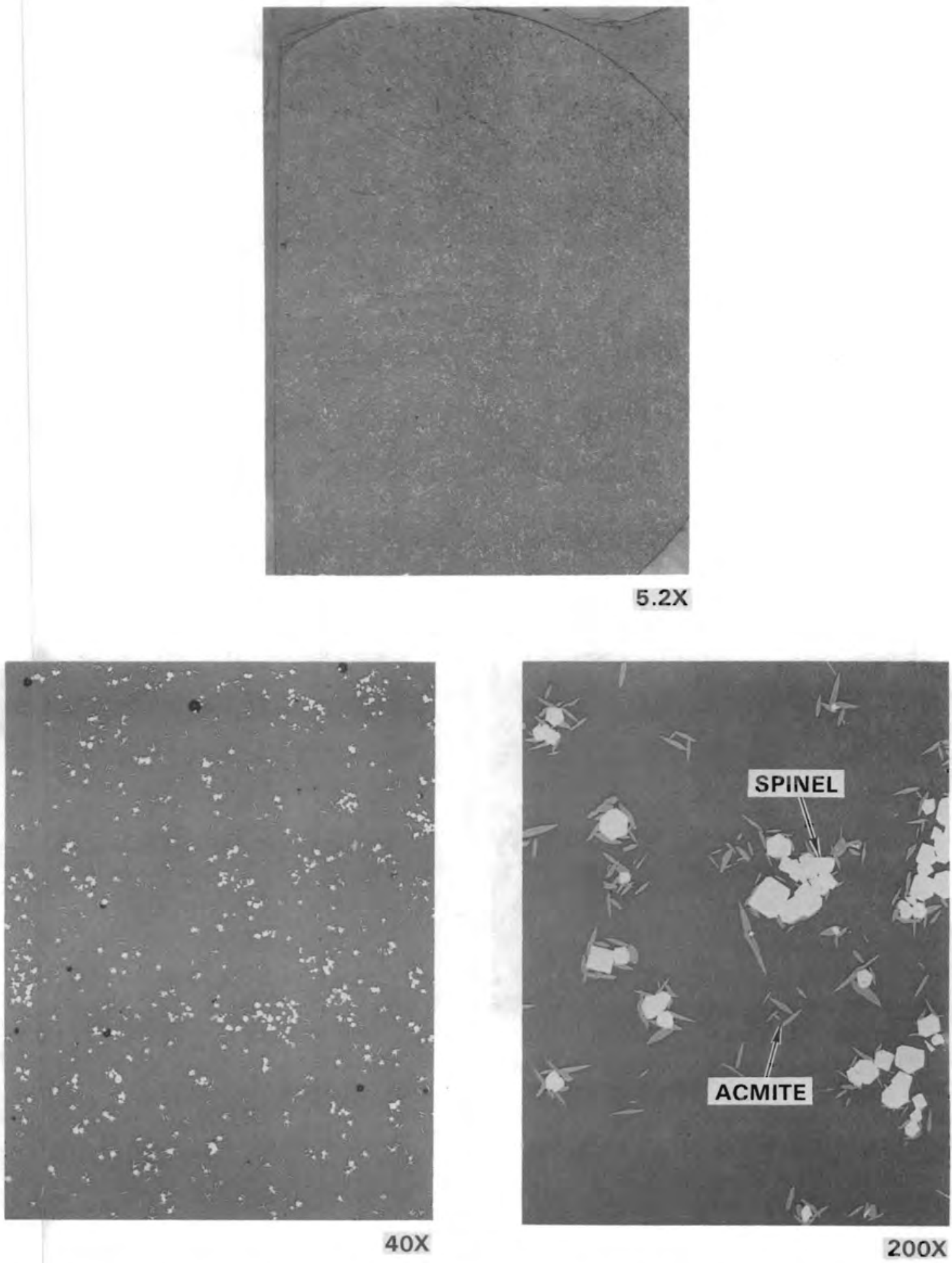

FIGURE 5. Microstructure of Canister 174 Sample [Height $0 \mathrm{~m}(0 \mathrm{ft})$; Depth 20.3-30.5 cm (8-12 in.)] 

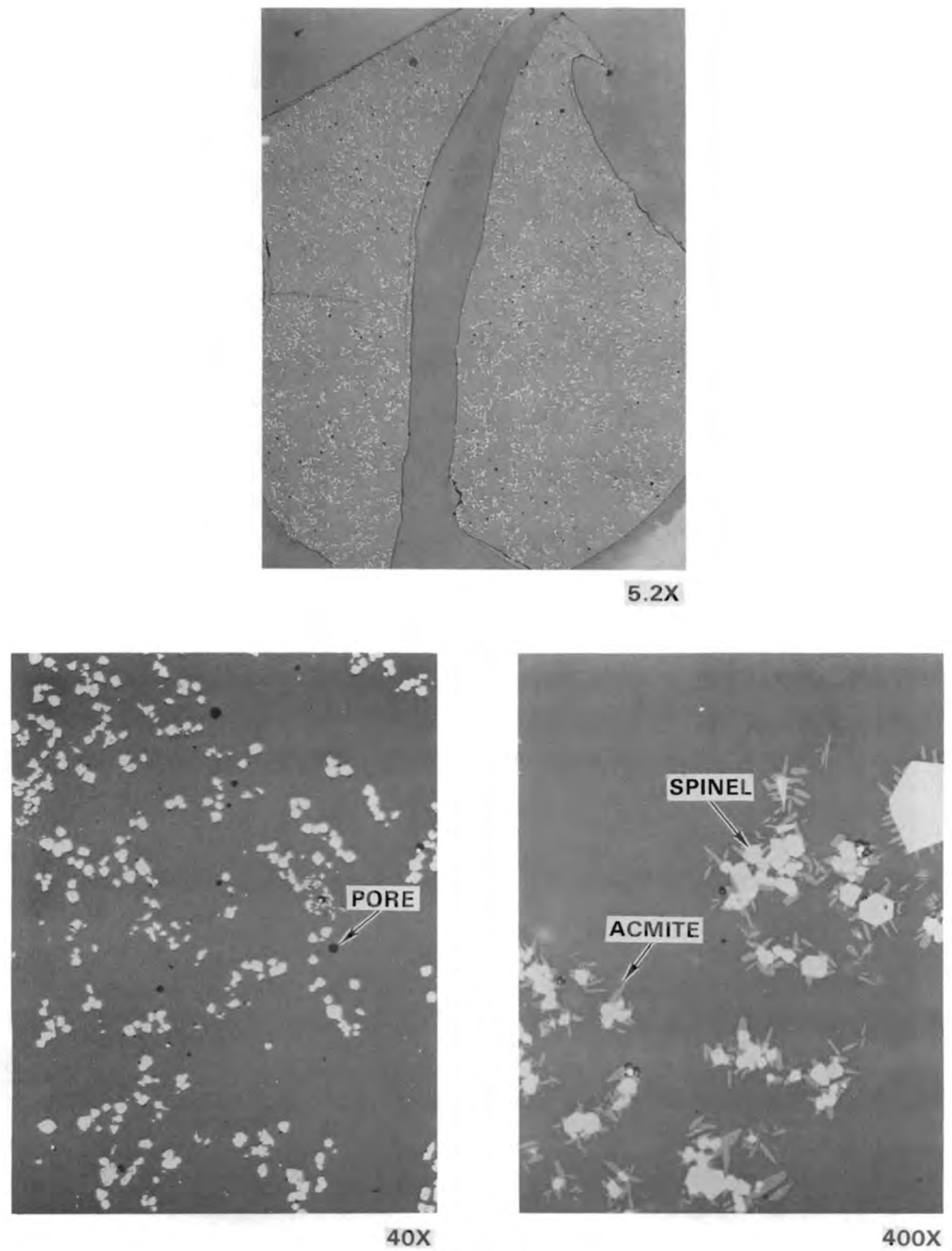

FIGURE 6. Microstructure of Canister 172 Samples [Height $0 \mathrm{~m}(0 \mathrm{ft})$; Depth 20.3-30.5 cm (8-12 in.)] 
TABLE 19. Crystalline Content of Canister 174 Samples

\begin{tabular}{|c|c|c|c|c|}
\hline \multicolumn{2}{|c|}{ Core Height } & \multicolumn{3}{|c|}{ Core Depth, cm (in.) } \\
\hline$\frac{\text { Core }}{\mathrm{m}}$ & $\frac{g h t}{f t}$ & $\begin{array}{l}0-10.2 \\
(0-4)^{2}\end{array}$ & $\begin{array}{c}10.2-20.3 \\
(4-8) \\
\end{array}$ & $\begin{array}{c}20.3-30.5 \\
(8-12)\end{array}$ \\
\hline 0 & 0 & $5 \%$ Spine ${ }^{(a)}$ & $\begin{array}{r}5 \% \text { Spinel } \\
<5 \% \text { Acmite }(b)\end{array}$ & 5-10\% Spine 1 \\
\hline 0.30 & 1 & 0 & $<5 \%$ Acmite & 5-10\% Spinel \\
\hline 0.61 & 2 & $<5 \%$ Acmite & $10 \%$ Acmite & 10-15\% Acmite \\
\hline 0.91 & 3 & $<5 \%$ Acmite & $5 \%$ Acmite & $5 \%$ Acmite \\
\hline 1.21 & 4 & 0 & 5-10\% Acmite & 0 \\
\hline 1.52 & 5 & 0 & --- & --- \\
\hline
\end{tabular}

(a) Spinel - $(\mathrm{Ni}, \mathrm{Mn}) \mathrm{Fe}_{2} \mathrm{O}_{4}$

(b) Acmite - $\mathrm{NaFe}\left(\mathrm{SiO}_{3}\right)_{2}$

TABLE 20. Crystalline Content of Canister 172 Samples

\begin{tabular}{|c|c|c|c|c|}
\hline \multicolumn{2}{|c|}{ Core Hejaht } & \multicolumn{3}{|c|}{ Core Depth, cm (in.) } \\
\hline$\frac{\text { Core }}{m}$ & $\frac{g h t}{f t}$ & $\begin{array}{r}0-10.2 \\
(0-4)\end{array}$ & $\begin{array}{c}10.2-20.3 \\
(4-8)\end{array}$ & $\begin{array}{c}20.3-30.5 \\
(8-12) \\
\end{array}$ \\
\hline 0 & $\overline{0}$ & $<2 \%$ Spinel $(a)$ & 0 & $5 \%$ Spinel \\
\hline 0.30 & 1 & $5 \%$ Acmite $^{(b)}$ & $<5 \%$ Acmite & $<5 \%$ Acmite \\
\hline 0.61 & 2 & $<5 \%$ Acmite & $<5 \%$ Acmite & $5 \%$ Acmite \\
\hline 0.91 & 3 & $<2 \%$ Acmite & $5 \%$ Acmite & \\
\hline & & 0 & 0 & \\
\hline
\end{tabular}

(a) Spinel - $(\mathrm{Ni}, \mathrm{Mn}) \mathrm{Fe}_{2} \mathrm{O}_{4}$

(b) Acmite - $\mathrm{NaFe}\left(\mathrm{SiO}_{3}\right)_{2}$

temperatures to allow the frit to be sprayed into the barrel along with the waste constituents. The low temperature $\left(400^{\circ} \mathrm{C}\right.$ instead of 700 to $\left.800^{\circ} \mathrm{C}\right)$ prevented the frit from melting and sticking to the calciner barrel, and also 1 imited the production rate to $20 \mathrm{~kg} / \mathrm{h}\left(76 \mathrm{~kg} / \mathrm{h} / \mathrm{m}^{2}\right)$. Canister 181 was filled with $805 \mathrm{~kg}$ of waste glass. Product samples have been taken; however, no analytical results are available. 


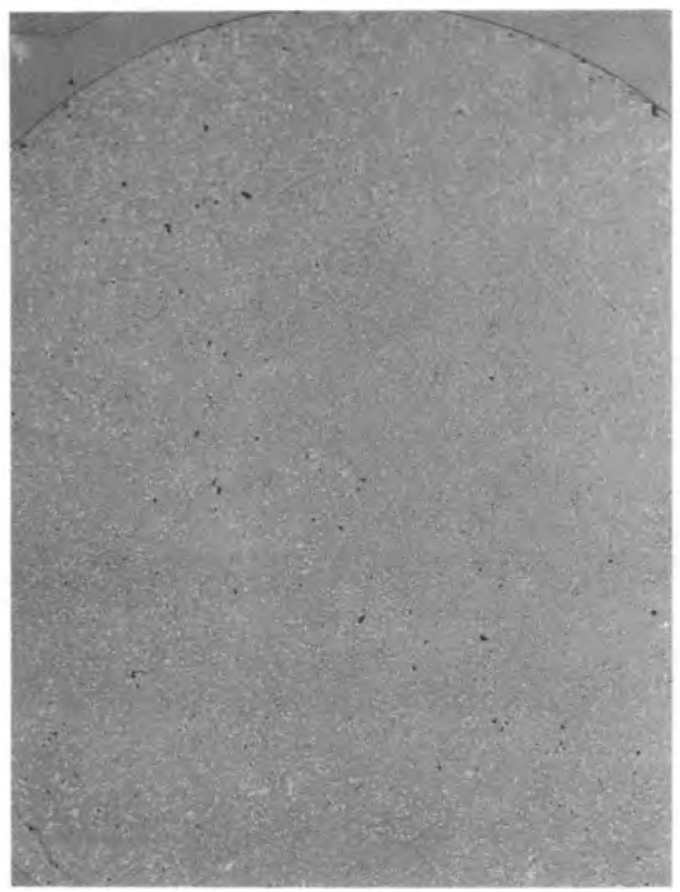

$5.2 X$
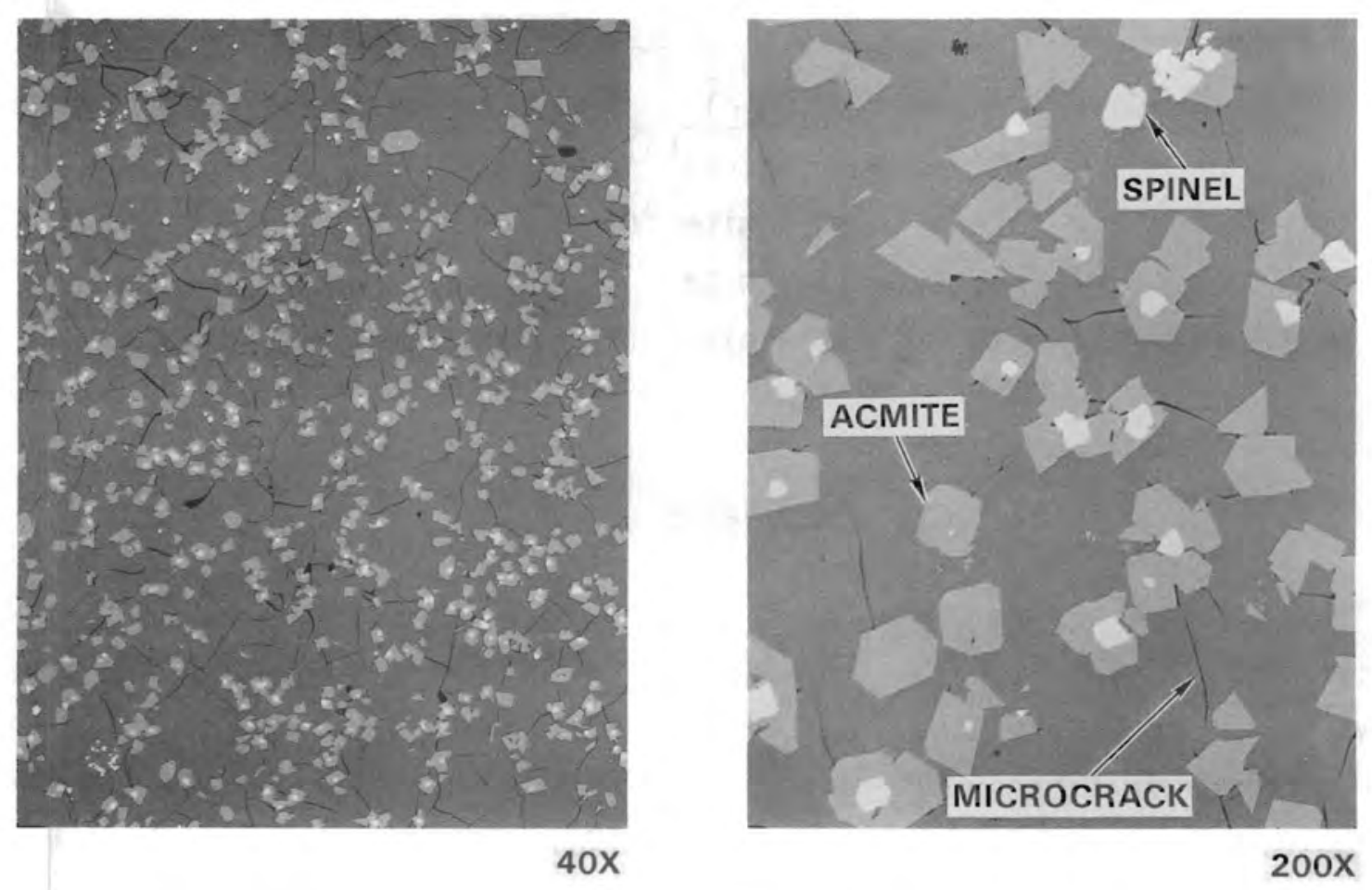

FIGURE 7. Microstructure of Canister 174 Sample [Height $0.61 \mathrm{~m}(2 \mathrm{ft})$; Depth 20.3-30.5 cm (8-12 in.)] 

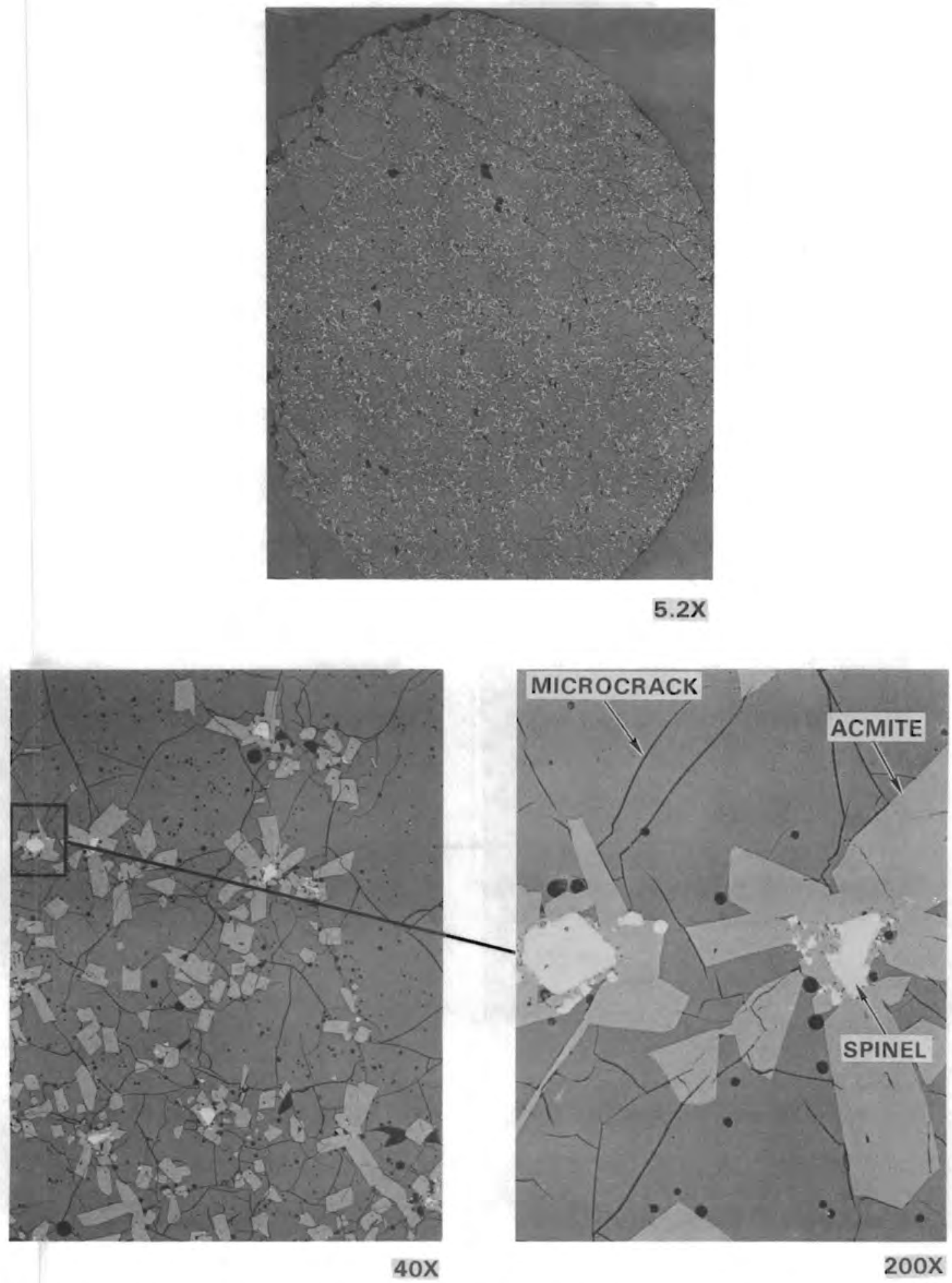

FIGURE 8. Microstructure of Canister 172 Sample [Height $0.61 \mathrm{~m}(2 \mathrm{ft})$; Depth 20.3-30.5 cm (8-12 in.)] 


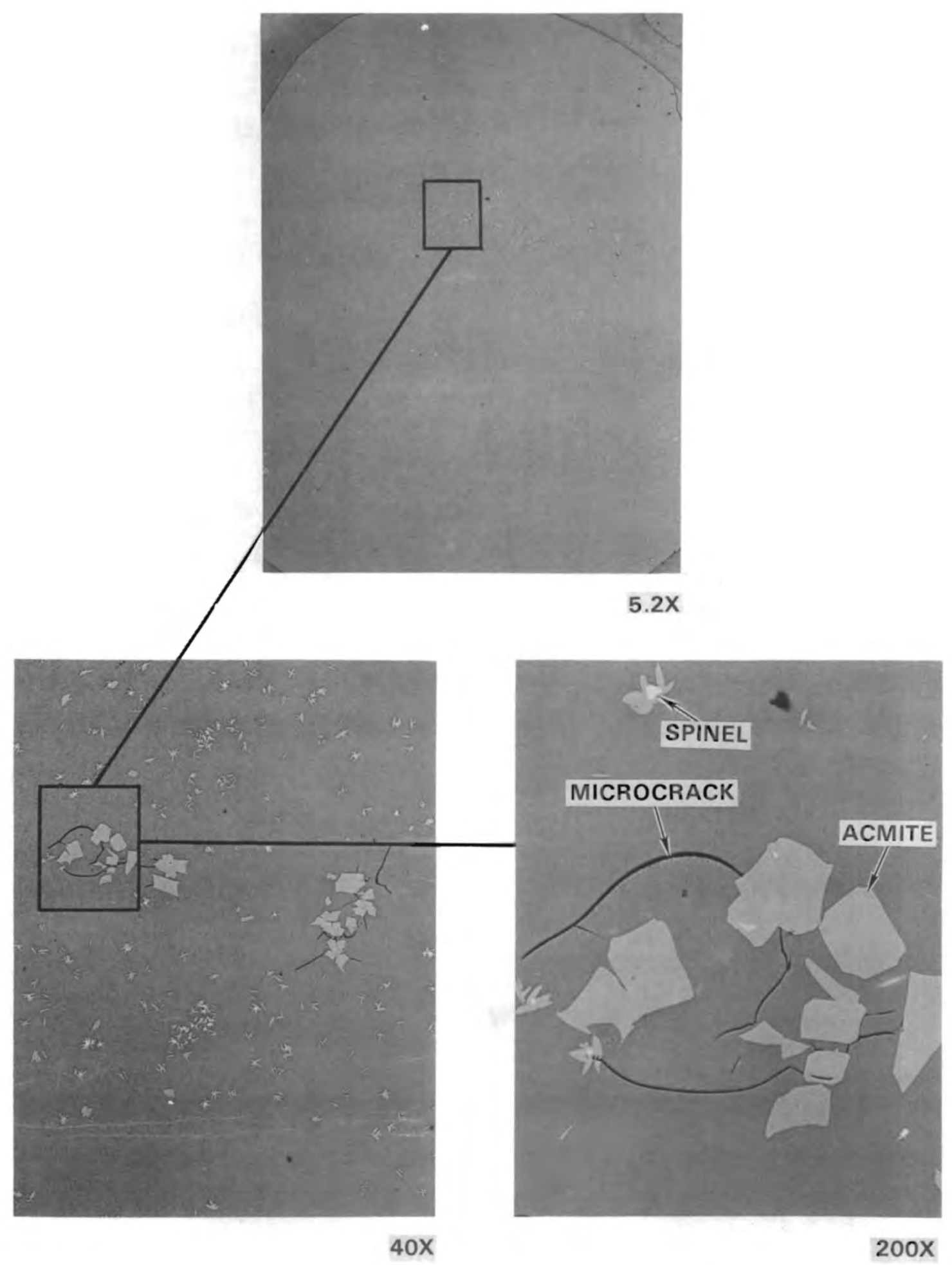

FIGURE 9. Microstructure of Canister 174 Sample [Height $1.21 \mathrm{~m}(4 \mathrm{ft})$; Depth 0-10.2 cm (0-4 in.)] 


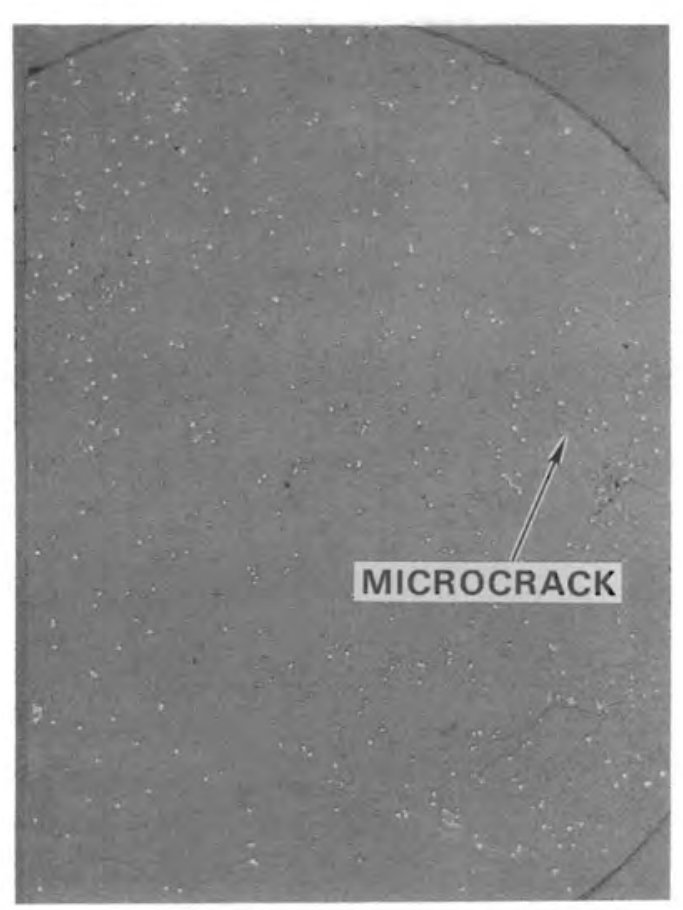

$5.2 X$

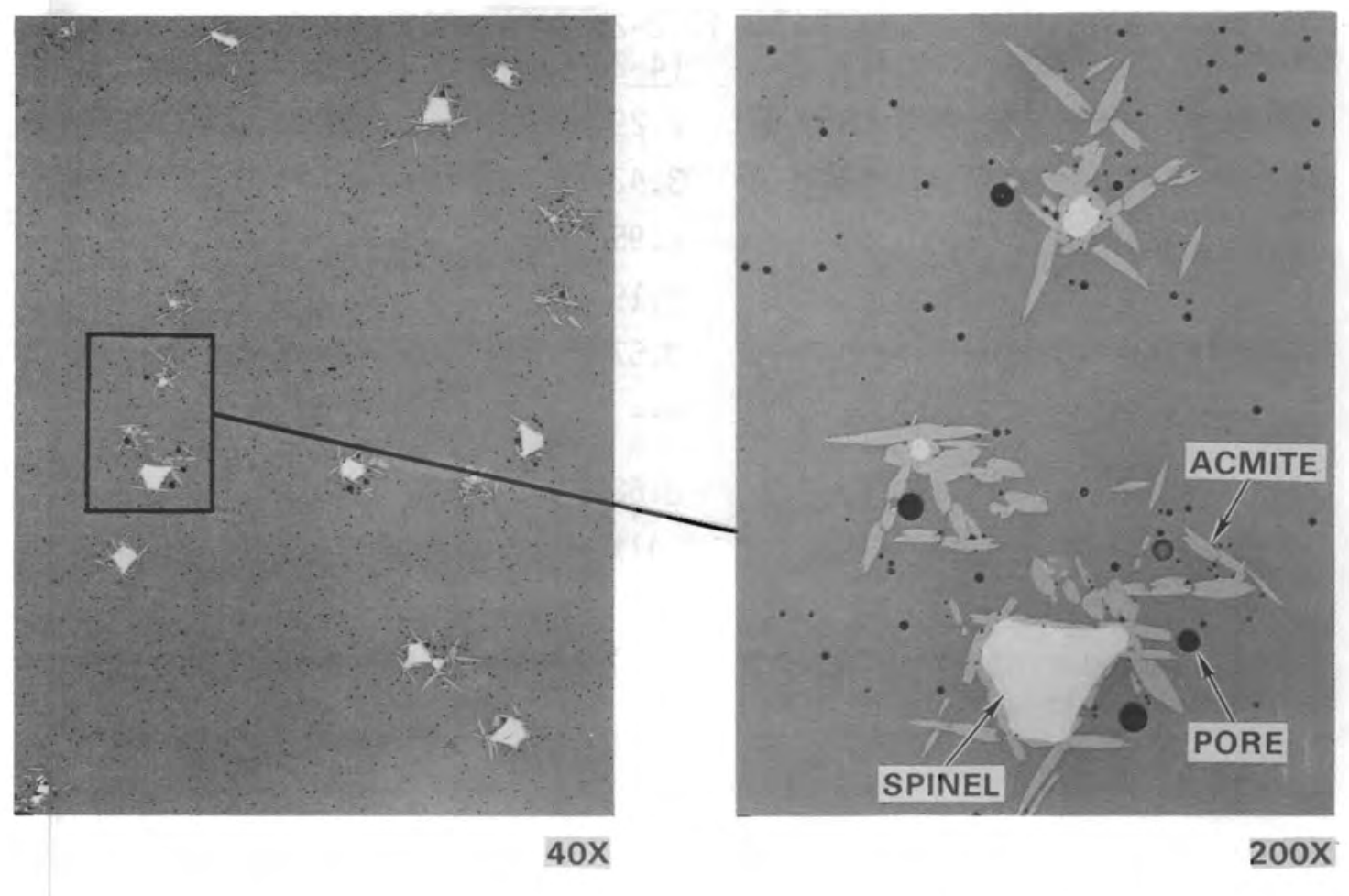

FIGURE_10. Microstructure of Canister 172 Sample [Height $1.21 \mathrm{~m}(4 \mathrm{ft})$; Depth 0-10.2 cm (0-4 in.)] 
TABLE 21. Weight Loss of Canister 174 Samples Under pH4 Conditions

\begin{tabular}{|c|c|c|c|c|c|}
\hline \multirow{3}{*}{\multicolumn{2}{|c|}{ Core Height }} & \multicolumn{3}{|c|}{ wt\% Loss } & \multirow{3}{*}{ Ave } \\
\hline & & \multirow{2}{*}{$\begin{array}{l}0-10 j^{2} \\
(0-4)^{2}\end{array}$} & \multirow{2}{*}{$\frac{e \text { Depth, cm }}{10.2-20.3}$} & \multirow{2}{*}{ 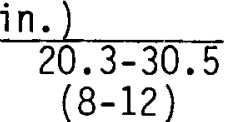 } & \\
\hline & & & & & \\
\hline 0 & 0 &.-- &.-- & --- & \\
\hline 0.30 & 1 & 91.3 & 78.5 & 91.4 & 87.1 \\
\hline 0.61 & 2 & 90.5 & 74.6 & 75.3 & 80.1 \\
\hline 0.91 & 3 & 85.9 & 82.0 & 82.2 & 83.4 \\
\hline 1.21 & 4 & 94.8 & 79.7 & --- & 87.2 \\
\hline AVE. & & 90.6 & 78.7 & 83.0 & \\
\hline
\end{tabular}

TABLE 22. Weight Loss of Canister 174 Samples Under Soxhlet Conditions

wt\% Loss

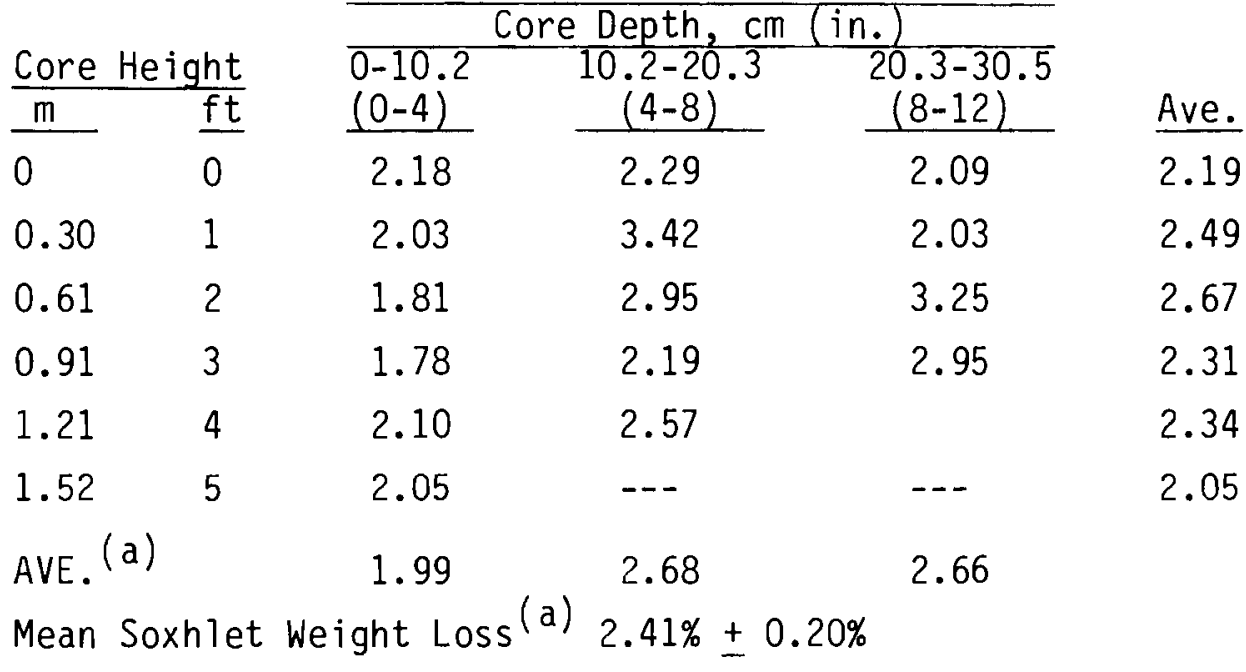

(a) Data from lower $0.30 \mathrm{~m}(1 \mathrm{ft})$ omitted from calculated averages. 
TABLE 23. Weight Loss of Canister 172 Samples Under pH4 Conditions

\begin{tabular}{|c|c|c|c|c|c|}
\hline \multirow{3}{*}{\multicolumn{2}{|c|}{ Core Height }} & \multicolumn{3}{|c|}{ wt\% Loss } & \multirow[b]{3}{*}{ Ave. } \\
\hline & & \multirow{2}{*}{$\begin{array}{l}0-10.2 \\
(0-4)^{2}\end{array}$} & \multicolumn{2}{|c|}{ ore Depth, cm (in.) } & \\
\hline & & & $\begin{array}{c}10.2-20.3 \\
(4-8) \\
\end{array}$ & $\begin{array}{c}20.3-30.5 \\
(8-12) \\
\end{array}$ & \\
\hline 0 & 0 & 88.5 & 91.7 & 87.0 & 89.0 \\
\hline 0.30 & 1 & 88.2 & 79.2 & 86.3 & 84.6 \\
\hline 0.61 & 2 & 92.6 & 77.1 & 76.8 & 82.2 \\
\hline 0.91 & 3 & 88.1 & 74.9 & -- & 81.5 \\
\hline 1.21 & 4 & 89.6 & 88.0 & --- & 88.8 \\
\hline AVE & & 89.6 & 79.8 & 81.6 & \\
\hline
\end{tabular}
(a) Data from lower $0.30 \mathrm{~m}(1 \mathrm{ft})$ omitted from calculated averages.

TABLE 24. Weight Loss of Canister 172 Samples Under Soxhlet Conditions wt\% Loss

\begin{tabular}{|c|c|c|c|c|c|}
\hline & \multicolumn{3}{|c|}{ Core Depth, $\mathrm{cm}$ (in.) } & \multirow[b]{3}{*}{ Ave. } \\
\hline & & $\overline{0-10.2}$ & $10.2-20.3$ & $20.3-30.5$ & \\
\hline \multicolumn{2}{|c|}{$\frac{\text { Core Height }}{\mathrm{m}} \frac{\mathrm{ft}}{\mathrm{f}}$} & $(0-4)$ & & $(8-12)$ & \\
\hline 0 & 0 & 3.32 & 2.91 & 1.90 & 2.71 \\
\hline 0.30 & 1 & 2.77 & 3.62 & 2.80 & 3.06 \\
\hline 0.61 & 2 & 2.33 & 5.82 & 4.39 & 4.18 \\
\hline 0.91 & 3 & 2.99 & 5.52 & --- & 4.26 \\
\hline 1.21 & 4 & 2.49 & 2.88 & --- & 2.68 \\
\hline \multicolumn{2}{|c|}{ AVE. (a) } & 2.78 & 4.15 & 3.03 & \\
\hline \multicolumn{5}{|c|}{ Mean Soxhlet Weight Loss $(\mathrm{a}) 3.40 \% \pm 0.61 \%$} & \\
\hline
\end{tabular}
(a) Data from lower $0.30 \mathrm{~m}(1 \mathrm{ft})$ omitted from calculated averages.





\section{LIQUID-FED CERAMIC MELTER}

From July 1979 to January 1980 three melting trials producing over $6000 \mathrm{~kg}$ of TDS-211 waste glass were run in the Liquid-Fed Ceramic Melter (LFCM). The LFCM is a joule-heated ceramic melter developed by PNL which converts a waste/ frit mixture into glass. An alternating current is passed between electrodes submerged in molten glass. The heat generated melts the floating waste/frit mixture. As glass is melted and poured into canisters 0.61 to $0.91 \mathrm{~m}$ (2 to $3 \mathrm{ft}$ ) in dia to cool, more waste/frit mixture is fed to the melter. Figure 11 shows the LFCM process operated at PNL. Details of equipment design and operation are provided by Buelt and Chapman (1978; 1979).

The LFCM may process simulated waste in either a dry powder/calcine or liquid form. The feeding of a liquid waste stream into a joule-heated melter is expected to become the reference vitrification process for the SRP program. The first two LFCM melting trials, SRL-13 and SRL-14, utilized dry TDS waste premixed with frit. This was done to evaluate the impact of improved feed mixing on the product. The third trial, SRL-LF-1, processed the TDS liquid waste stream directly without prior calcination. Molten glass was regularly sampled from the pouring stream to monitor product composition. Glass samples were also core-drilled from cooled canisters. Feed samples were occasionally taken and analyzed. The results from each melting trial will be presented separately.

\section{SRL-13 (JULY 1979)}

During 5 continuous days of operation, LFCM melting trial SRL-13 filled three canisters with a total of $4250 \mathrm{~kg}$ of TDS-211 waste glass. The objective was to determine the maximum production rate in the LFCM when feeding a wellmixed calcine/frit batch. The maximum melting rate of $70 \mathrm{~kg} / \mathrm{h}$ and average rate of $52 \mathrm{~kg} / \mathrm{h}\left(50 \mathrm{~kg} / \mathrm{h} / \mathrm{m}^{2}\right)$ were limited by the production of $\mathrm{glass}$ foam within the melter. Feeding was stopped during the trial until the foam had dissipated.

The TDS liquid waste used in this trial was spray dried in a $250^{\circ} \mathrm{C}$ to $600^{\circ} \mathrm{C}$ spray drier. The resulting waste powder had a larger weight loss on 


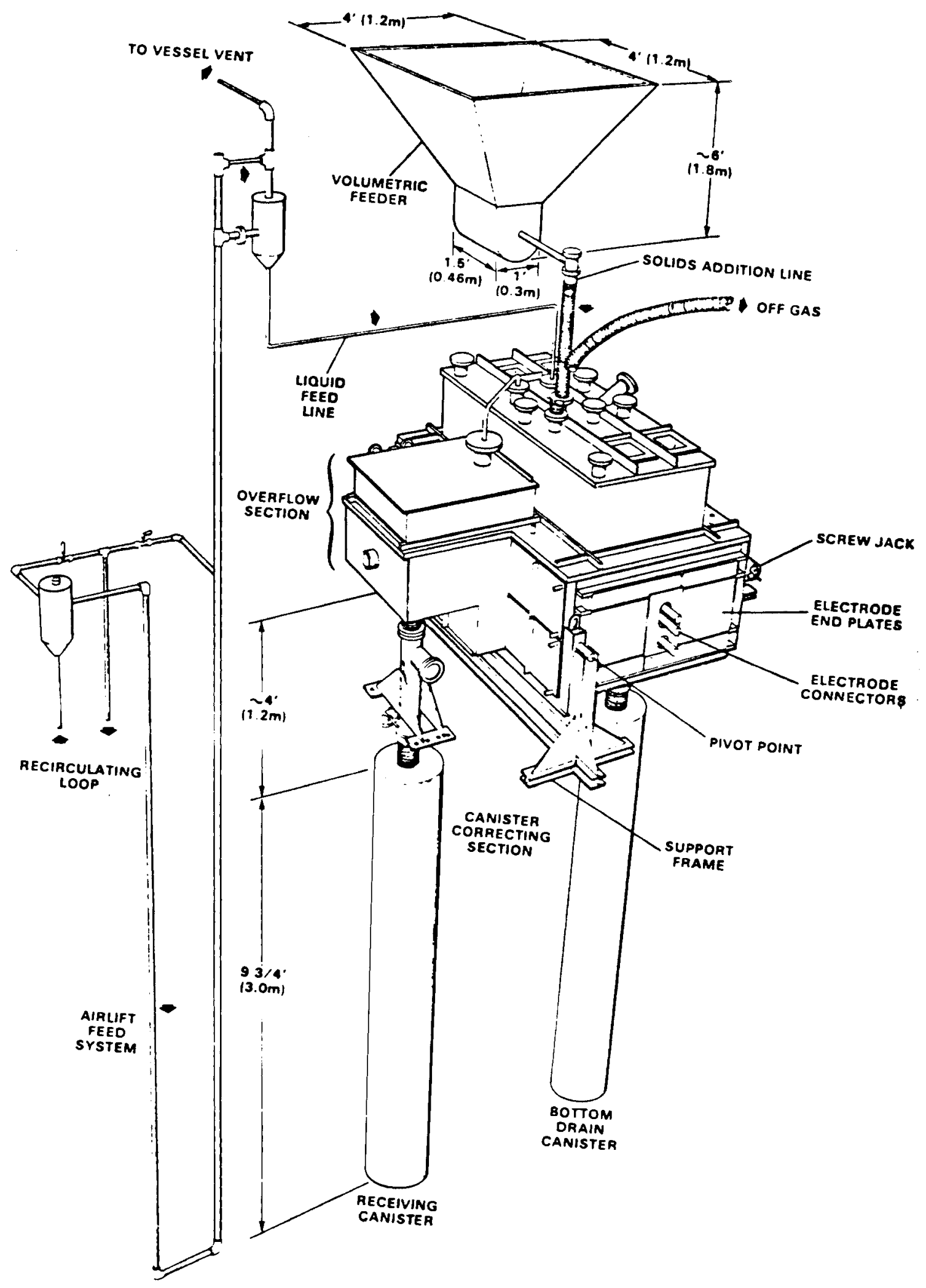

FIGURE 11. Liquid-Fed Ceramic Melter 
heating than calcined waste, $30 \mathrm{wt} \%$ versus $3 \mathrm{wt} \%$. This increased weight loss was not found directly responsible for LFCM foaming. Chemical analys is of liquid and resulting powder indicated that no batching errors occurred in the waste preparation. The dried powder and frit were mixed in barrel mixers to produce a $28 / 72$ calcine/frit oxide ratio before it was fed to the LFCM.

The composition of the glass in the LFCM at the start of SRL-13 was significantly different from TDS-211. Analysis of grab samples collected periodically show the gradual composition change during the trial (Table 25). Figure 12 plots the percent of TDS-211 glass in any sample estimated from $\mathrm{ZnO}$ concentration as a function of glass production. To replace $90 \%$ of the glass in the LFCM with a new composition, it is necessary to process approximately $2500 \mathrm{~kg}$ of glass. When the composition of the starting glass was factored out, an estimate of the TDS-211 glass composition in each sample could be calculated (Table 26). The glass product contained an estimated TDS calcine content of 24 to $27 \mathrm{wt} \%$, only slightly lower than the defined $28 \mathrm{wt} \%$.

Samples were core-drilled from canister 148. Glass in this canister largely represented the feed during this trial and contained $<5 w t \%$ of the original LFCM glass composition. The normalized data for the major constituents are listed in Table 27. The glass in canister 148 shows no significant variation in composition with sample location as seen in Table 28 and had an average annealed glass density of $2.742 \pm 0.007 \mathrm{~g} / \mathrm{cc}$.

Grab samples were always amorphous. Only a trace of an unidentified crystalline phase was detected in some core-drilled canister samples along the bottom and centerline. No significant devitrification occurred on cooling. Neither pH4 nor soxhlet leach results demonstrate significant variations related to sample position within the canister (Tables 29 and 30). Under pH4 conditions, canister 148 samples lost $75.1 \pm 6.0 \mathrm{wt} \%$. Soxhlet conditions produced a weight loss $1.78 \pm 0.12 \mathrm{wt} \%$.

\section{SRL-14 (OCTOBER 1979)}

The previous LFCM melting trial, SRL-13, was hampered by foaming of the calcine/frit feed during melting. Laboratory work indicated that the addition of 1 to 5 wt\% cornstarch as a reducing agent would significantly reduce 
TABLE 25. Normalized Composition SRL-13 Grab Samples

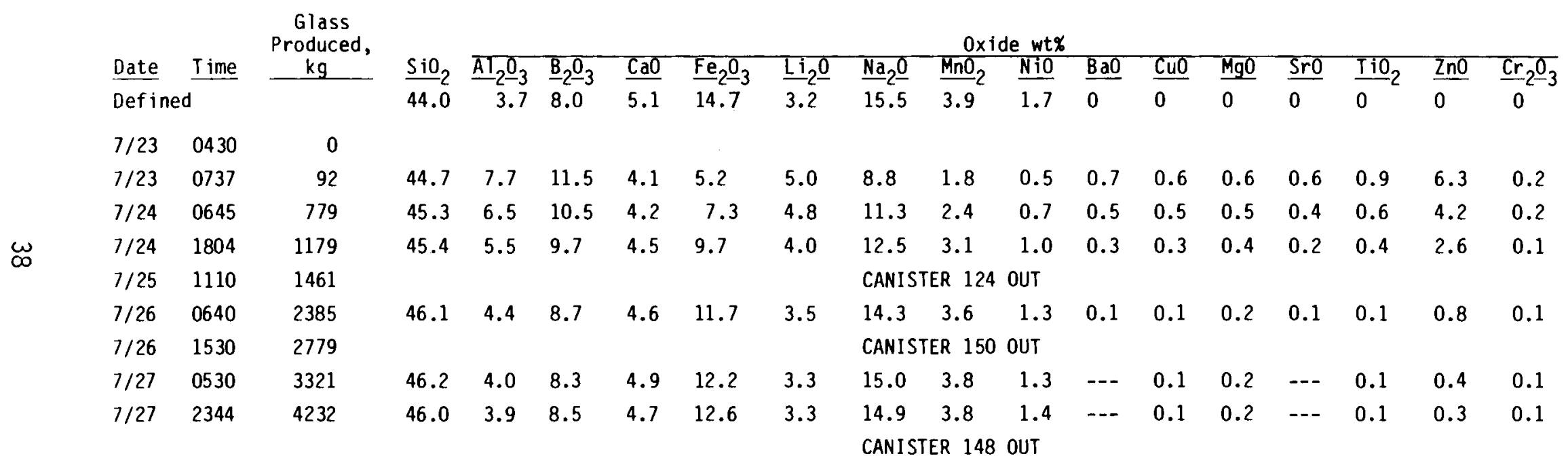




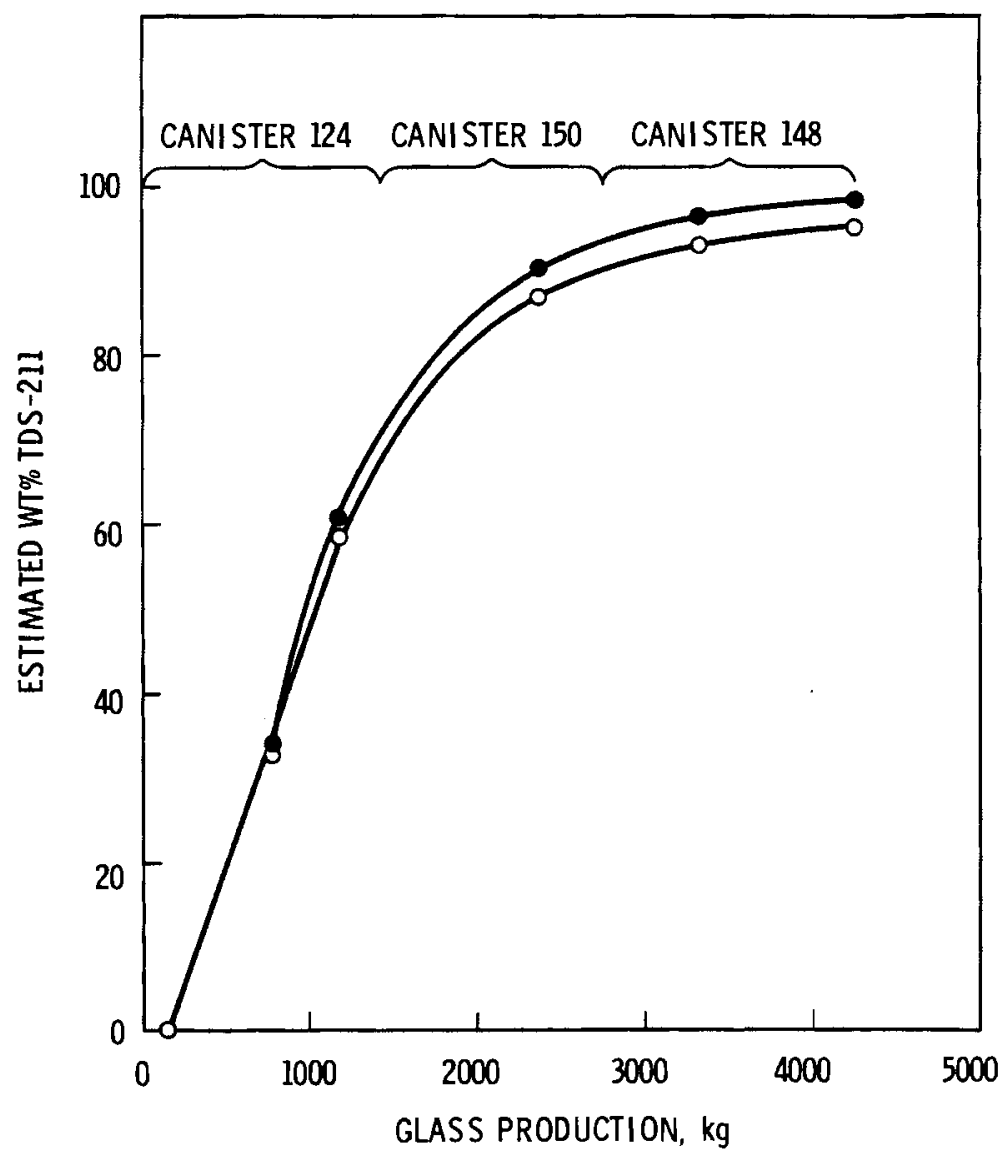

FIGURE 12. TDS-211 Content of SRL-13 Grab Samples

foaming and increase production rates. The objective of melting trial SRL-14 was to determine the optimum cornstarch loading for the SRL-13 batch. During $3 \mathrm{~d}$ of operation, one $0.91-\mathrm{m}$ (36-in.)-dia canister was filled with $2480 \mathrm{~kg}$ of glass at an average sustained rate of $92 \mathrm{~kg} / \mathrm{h}\left(92 \mathrm{~kg} / \mathrm{h} / \mathrm{m}^{2}\right)$. Foaming was drastically reduced by the addition of $1 \mathrm{wt} \%$ cornstarch. Product samples were not analyzed for this run.

SRL-LF-1 (JANUARY 1980)

In January 1980 the LFCM was run for $3 d$ by directly feeding simulated TDS-liquid waste and frit. The objectives of the run were to evaluate the performance of the LFCM process with direct liquid feeding and the properties 


\section{TABLE 26. Est imated TDS-211 Composition (SRL-13)}

\begin{tabular}{|c|c|c|c|c|c|c|c|c|c|c|c|}
\hline Samples & & Per & & & & & $w t$ & & & & \\
\hline (Date) & Time & TDS-2 & $\overline{\mathrm{SiO}_{2}}$ & $\underline{A l}_{2}{ }_{-3}$ & $\overrightarrow{\mathrm{B}_{2}-}-3$ & $\mathrm{CaO}$ & $\mathrm{Fe}_{2} \underline{O}_{3}$ & $\underline{L i}_{2} \underline{0}$ & $\underline{\mathrm{Na}}_{2} \underline{0}$ & $\underline{\mathrm{MnO}}_{2}$ & $\mathrm{~N}$ \\
\hline Defined & & & 44.0 & 3.7 & 8.0 & 5.1 & 14.7 & 3.2 & 15.5 & 3.9 & 1. \\
\hline $7 / 24$ & 0645 & 34.4 & 46.3 & 4.2 & 8.4 & 4.5 & 11.3 & 4.5 & 16.1 & 3.6 & 1. \\
\hline $7 / 24$ & 1804 & 60.6 & 46.0 & 3.9 & 8.5 & 4.7 & 12.9 & 3.2 & 15.2 & 4.1 & 1.3 \\
\hline $7 / 26$ & 0604 & 90.2 & 46.5 & 3.9 & 8.3 & 4.7 & 12.7 & 3.3 & 15.2 & 3.9 & 1.6 \\
\hline $7 / 26$ & 1530 & 96.7 & 46.5 & 3.7 & 8.1 & 4.9 & 12.7 & 3.2 & 15.4 & 4.0 & 1.4 \\
\hline $7 / 27$ & 2344 & 98.4 & 46.2 & 3.7 & 8.4 & 4.7 & 13.0 & 3.3 & 15.2 & 3.9 & 1.5 \\
\hline $\begin{array}{l}\text { AVE. } \\
\text { STD. }\end{array}$ & & & $\begin{array}{r}46.3 \\
0.2\end{array}$ & $\begin{array}{l}3.9 \\
0.2\end{array}$ & $\begin{array}{l}8.3 \\
0.2\end{array}$ & $\begin{array}{l}4.7 \\
0.1\end{array}$ & $\begin{array}{r}12.5 \\
0.7\end{array}$ & $\begin{array}{l}3.5 \\
0.6\end{array}$ & $\begin{array}{r}15.4 \\
0.4\end{array}$ & $\begin{array}{l}3.9 \\
0.2\end{array}$ & \\
\hline
\end{tabular}

TABLE 27. Normalized Composition Of Canister 148 Samples

\begin{tabular}{|c|c|c|c|c|c|c|c|c|c|c|c|c|}
\hline \multicolumn{2}{|c|}{$\begin{array}{l}\text { Core } \\
\text { Height }\end{array}$} & \multicolumn{2}{|c|}{ Core Depth } & \multicolumn{9}{|c|}{ Oxide wt\% } \\
\hline m & $f t$ & $\mathrm{~cm}$ & in. & $\mathrm{SiO}_{2}$ & $\underline{A}_{2}{ }_{2}{ }_{3}$ & $\underline{B}_{2} \underline{O}_{3}$ & $\mathrm{CaO}$ & $\mathrm{Fe}_{2} \mathrm{O}_{3}$ & $\mathrm{Li}_{2} \underline{\underline{O}}$ & $\mathrm{NaO}_{2}$ & $\mathrm{MnO}_{2}$ & $\underline{\mathrm{NiO}}$ \\
\hline Define & & & & 44.0 & 3.7 & 8.0 & 5.1 & 14.7 & 3.2 & 15.5 & 3.9 & 1.7 \\
\hline 0 & 0 & $\begin{array}{l}0-7.6 \\
7.6-15.2 \\
15.2-22.9 \\
22.9-30.5\end{array}$ & $\begin{array}{l}0-3 \\
3-6 \\
6-9 \\
9-12\end{array}$ & $\begin{array}{l}46.4 \\
46.1 \\
46.4 \\
46.5\end{array}$ & $\begin{array}{l}4.1 \\
4.0 \\
4.0 \\
4.0\end{array}$ & $\begin{array}{l}8.5 \\
8.2 \\
8.4 \\
8.3\end{array}$ & $\begin{array}{l}4.9 \\
4.7 \\
5.1 \\
5.1\end{array}$ & $\begin{array}{l}12.2 \\
12.0 \\
12.2 \\
12.2\end{array}$ & $\begin{array}{l}3.3 \\
3.4 \\
3.2 \\
3.2\end{array}$ & $\begin{array}{l}14.6 \\
15.5 \\
14.6 \\
14.5\end{array}$ & $\begin{array}{l}3.8 \\
3.7 \\
3.8 \\
3.8\end{array}$ & $\begin{array}{l}1.3 \\
1.3 \\
1.3 \\
1.3\end{array}$ \\
\hline 0.30 & 1 & $\begin{array}{l}0-7.6 \\
7.6-15.2 \\
15.2-22.9 \\
22.9-30.5\end{array}$ & $\begin{array}{l}0-3 \\
3-6 \\
6-9 \\
9-12\end{array}$ & $\begin{array}{l}46.5 \\
46-2 \\
46.3 \\
46.4\end{array}$ & $\begin{array}{l}4.0 \\
4.0 \\
4.0 \\
4.0\end{array}$ & $\begin{array}{l}8.3 \\
8.3 \\
8.3 \\
8.4\end{array}$ & $\begin{array}{l}5.0 \\
4.9 \\
5.0 \\
5.1\end{array}$ & $\begin{array}{l}12.2 \\
11.9 \\
12.7 \\
12.2\end{array}$ & $\begin{array}{l}3.2 \\
3.4 \\
3.2 \\
3.2\end{array}$ & $\begin{array}{l}14.5 \\
15.4 \\
14.5 \\
14.6\end{array}$ & $\begin{array}{l}3.8 \\
3.7 \\
3.8 \\
3.8\end{array}$ & $\begin{array}{l}1.3 \\
1.2 \\
1.2 \\
1.3\end{array}$ \\
\hline 0.61 & 2 & $\begin{array}{l}0-7.6 \\
7.6-15.2 \\
15.2-22.9 \\
22.9-30.5\end{array}$ & $\begin{array}{l}0-3 \\
3-6 \\
6-9 \\
9-12\end{array}$ & $\begin{array}{l}46.2 \\
45.8 \\
46.1 \\
45.9\end{array}$ & $\begin{array}{l}3.9 \\
3.9 \\
3.9 \\
4.0\end{array}$ & $\begin{array}{l}8.4 \\
8.2 \\
8.3 \\
8.3\end{array}$ & $\begin{array}{l}5.0 \\
4.9 \\
5.0 \\
5.0\end{array}$ & $\begin{array}{l}12.3 \\
11.9 \\
12.2 \\
12.4\end{array}$ & $\begin{array}{l}3.3 \\
3.3 \\
3.2 \\
3.3\end{array}$ & $\begin{array}{l}14.8 \\
14.9 \\
15.1 \\
15.0\end{array}$ & $\begin{array}{l}3.8 \\
3.7 \\
3.8 \\
3.8\end{array}$ & $\begin{array}{l}1.3 \\
1.3 \\
1.3 \\
1.3\end{array}$ \\
\hline 0.91 & 3 & $\begin{array}{l}0-7.6 \\
7.6-15.2 \\
15.2-22.9 \\
22.9-30.5\end{array}$ & $\begin{array}{l}0-3 \\
3-6 \\
6-9 \\
9-12\end{array}$ & $\begin{array}{l}46.3 \\
46.0 \\
45.7 \\
46.1\end{array}$ & $\begin{array}{l}4.0 \\
4.0 \\
4.0 \\
4.0\end{array}$ & $\begin{array}{l}8.4 \\
8.2 \\
8.1 \\
8.2\end{array}$ & $\begin{array}{l}5.0 \\
4.9 \\
4.9 \\
5.1\end{array}$ & $\begin{array}{l}12.4 \\
12.0 \\
12.3 \\
12.7\end{array}$ & $\begin{array}{l}3.1 \\
3.3 \\
3.4 \\
3.1\end{array}$ & $\begin{array}{l}14.7 \\
15.6 \\
15.6 \\
14.6\end{array}$ & $\begin{array}{l}3.8 \\
3.7 \\
3.7 \\
3.9\end{array}$ & $\begin{array}{l}1.3 \\
1.3 \\
1.3 \\
1.3\end{array}$ \\
\hline 1.21 & 4 & $\begin{array}{l}0-7.6 \\
7.6-15.2 \\
15.2-22.9 \\
22.9-30.5\end{array}$ & $\begin{array}{l}0-3 \\
3-6 \\
6-9 \\
9-12\end{array}$ & $\begin{array}{l}46.2 \\
46.1 \\
45.7 \\
45.7\end{array}$ & $\begin{array}{l}3.9 \\
3.9 \\
3.9 \\
3.9\end{array}$ & $\begin{array}{l}8.2 \\
8.3 \\
8.3 \\
8.4\end{array}$ & $\begin{array}{l}5.1 \\
5.0 \\
4.9 \\
5.0\end{array}$ & $\begin{array}{l}12.5 \\
12.4 \\
12.1 \\
12.2\end{array}$ & $\begin{array}{l}3.2 \\
3.3 \\
3.3 \\
3.2\end{array}$ & $\begin{array}{l}14.7 \\
14.8 \\
15.7 \\
15.4\end{array}$ & $\begin{array}{l}3.8 \\
3.8 \\
3.8 \\
3.8\end{array}$ & $\begin{array}{l}1.3 \\
1.3 \\
1.3 \\
1.2\end{array}$ \\
\hline 1.52 & 5 & $\begin{array}{l}0-7.6 \\
7.6-15.2 \\
15.2-22.9 \\
22.9-30.5\end{array}$ & $\begin{array}{l}0-3 \\
3-6 \\
6-9 \\
9-12\end{array}$ & $\begin{array}{l}45.3 \\
46.0 \\
45.5 \\
46.4\end{array}$ & $\begin{array}{l}4.0 \\
3.8 \\
4.0 \\
3.9\end{array}$ & $\begin{array}{l}8.2 \\
8.4 \\
8.3 \\
8.3\end{array}$ & $\begin{array}{l}4.8 \\
4.9 \\
4.8 \\
5.0\end{array}$ & $\begin{array}{l}12.2 \\
12.0 \\
11.9 \\
12.1\end{array}$ & $\begin{array}{l}3.2 \\
3.3 \\
3.3 \\
3.3\end{array}$ & $\begin{array}{l}16.2 \\
15.6 \\
16.3 \\
15.1\end{array}$ & $\begin{array}{l}3.8 \\
3.7 \\
3.7 \\
3.8\end{array}$ & $\begin{array}{l}1.3 \\
1.2 \\
1.2 \\
1.1\end{array}$ \\
\hline 1.83 & 6 & $\begin{array}{l}0-7.6 \\
7.6-15.2\end{array}$ & $\begin{array}{l}0-3 \\
3-6\end{array}$ & $\begin{array}{l}46.2 \\
46.2\end{array}$ & $\begin{array}{l}3.8 \\
3.9\end{array}$ & $\begin{array}{l}8.3 \\
8.3\end{array}$ & $\begin{array}{l}5.1 \\
4.9\end{array}$ & $\begin{array}{l}12.5 \\
11.7\end{array}$ & $\begin{array}{l}3.2 \\
3.5\end{array}$ & $\begin{array}{l}15.0 \\
15.7\end{array}$ & $\begin{array}{l}3.7 \\
3.7\end{array}$ & $\begin{array}{l}1.2 \\
1.1\end{array}$ \\
\hline
\end{tabular}


TABLE 28. Average Composition of Canister 148 Samples

Core Height

\begin{tabular}{|c|c|c|c|c|c|c|c|c|c|c|}
\hline \multicolumn{2}{|c|}{ Core Height } & \multicolumn{9}{|c|}{ Oxide wt\% } \\
\hline $\mathrm{m}$ & $\mathrm{ft}$ & $\overline{\mathrm{SiO}_{2}}$ & $\mathrm{Al}_{2} \underline{O}_{3}$ & $\underline{B}_{2}-\underline{O}_{3}$ & $\mathrm{CaO}$ & $\mathrm{Fe}_{2}{ }_{-}{ }_{3}$ & $\mathrm{Li}_{2} \underline{ } \underline{ }$ & $\underline{\mathrm{Na}}_{2} 2 \underline{0}$ & $\mathrm{MnO}_{2}$ & $\mathrm{NiO}$ \\
\hline 0 & 0 & 46.4 & 4.0 & 8.4 & 5.0 & 12.2 & 3.3 & 14.8 & 3.8 & 1.3 \\
\hline 0.30 & 1 & 46.4 & 4.0 & 8.3 & 5.0 & 12.2 & 3.2 & 14.8 & 3.8 & 1.2 \\
\hline 0.61 & 2 & 46.0 & 3.9 & 8.3 & 5.0 & 12.2 & 3.3 & 15.0 & 3.8 & 1.3 \\
\hline 0.91 & 3 & 46.0 & 4.0 & 8.2 & 5.0 & 12.4 & 3.2 & 15.1 & 3.8 & 1.3 \\
\hline 1.21 & 4 & 45.9 & 3.9 & 8.3 & 5.0 & 12.3 & 3.2 & 15.2 & 3.8 & 1.3 \\
\hline 1.52 & 5 & 45.8 & 3.9 & 8.3 & 4.9 & 12.0 & 3.3 & 15.8 & 3.8 & 1.2 \\
\hline 1.83 & 6 & 46.2 & 3.8 & 8.3 & 5.0 & 12.1 & 3.4 & 15.3 & 3.7 & 1.2 \\
\hline
\end{tabular}

Core Depth

\begin{tabular}{|c|c|c|c|c|c|c|c|c|c|}
\hline $0-7.6$ & $0-3$ & 46.2 & 4.0 & 8.3 & 5.0 & 12.3 & 3.2 & 14.9 & 3.8 \\
\hline $7.6-15.2$ & $3-6$ & 46.0 & 3.9 & 8.3 & 4.9 & 12.0 & 3.4 & 15.3 & 3.7 \\
\hline $15.2-22.9$ & $6-9$ & 46.0 & 4.0 & 8.3 & 5.0 & 12.2 & 3.4 & 15.4 & 3.8 \\
\hline $22.9-30.5$ & $9-12$ & 46.2 & 4.0 & 8.3 & 5.0 & 12.3 & 3.2 & 14.9 & 3.8 \\
\hline $\begin{array}{l}\text { AVE. } \\
\text {;TD. DEV. }\end{array}$ & & $\begin{array}{r}46.1 \\
0.3\end{array}$ & $\begin{array}{l}4.0 \\
0.1\end{array}$ & $\begin{array}{l}8.3 \\
0.1\end{array}$ & $\begin{array}{l}5.0 \\
0.1\end{array}$ & $\begin{array}{r}12.2 \\
0.2\end{array}$ & $\begin{array}{l}3.3 \\
0.1\end{array}$ & $\begin{array}{l}15.1 \\
<0.5\end{array}$ & $\begin{array}{r}3.8 \\
<0.1\end{array}$ \\
\hline
\end{tabular}

TABLE 29. Weight Loss of Canister 148 Sample Under pH4 Conditions

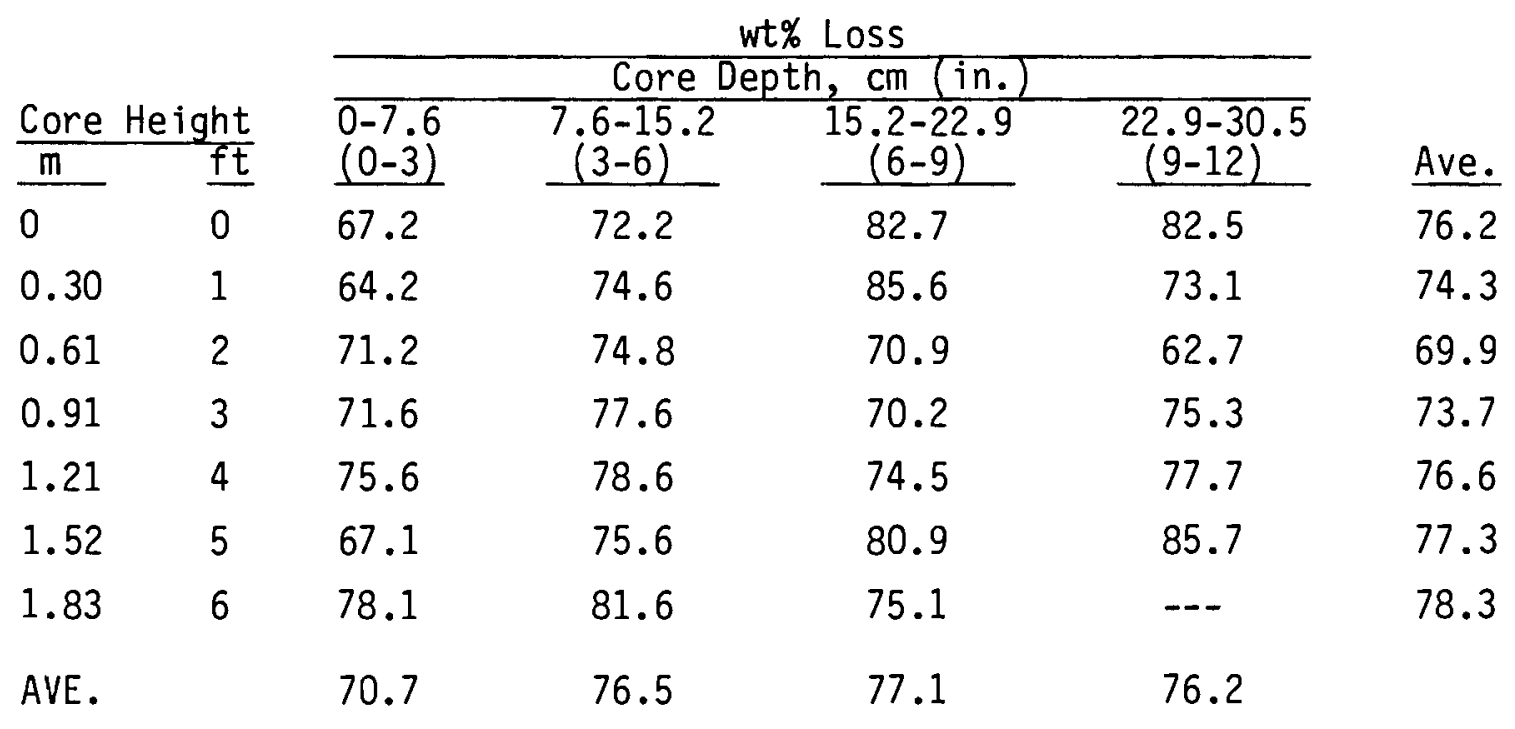

Mean pH4 Weight Loss $75.1 \pm 6.0 \mathrm{wt} \%$. 
TABLE 30. Weight Loss of Canister 148 Sample Under Soxhlet Conditions

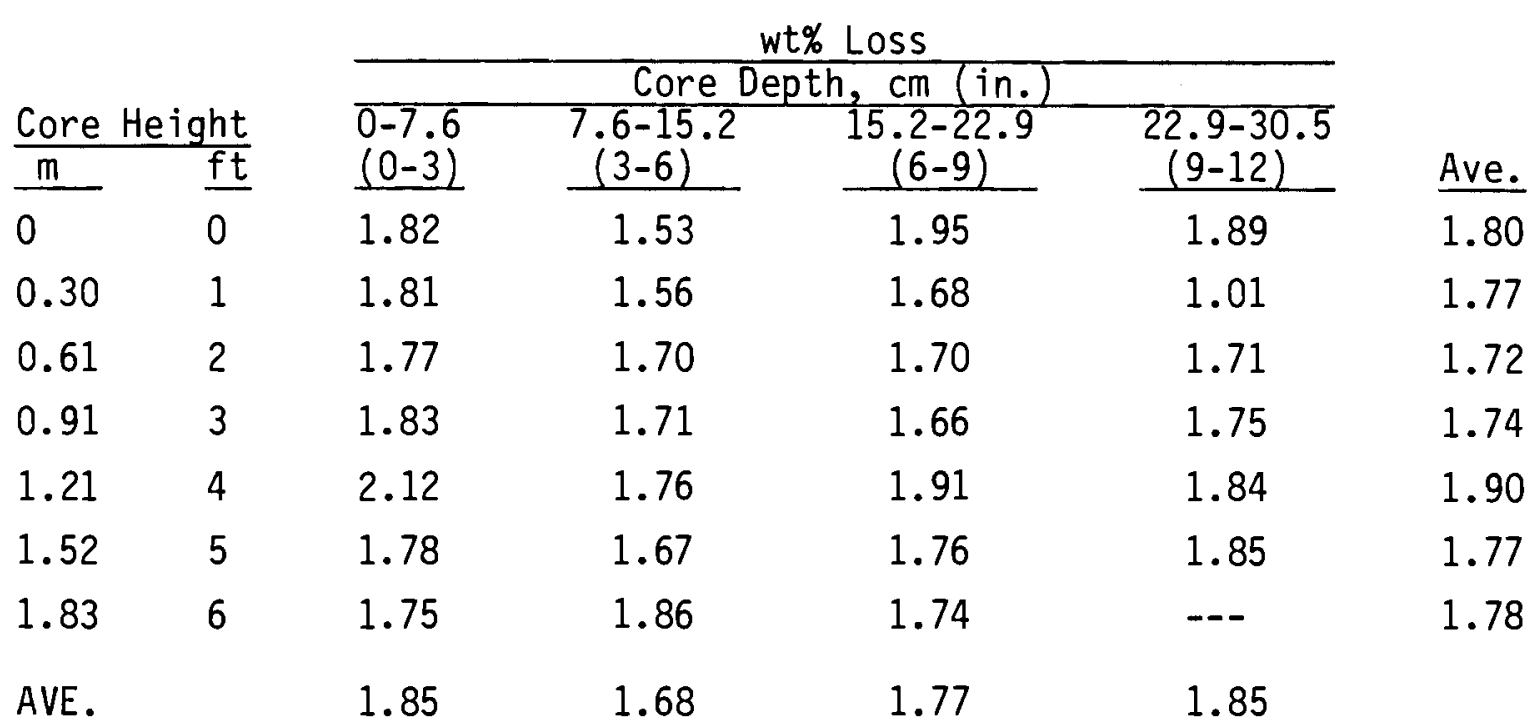

Mean Soxhlet Weight Loss $1.78 \pm 0.12$ wt\%

of the resulting product. Approximately $2900 \mathrm{~L}$ of waste slurry were converted to $\cong 1050 \mathrm{~kg}$ of waste $\mathrm{glass}$ at a maximum sustained glass production rate of $40 \mathrm{~kg} / \mathrm{h}\left(38 \mathrm{~kg} / \mathrm{h} / \mathrm{m}^{2}\right)$.

The waste slurry was a mixture of TDS simulated waste and -200 mesh 211 frit in a 28:72 oxide ratio. Bentonite and cornstarch were added to keep solids in suspension and reduce foaming, respectively. The feed was also spiked with volatile components $\mathrm{Ru}, \mathrm{Sb}, \mathrm{Te}$ and $\mathrm{Cs}$ to examine their behavior under processing conditions. The composition of the liquid feed is listed in Table 31. Problems with foaming initially lowered production rates; however, plugging and erosion of the feed system was a more persistent problem throughout the run causing several equipment stoppages. Glass melting rates as a result varied considerably.

Samples of liquid feed were regularly collected and analyzed. The liquid feed oxide composition and oxide loading are listed in Table 32. The average feed composition approximated the target values moderately we11. No cause was identified, however, for discrepancies in $\mathrm{B}_{2} \mathrm{O}_{3}$ and $\mathrm{Li}_{2} \mathrm{O}$ content. Since both are frit constituents, they are usually both above or below target. The large variation in $\mathrm{MnO}_{2}$ content again reflects normal sampling error for this 
TABLE 31. SRL-LF-1 Liquid Feed Makeup

Compound

$\frac{\text { Waste }}{\mathrm{Fe}(\mathrm{OH})_{3}}$
$\mathrm{Al}(\mathrm{HO})_{3}$
$\mathrm{MnO}_{2}$
$\mathrm{Ni}_{(\mathrm{OH})_{2}}$
$\mathrm{CaCO}_{3}$
$\mathrm{NaNO}_{3}$
$\mathrm{Na}_{2} \mathrm{SO}_{4}$
$\mathrm{ZeOlite}^{(\mathrm{a})}$

$\mathrm{RuNO}\left(\mathrm{NO}_{3}\right)_{3}$

$\mathrm{Sb}_{2} \mathrm{O}_{3}$

$\mathrm{TeO}_{2}$

$\mathrm{Cs}_{2} \mathrm{CO}_{3}$

$\mathrm{Sr}\left(\mathrm{NO}_{3}\right)_{2}$
Concentration

$(g / L)$

70.0

17.5

14.0

7.5

6.4

2.9

1.4

10.4

0.204

0.079

0.024

0.237

0.166

Additives, wt\%

$\begin{array}{lrl}\mathrm{SiO}_{2} & 58.3 & \\ \mathrm{Na}_{2} \mathrm{O} & 20.6 & \text { Premelted } \\ \mathrm{B}_{2} \mathrm{O}_{3} & 11.1 & \text { Frit-211 } \\ \mathrm{CaO} & 5.6 & (-200 \text { mesh }) \\ \mathrm{Li}_{2} \mathrm{O} & 4.4 & 258 \\ \text { Cornstarch }\left(\mathrm{C}_{6} \mathrm{H}_{10} \mathrm{O}_{5}\right) \mathrm{n} & \\ \begin{array}{l}\text { Bentonite } \\ \text { TOTAL }\end{array} \\ \begin{array}{l}\text { Total } \\ \text { R }\end{array}\end{array}$

(a) Linde Ion SiV IE-95, 2

(b) Alumina silicate clay assumed to be $\mathrm{Al}_{2} \mathrm{O}_{3} \cdot 4 \mathrm{SiO}_{2} \cdot \mathrm{H}_{2} \mathrm{O}$ 
TABLE 32. Composition of SRL-LF-1 Liquid Feed Samples

\begin{tabular}{|c|c|c|c|c|c|c|c|c|c|c|c|c|c|c|c|c|c|}
\hline & \multicolumn{16}{|c|}{ 0xide wt\% } & \multirow{2}{*}{$\begin{array}{l}\text { Total 0xides } \\
\text { g/L Feed } \\
\end{array}$} \\
\hline & Time & $\underline{\mathrm{SiO}}_{2}$ & $\underline{A l}_{2}-{ }_{-}$ & $\underline{B}_{2} \underline{0}_{3}$ & $\underline{\mathrm{CaO}}$ & $\underline{\mathrm{Fe}}_{2} \underline{\mathrm{O}}_{3}$ & $\underline{L i}_{2} \underline{0}$ & $\underline{\mathrm{Na}}_{2} \underline{0}$ & $\mathrm{MnO}_{2}$ & $\underline{\mathrm{NiO}}$ & $\underline{\mathrm{RuO}}_{2}$ & $\underline{S b}_{2} \underline{O}_{3}$ & $\mathrm{TeO}_{2-}$ & $\underline{C s}_{2} \underline{0}$ & Sro & $c 1^{-(a)}$ & \\
\hline & Defined & 44.5 & 4.2 & 7.8 & 5.0 & 14.4 & 3.1 & 14.9 & $3.8^{2}$ & 1.6 & 0.023 & 0.022 & 0.006 & 0.056 & 0.022 & 0.29 & 400.0 \\
\hline $1 / 14$ & $\begin{array}{l}2140 \\
2330\end{array}$ & $\begin{array}{l}43.6 \\
42.9\end{array}$ & $\begin{array}{l}4.4 \\
3.8\end{array}$ & $\begin{array}{l}8.2 \\
9.9\end{array}$ & $\begin{array}{l}5.1 \\
4.7\end{array}$ & $\begin{array}{l}13.0 \\
12.0\end{array}$ & $\begin{array}{l}2.5 \\
2.7\end{array}$ & $\begin{array}{l}14.7 \\
16.8\end{array}$ & $\begin{array}{l}4.4 \\
3.3\end{array}$ & $\begin{array}{l}1.5 \\
1.4\end{array}$ & $\begin{array}{l}0.301 \\
0.290\end{array}$ & $\begin{array}{l}0.084 \\
0.078\end{array}$ & $\begin{array}{l}0.073 \\
0.092\end{array}$ & $\begin{array}{l}0.054 \\
0.050\end{array}$ & $\begin{array}{l}0.038 \\
0.040\end{array}$ & $\begin{array}{l}1.39 \\
1.32\end{array}$ & $\begin{array}{l}336.8 \\
328.3\end{array}$ \\
\hline $1 / 15$ & $\begin{array}{l}0145 \\
0330 \\
0630 \\
0908 \\
1105 \\
1250\end{array}$ & $\begin{array}{l}43.4 \\
43.3 \\
44.1 \\
44.0 \\
43.8 \\
44.3\end{array}$ & $\begin{array}{l}4.0 \\
3.8 \\
4.3 \\
4.2 \\
4.2 \\
4.4\end{array}$ & $\begin{array}{l}9.5 \\
10.2 \\
8.4 \\
8.4 \\
8.5 \\
8.4\end{array}$ & $\begin{array}{l}4.8 \\
4.8 \\
5.3 \\
5.3 \\
5.2 \\
5.2\end{array}$ & $\begin{array}{l}11.7 \\
12.3 \\
13.6 \\
13.6 \\
13.5 \\
13.4\end{array}$ & $\begin{array}{l}2.6 \\
2.6 \\
2.5 \\
2.6 \\
2.5 \\
2.5\end{array}$ & $\begin{array}{l}15.8 \\
16.3 \\
14.6 \\
14.8 \\
14.5 \\
14.3\end{array}$ & $\begin{array}{l}3.5 \\
2.7 \\
3.2 \\
3.2 \\
3.8 \\
3.5\end{array}$ & $\begin{array}{l}1.4 \\
1.4 \\
1.6 \\
1.6 \\
1.6 \\
1.6\end{array}$ & $\begin{array}{l}0.328 \\
0.309 \\
0.301 \\
0.323 \\
0.299 \\
0.326\end{array}$ & $\begin{array}{l}0.080 \\
0.080 \\
0.085 \\
0.083 \\
0.083 \\
0.087\end{array}$ & $\begin{array}{l}0.082 \\
0.090 \\
0.073 \\
0.081 \\
0.078 \\
0.071\end{array}$ & $\begin{array}{l}0.055 \\
0.051 \\
0.054 \\
0.054 \\
0.055 \\
0.060\end{array}$ & $\begin{array}{l}0.039 \\
0.042 \\
0.038 \\
0.037 \\
0.039 \\
0.040\end{array}$ & $\begin{array}{l}1.36 \\
1.33 \\
1.30 \\
1.30 \\
1.31 \\
1.35\end{array}$ & $\begin{array}{l}335.6 \\
344.8 \\
341.8 \\
341.4 \\
350.6 \\
351.0\end{array}$ \\
\hline $1 / 16$ & $\begin{array}{l}0030 \\
0230 \\
0430 \\
1710 \\
2222\end{array}$ & $\begin{array}{l}44.1 \\
43.9 \\
44.0 \\
44.2 \\
44.2\end{array}$ & $\begin{array}{l}4.2 \\
4.3 \\
4.4 \\
4.2 \\
4.1\end{array}$ & $\begin{array}{l}8.5 \\
8.3 \\
8.2 \\
8.4 \\
8.4\end{array}$ & $\begin{array}{l}5.3 \\
5.2 \\
5.3 \\
5.3 \\
4.6\end{array}$ & $\begin{array}{l}13.7 \\
13.4 \\
13.6 \\
13.7 \\
13.8\end{array}$ & $\begin{array}{l}2.4 \\
2.6 \\
2.6 \\
2.7 \\
2.6\end{array}$ & $\begin{array}{l}13.9 \\
14.0 \\
14.4 \\
13.9 \\
14.7\end{array}$ & $\begin{array}{l}3.9 \\
4.3 \\
3.6 \\
2.9 \\
2.7\end{array}$ & $\begin{array}{l}1.5 \\
1.6 \\
1.6 \\
1.5 \\
1.6\end{array}$ & $\begin{array}{l}0.327 \\
0.299 \\
0.320 \\
0.330 \\
0.322\end{array}$ & $\begin{array}{l}0.088 \\
0.083 \\
0.086 \\
0.085 \\
0.090\end{array}$ & $\begin{array}{l}0.081 \\
0.067 \\
0.066 \\
0.077 \\
0.078\end{array}$ & $\begin{array}{l}0.056 \\
0.055 \\
0.056 \\
0.054 \\
0.050\end{array}$ & $\begin{array}{l}0.038 \\
0.038 \\
0.037 \\
0.036 \\
0.038\end{array}$ & $\begin{array}{l}1.38 \\
1.34 \\
1.38 \\
1.36 \\
1.37\end{array}$ & $\begin{array}{l}304.7 \\
447.4 \\
340.9 \\
334.6 \\
332.9\end{array}$ \\
\hline $1 / 7$ & $\begin{array}{l}0020 \\
0125 \\
0230 \\
0335 \\
0425 \\
0525 \\
0630 \\
0755 \\
1015 \\
1245 \\
1400 \\
1500\end{array}$ & $\begin{array}{l}44.1 \\
44.6 \\
44.8 \\
45.0 \\
44.6 \\
44.5 \\
44.2 \\
43.9 \\
43.8 \\
43.6 \\
44.1 \\
44.6\end{array}$ & $\begin{array}{l}4.3 \\
4.2 \\
4.2 \\
4.2 \\
4.2 \\
4.2 \\
4.1 \\
4.2 \\
4.1 \\
4.2 \\
4.0 \\
4.0\end{array}$ & $\begin{array}{l}8.3 \\
8.6 \\
8.4 \\
8.6 \\
8.5 \\
8.6 \\
8.4 \\
8.2 \\
8.2 \\
8.2 \\
8.4 \\
8.4\end{array}$ & $\begin{array}{l}5.3 \\
5.3 \\
5.4 \\
5.5 \\
5.4 \\
5.2 \\
4.9 \\
5.0 \\
4.8 \\
5.3 \\
5.0 \\
5.2\end{array}$ & $\begin{array}{l}13.6 \\
13.8 \\
14.0 \\
14.3 \\
14.0 \\
13.7 \\
13.5 \\
13.5 \\
13.6 \\
14.0 \\
13.9 \\
14.4\end{array}$ & $\begin{array}{l}2.5 \\
2.6 \\
2.5 \\
2.6 \\
2.6 \\
2.9 \\
2.9 \\
2.8 \\
2.8 \\
2.6 \\
2.7 \\
2.6\end{array}$ & $\begin{array}{l}14.5 \\
14.7 \\
14.1 \\
14.4 \\
15.1 \\
16.0 \\
16.1 \\
15.4 \\
15.6 \\
15.0 \\
15.0 \\
15.2\end{array}$ & $\begin{array}{l}3.3 \\
2.2 \\
2.7 \\
1.2 \\
1.5 \\
1.2 \\
1.9 \\
2.9 \\
3.0 \\
3.1 \\
2.9 \\
1.5\end{array}$ & $\begin{array}{l}1.6 \\
1.5 \\
1.6 \\
1.6 \\
1.6 \\
1.5 \\
1.5 \\
1.5 \\
1.5 \\
1.5 \\
1.6 \\
1.5\end{array}$ & $\begin{array}{l}0.302 \\
0.338 \\
0.338 \\
0.340 \\
0.328 \\
0.326 \\
0.275 \\
0.248 \\
0.311 \\
0.330 \\
0.284 \\
0.290\end{array}$ & $\begin{array}{l}0.082 \\
0.087 \\
0.090 \\
0.088 \\
0.083 \\
0.086 \\
0.069 \\
0.070 \\
0.080 \\
0.083 \\
0.075 \\
0.079\end{array}$ & $\begin{array}{l}0.071 \\
0.073 \\
0.073 \\
0.074 \\
0.071 \\
0.076 \\
0.060 \\
0.058 \\
0.070 \\
0.080 \\
0.066 \\
0.072\end{array}$ & $\begin{array}{l}0.054 \\
0.051 \\
0.052 \\
0.046 \\
0.046 \\
0.047 \\
0.042 \\
0.048 \\
0.048 \\
0.050 \\
0.049 \\
0.048\end{array}$ & $\begin{array}{l}0.038 \\
0.041 \\
0.040 \\
0.040 \\
0.037 \\
0.038 \\
0.034 \\
0.035 \\
0.036 \\
0.035 \\
0.035 \\
0.036\end{array}$ & $\begin{array}{l}1.38 \\
1.33 \\
1.34 \\
1.36 \\
1.34 \\
1.29 \\
1.21 \\
1.26 \\
1.29 \\
1.30 \\
1.36 \\
1.36\end{array}$ & $\begin{array}{l}320.0 \\
321.5 \\
316.2 \\
317.0 \\
331.7 \\
327.5 \\
310.1 \\
324.2 \\
322.0 \\
328.1 \\
315.8 \\
144.0\end{array}$ \\
\hline $\begin{array}{l}\text { AVE. } \\
\text { STD. }\end{array}$ & DEV. & $\begin{array}{r}44.1 \\
0.5\end{array}$ & $\begin{array}{l}4.2 \\
0.2\end{array}$ & $\begin{array}{l}8.6 \\
0.5\end{array}$ & $\begin{array}{l}5.1 \\
0.2\end{array}$ & $\begin{array}{r}13.5 \\
0.6\end{array}$ & $\begin{array}{l}2.6 \\
0.1\end{array}$ & $\begin{array}{r}15.0 \\
0.8\end{array}$ & $\begin{array}{l}2.9 \\
0.9\end{array}$ & $\begin{array}{l}1.5 \\
0.1\end{array}$ & $\begin{array}{l}0.311 \\
0.022\end{array}$ & $\begin{array}{l}0.083 \\
0.055\end{array}$ & $\begin{array}{l}0.074 \\
0.008\end{array}$ & $\begin{array}{l}0.051 \\
0.004\end{array}$ & $\begin{array}{l}0.038 \\
0.002\end{array}$ & $\begin{array}{l}1.34 \\
0.04\end{array}$ & $\begin{array}{r}326.8 \\
46.4\end{array}$ \\
\hline
\end{tabular}

(a) $\mathrm{Cl}^{-}$not separately added to waste. 
constituent. The trace constituents were present at greater levels than anticipated. The $\mathrm{Cl}^{-}$was introduced as a contaminant in the ferric hydroxide at approximately 4.5 times its anticipated level in the actual waste stream.

Grab samples of the glass produced were collected and analyzed during the trial. The compositions of the samples are shown in Table 33. The first sample on $11 / 15 / 80$ at 0917 represents the bulk glass in the LFCM at the start of the run. As noted in Figure 12, the production of $1000 \mathrm{~kg}$ of glass results in a product containing approximately $50 \%$ of the original composition in the me1ter and $50 \%$ of the new composition. This accounts for some of the discrepancies noted between targets and analyzed compositions. The trace constituent analyses are not consistent with intended targets due to larger feed concentrations. The analysis of melter samples before the melting trial showed no significant trace oxide concentrations. Approximately $0.45 \% \mathrm{Cl}^{-}$was analyzed in the glass at the end of the run. This represents about one third of the $\mathrm{Cl}^{-}$ present in the liquid waste feed.

Canister 10 filled near the end of the run when compositional variation was diminished was core-drilled for sample analysis. Table 34 presents the composition of the major constituents in Canister 10 samples. The analysis of canister and grab samples were both close to the defined composition with small standard deviations. The chemical durability of canister samples did not vary with sample location (Tables 35 and 36). The average weight loss of core samples under pH4 and soxhlet conditions were $73.8 \pm 2.3 \mathrm{wt} \%$ and $2.05 \pm 0.21 \mathrm{wt} . \%$, respectively. 
TABLE 33. Normalized Composition of SRL-LF-1 Grab Samples

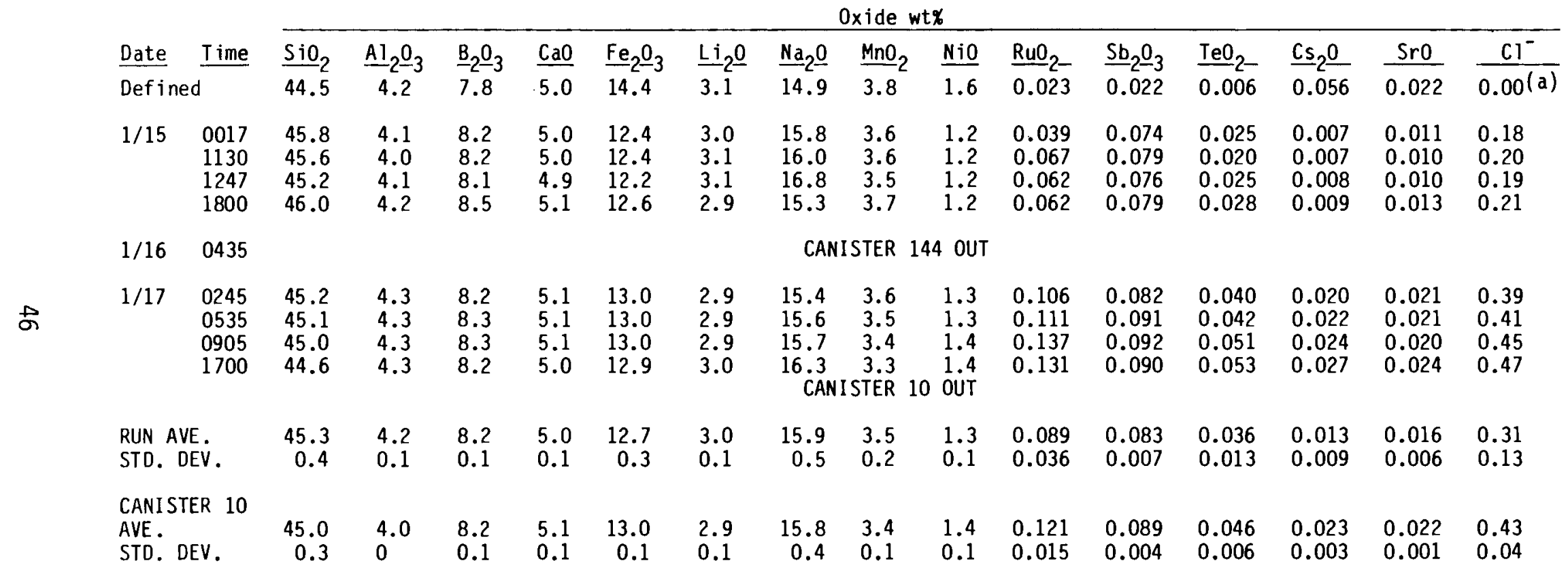

(a) No $\mathrm{Cl}^{-}$expected to enter glass. 
TABLE 34. Normalized Composition of Canister 10 Samples

\begin{tabular}{|c|c|c|c|c|c|c|c|c|c|c|c|c|c|c|}
\hline \multicolumn{2}{|c|}{$\begin{array}{c}\text { Core } \\
\text { Height }\end{array}$} & \multicolumn{2}{|c|}{ Core Depth } & \multicolumn{11}{|c|}{ Oxide wt\% } \\
\hline $\mathrm{m}$ & $\mathrm{ft}$ & $\mathrm{cm}$ & in. & $\underline{\mathrm{SiO}}_{2}$ & $\underline{A l}_{2} \underline{O}_{3}$ & $\underline{B}_{2} \underline{O}_{3}$ & $\underline{\mathrm{CaO}}$ & $\mathrm{Fe}_{2} \underline{\mathrm{O}}_{3}$ & $\underline{\operatorname{Li}} 2 \underline{\underline{O}}$ & $\underline{\mathrm{Na}} 2 \underline{0}$ & $\underline{\mathrm{MnO}}_{2}$ & $\underline{\mathrm{NiO}}$ & Sro & $\mathrm{Cl}^{-}$ \\
\hline$\overline{\text { Defin }}$ & & & & 44.5 & 4.2 & 7.8 & $\overline{5.0}$ & 14.4 & 3.1 & 15.0 & $3.8^{2}$ & $\overline{1.6}$ & 0.02 & 0.29 (a) \\
\hline 0.30 & 1 & $\begin{array}{l}0-7.6 \\
7.6-15.2 \\
15.2-22.9 \\
22.9-30.5\end{array}$ & $\begin{array}{l}0-3 \\
3-6 \\
6-9 \\
9-12\end{array}$ & $\begin{array}{l}45.4 \\
46.3 \\
45.4 \\
45.8\end{array}$ & $\begin{array}{l}4.2 \\
4.4 \\
4.3 \\
4.3\end{array}$ & $\begin{array}{l}7.9 \\
8.0 \\
7.8 \\
7.9\end{array}$ & $\begin{array}{l}5.1 \\
5.1 \\
5.0 \\
5.0\end{array}$ & $\begin{array}{l}13.1 \\
13.3 \\
12.9 \\
13.1\end{array}$ & $\begin{array}{l}2.9 \\
3.1 \\
3.2 \\
3.1\end{array}$ & $\begin{array}{l}15.7 \\
14.0 \\
16.1 \\
15.1\end{array}$ & $\begin{array}{l}3.7 \\
3.7 \\
3.6 \\
3.6\end{array}$ & $\begin{array}{l}1.4 \\
1.4 \\
1.3 \\
1.3\end{array}$ & $\begin{array}{l}0.01 \\
0.01 \\
0.01 \\
0.01\end{array}$ & $\begin{array}{l}0.34 \\
0.35 \\
0.30 \\
0.36\end{array}$ \\
\hline 0.61 & 2 & $\begin{array}{l}0-7.6 \\
7.6-15.2 \\
15.2-22.9 \\
22.9-30.5\end{array}$ & $\begin{array}{l}0-3 \\
3-6 \\
6-9 \\
9-12\end{array}$ & $\begin{array}{l}45.7 \\
45.6 \\
45.7 \\
45.5\end{array}$ & $\begin{array}{l}4.3 \\
4.3 \\
4.3 \\
4.4\end{array}$ & $\begin{array}{l}7.9 \\
7.9 \\
7.9 \\
7.9\end{array}$ & $\begin{array}{l}5.0 \\
5.0 \\
5.0 \\
5.0\end{array}$ & $\begin{array}{l}13.0 \\
13.1 \\
13.2 \\
13.1\end{array}$ & $\begin{array}{l}3.3 \\
3.2 \\
3.1 \\
3.1\end{array}$ & $\begin{array}{l}15.2 \\
15.1 \\
15.1 \\
15.2\end{array}$ & $\begin{array}{l}3.5 \\
3.5 \\
3.5 \\
3.4\end{array}$ & $\begin{array}{l}1.4 \\
1.4 \\
1.4 \\
1.4\end{array}$ & $\begin{array}{l}0.02 \\
0.02 \\
0.02 \\
0.02\end{array}$ & $\begin{array}{l}0.39 \\
0.41 \\
0.36 \\
0.38\end{array}$ \\
\hline 0.91 & 3 & $\begin{array}{l}0-7.6 \\
7.6-15.2 \\
15.2-22.9 \\
22.9-30.5\end{array}$ & $\begin{array}{l}0-3 \\
3-6 \\
6-9 \\
9-12\end{array}$ & $\begin{array}{l}45.6 \\
45.6 \\
45.3 \\
45.5\end{array}$ & $\begin{array}{l}4.3 \\
4.3 \\
4.3 \\
4.4\end{array}$ & $\begin{array}{l}7.9 \\
8.0 \\
7.9 \\
8.0\end{array}$ & $\begin{array}{l}5.1 \\
5.1 \\
5.0 \\
5.1\end{array}$ & $\begin{array}{l}13.5 \\
13.4 \\
13.3 \\
13.3\end{array}$ & $\begin{array}{l}3.0 \\
3.1 \\
3.1 \\
3.0\end{array}$ & $\begin{array}{l}14.7 \\
14.8 \\
15.4 \\
15.0\end{array}$ & $\begin{array}{l}3.4 \\
3.4 \\
3.3 \\
3.4\end{array}$ & $\begin{array}{l}1.5 \\
1.4 \\
1.4 \\
1.4\end{array}$ & $\begin{array}{l}0.02 \\
0.02 \\
0.02 \\
0.02\end{array}$ & $\begin{array}{l}0.48 \\
0.47 \\
0.46 \\
0.42\end{array}$ \\
\hline $\begin{array}{l}\text { AVE. } \\
\text { STD. }\end{array}$ & & & $\begin{array}{r}45.6 \\
0.3\end{array}$ & $\begin{array}{l}4.3 \\
0.1\end{array}$ & $\begin{array}{l}7.9 \\
0.1\end{array}$ & $\begin{array}{l}5.0 \\
0.1\end{array}$ & $\begin{array}{r}13.2 \\
0.2\end{array}$ & $\begin{array}{l}3.1 \\
0.1\end{array}$ & $\begin{array}{r}15.1 \\
0.5\end{array}$ & $\begin{array}{l}3.5 \\
0.1\end{array}$ & $\begin{array}{l}1.4 \\
0.1\end{array}$ & $\begin{array}{l}0.02 \\
0.01\end{array}$ & $\begin{array}{l}0.39 \\
0.06\end{array}$ & \\
\hline
\end{tabular}

TABLE 35. Weight Loss of Canister 10 Samples Under $\mathrm{pH} 4$ Conditions

\begin{tabular}{|c|c|c|c|c|c|c|}
\hline \multirow{3}{*}{\multicolumn{2}{|c|}{$\frac{\text { Core Height }}{\mathrm{m} t}$}} & \multicolumn{4}{|c|}{ wt\% Loss } & \multirow[b]{3}{*}{ Ave. } \\
\hline & & \multirow{2}{*}{$\begin{array}{l} \\
0-7.6 \\
(0-3)\end{array}$} & \multirow{2}{*}{\multicolumn{2}{|c|}{$\begin{array}{c}\text { Core Depth, cm (in.) } \\
7.6-15.2 \\
15.2-22.9\end{array}$}} & \multirow[b]{2}{*}{$\begin{array}{c}22.9-30.5 \\
(9-12)\end{array}$} & \\
\hline & & & & & & \\
\hline 0.30 & 1 & 74.8 & 72.8 & 75.0 & 69.5 & 74.2 \\
\hline 0.61 & 2 & 73.7 & 71.4 & 74.0 & 75.7 & 73.7 \\
\hline 0.91 & 3 & 74.9 & 78.6 & 72.7 & 72.7 & 74.7 \\
\hline AVE. & & 74.5 & 74.3 & 73.9 & 72.6 & \\
\hline
\end{tabular}

Mean Weight Loss $73.8 \pm 2.3$ wt\%

TABLE 36. Weight Loss of Canister 10 Samples Under Soxhlet Conditions

\begin{tabular}{|c|c|c|c|c|c|c|}
\hline \multirow{2}{*}{\multicolumn{2}{|c|}{$\frac{\text { Core Height }}{\mathrm{m}} \mathrm{ft}$}} & \multicolumn{4}{|c|}{ wt\% Loss } & \multirow[b]{2}{*}{ Ave. } \\
\hline & & $\begin{array}{l}0-7.6 \\
(0-3)\end{array}$ & $\begin{array}{c}\text { Core } \\
7.6-15.2 \\
(3-6)\end{array}$ & $\begin{array}{c}h_{2} \mathrm{~cm} \text { (in. } \\
15.2-22.9 \\
(6-9)\end{array}$ & $\begin{array}{c}22.9-30.5 \\
(9-12)\end{array}$ & \\
\hline 0.30 & 1 & 2.16 & 1.91 & 2.19 & 1.76 & 2.00 \\
\hline 0.61 & 2 & 2.14 & 2.37 & 1.70 & 1.94 & 2.04 \\
\hline 0.91 & 3 & 2.29 & 1.97 & 2.21 & 1.94 & 2.10 \\
\hline AVE. & & 2.19 & 2.08 & 2.03 & 1.88 & \\
\hline
\end{tabular}

Mean Weight Loss $2.05 \pm 0.21$ wt\% 


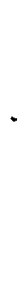




\section{SPRAY CALCINER/CALCINE-FED CERAMIC MELTER}

The Spray Calciner/Calcine-Fed Ceramic Melter (SC/CFCM) system was designed and constructed to demonstrate the operational compatibility of these two solidification process components. The calcination of the liquid waste stream and charging of the calcine and frit into the melter proceeds exactly as described for the SC/ICM process. The melter in this case is a refractorylined joule-heated electric melter.

While some details of design and construction may be unique to the CFCM, the general operating principles are the same as for the LFCM. Alternating current passing through molten glass between two large plate-electrodes maintains the glass temperature at $1100^{\circ}$ to $1200^{\circ} \mathrm{C}$ by joule heating. As the calcine/frit mixture is charged on top of this molten pool, the constituents react, forming a fluid glass. As the glass level in the melter rises, the hydrostatic pressure forces a stream of waste glass out the discharge trough into a receiving canister. Glass flow can be adjusted by tilting the melter assembly. Dierks (1980) provides details of CFCM design and operation.

Three SC/CFCM melting trials involving 10, 25, and 5 continuous days of operation were run processing simulated TDS-211 defense waste glass. The primary objectives were to establish maximum melting rates and gain equipment operating experience. Routine sampling of the liquid waste, frit, and glass product monitored process operation and product consistency. Sample data for each trial will be presented separately.

CFCM-6 (APRIL 1979)

The CFCM had been drained of glass for inspection and modifications prior to the CFCM- 6 melting trial. The primary objectives of this trial were to determine the maximum melting rate of the SC/CFCM system and gain additional equipment operating experience with the TDS-211 defense-waste glass. During 10 continuous days of SC/CFCM operation approximately $11,000 \mathrm{~kg}$ of simulated defense-waste glass were produced, filling seven canisters. Foaming of the 
waste/frit batch was a significant problem limiting maximum production rates. Samples of liquid waste and glass were regularly collected. Physical properties and chemical composition were monitored.

The simulated liquid waste was prepared in $2529-L$ batches to maintain a continuous feed supply to the process. Samples were taken directly from the process feed line immediately upstream of the spray calciner. Operating personnel measured specific gravity and $\mathrm{pH}$ before submitting samples for chemical analysis. Feed data collected during the melting trial is presented in Table 37. Nickel oxide concentrations were not reported since nickel crucibles were used in the analytical procedure.

During the run the liquid feed concentration was increased twice. From the starting level of approximately $46 \mathrm{~g}$ oxide/L solution, the concentration was raised on April 23 to $55 \mathrm{~g} / \mathrm{L}$ and on April 25 to $64 \mathrm{~g} / \mathrm{L}$ to increase the calcine production rate. Figure 13 shows oxide content of the liquid feed during the run. All waste oxide components were consistently below target values during the run due possibly to excessive feed dilution. Since liquid feed rate is a major operating parameter, a lower oxide content translates into a calcine-deficient waste glass. The last concentration increase on April 25, 1979 resulted in significantly greater feed variations. During this same period from April 26, 1979, $1200 \mathrm{~h}, \mathrm{Na}_{2} 0$ content briefly increased from $<0.2 \mathrm{~g} / \mathrm{L}$ to over $2.5 \mathrm{~g} / \mathrm{L}$. The cause of these disturbances were not identified. The mean specific gravity and $\mathrm{pH}$ of over 100 samples were $1.025 \pm 0.008 \mathrm{~g} / \mathrm{cc}$, and $7.7 \pm$ 0.1 , respectively. Neither property was correlated to either total oxide loading or chemical composition of the feed.

Waste glass grab samples were collected approximately every $250 \mathrm{~kg}$ of glass production. The annealed density, chemical composition and crystallinity were determined for each. Annealed glass density is plotted in Figure 14. After April 22, 1979 Frit 211 replaced Frit 411 as the supplier of glass-forming constituents. This is responsible for the sharp increase in density on April 23, 1979. Most major changes in density can be largely accounted for by 
TABLE 37. Composition of CFCM-6 Liquid Feed Samples, g Oxide/L Feed

\begin{tabular}{|c|c|c|c|c|c|c|c|c|c|c|c|}
\hline \multirow[b]{2}{*}{ Date } & \multirow[b]{2}{*}{ Time } & \multirow[b]{2}{*}{$\mathrm{pH}$} & \multirow{2}{*}{$\begin{array}{c}\text { Specific } \\
\text { Gravity, } \\
\text { g/cc } \\
\end{array}$} & \multicolumn{8}{|c|}{ g 0xide/L Feed } \\
\hline & & & & $\begin{array}{l}\text { Total } \\
\text { Oxides } \\
\end{array}$ & $\stackrel{\mathrm{SiO}}{2}_{2}$ & $\underline{\mathrm{Fe}}_{2} \underline{-}_{3}$ & $\mathrm{Al}_{2} \underline{O}_{3}$ & $\underline{\mathrm{CaO}}$ & $\underline{\mathrm{Na}}_{2} \mathrm{O}$ & $\underline{\mathrm{MnO}}_{2}$ & $\mathrm{NiO}^{(\mathrm{a})}$ \\
\hline PART I & & & & & & & & & & & \\
\hline Defined & --- & $\cdots$ & $\cdots$ & 46.0 & 3.3 & 24.2 & 6.1 & 1.8 & 0.4 & 6.4 & 2.8 \\
\hline $4 / 18$ & $\begin{array}{l}0000 \\
0400 \\
0800 \\
1200 \\
1800 \\
2000 \\
2400\end{array}$ & $\begin{array}{l}7.8 \\
8.0 \\
7.9 \\
7.9 \\
7.8 \\
7.8 \\
7.9\end{array}$ & $\begin{array}{l}1.03 \\
1.02 \\
1.02 \\
1.02 \\
1.03 \\
1.03 \\
1.02\end{array}$ & $\begin{array}{l}36.3 \\
37.2 \\
41.0 \\
39.0 \\
43.4 \\
45.0 \\
39.1\end{array}$ & $\begin{array}{l}2.3 \\
2.5 \\
2.9 \\
1.4 \\
4.4 \\
5.0 \\
2.7\end{array}$ & $\begin{array}{l}16.0 \\
15.8 \\
18.3 \\
18.2 \\
18.3 \\
18.1 \\
17.6\end{array}$ & $\begin{array}{l}6.2 \\
6.1 \\
6.5 \\
6.3 \\
7.1 \\
7.3 \\
6.0\end{array}$ & $\begin{array}{l}1.8 \\
1.8 \\
1.8 \\
1.6 \\
1.8 \\
1.9 \\
1.7\end{array}$ & $\begin{array}{l}0.5 \\
1.0 \\
0.7 \\
0.1 \\
0.3 \\
0.3 \\
0.2\end{array}$ & $\begin{array}{l}6.8 \\
6.8 \\
7.3 \\
7.0 \\
7.1 \\
7.1 \\
6.4\end{array}$ & \\
\hline $4 / 19$ & $\begin{array}{l}0400 \\
1600 \\
2000\end{array}$ & $\begin{array}{l}7.9 \\
7.9 \\
7.8\end{array}$ & $\begin{array}{l}1.02 \\
1.03 \\
1.02\end{array}$ & $\begin{array}{l}38.9 \\
39.5 \\
38.0\end{array}$ & $\begin{array}{l}2.6 \\
2.3 \\
3.2\end{array}$ & $\begin{array}{l}17.5 \\
17.9 \\
17.9\end{array}$ & $\begin{array}{l}6.3 \\
5.9 \\
5.1\end{array}$ & $\begin{array}{l}1.8 \\
1.5 \\
1.6\end{array}$ & $\begin{array}{l}0.2 \\
0.1 \\
0.2\end{array}$ & $\begin{array}{l}6.6 \\
6.6 \\
5.7\end{array}$ & \\
\hline $4 / 20$ & $\begin{array}{l}0000 \\
0420 \\
0800 \\
1200 \\
1600 \\
2000\end{array}$ & $\begin{array}{l}7.9 \\
7.9 \\
7.8 \\
7.7 \\
7.7 \\
7.6\end{array}$ & $\begin{array}{l}1.02 \\
1.02 \\
1.02 \\
1.02 \\
1.02 \\
1.02\end{array}$ & $\begin{array}{l}35.1 \\
38.6 \\
40.2 \\
34.2 \\
35.4 \\
35.6\end{array}$ & $\begin{array}{l}1.2 \\
1.4 \\
2.5 \\
2.2 \\
3.2 \\
3.9\end{array}$ & $\begin{array}{l}17.5 \\
18.4 \\
18.5 \\
15.9 \\
15.6 \\
15.8\end{array}$ & $\begin{array}{l}4.7 \\
5.3 \\
5.6 \\
5.1 \\
5.2 \\
5.1\end{array}$ & $\begin{array}{l}1.4 \\
1.2 \\
1.4 \\
1.2 \\
1.4 \\
1.3\end{array}$ & $\begin{array}{l}0.1 \\
0.1 \\
0.1 \\
0.1 \\
0.2 \\
0.2\end{array}$ & $\begin{array}{l}5.9 \\
6.2 \\
6.3 \\
5.2 \\
5.2 \\
4.7\end{array}$ & \\
\hline $4 / 21$ & $\begin{array}{l}0000 \\
0400 \\
0750 \\
1200 \\
1600 \\
2000\end{array}$ & $\begin{array}{l}7.6 \\
7.6 \\
7.6 \\
7.5 \\
7.7 \\
7.6\end{array}$ & $\begin{array}{l}1.02 \\
1.02 \\
1.02 \\
1.03 \\
1.04 \\
1.03\end{array}$ & $\begin{array}{l}45.6 \\
44.7 \\
42.4 \\
39.6 \\
41.8 \\
40.1\end{array}$ & $\begin{array}{l}4.6 \\
3.7 \\
3.1 \\
1.8 \\
2.3 \\
3.1\end{array}$ & $\begin{array}{l}21.9 \\
22.2 \\
20.6 \\
18.1 \\
19.8 \\
19.2\end{array}$ & $\begin{array}{l}6.2 \\
5.7 \\
5.8 \\
5.5 \\
5.6 \\
5.1\end{array}$ & $\begin{array}{l}1.7 \\
1.6 \\
1.7 \\
1.3 \\
1.4 \\
1.5\end{array}$ & $\begin{array}{l}0.3 \\
0.2 \\
0.2 \\
0.1 \\
0.2 \\
0.2\end{array}$ & $\begin{array}{l}5.6 \\
5.8 \\
6.2 \\
5.6 \\
5.4 \\
5.2\end{array}$ & \\
\hline $4 / 22$ & $\begin{array}{l}0025 \\
0405 \\
0800 \\
1200 \\
1600 \\
2000\end{array}$ & $\begin{array}{l}7.7 \\
7.6 \\
7.7 \\
7.6 \\
7.7 \\
7.6\end{array}$ & $\begin{array}{l}1.02 \\
1.02 \\
1.02 \\
1.03 \\
1.02 \\
1.02\end{array}$ & $\begin{array}{l}42.3 \\
43.5 \\
33.0 \\
44.0 \\
35.0 \\
42.1\end{array}$ & $\begin{array}{l}2.0 \\
2.1 \\
0.6 \\
2.9 \\
0.7 \\
2.5\end{array}$ & $\begin{array}{l}20.8 \\
21.6 \\
15.0 \\
19.0 \\
17.5 \\
19.4\end{array}$ & $\begin{array}{l}5.6 \\
5.8 \\
4.9 \\
5.9 \\
4.3 \\
5.7\end{array}$ & $\begin{array}{l}1.5 \\
1.6 \\
1.4 \\
1.4 \\
1.3 \\
1.4\end{array}$ & $\begin{array}{l}0.1 \\
0.1 \\
0.2 \\
0.2 \\
-.-1 \\
0.1\end{array}$ & $\begin{array}{l}6.0 \\
6.1 \\
4.9 \\
5.6 \\
4.9 \\
6.0\end{array}$ & \\
\hline $4 / 23$ & 0015 & 7.8 & 1.02 & 37.1 & 1.1 & 18.6 & 4.8 & 1.5 & 0.1 & 4.7 & \\
\hline $\begin{array}{l}\text { AVE. } \\
\text { STD. DEV. }\end{array}$ & & $\begin{array}{l}7.7 \\
0.1\end{array}$ & $\begin{array}{l}1.02 \\
0.01\end{array}$ & $\begin{array}{l}39.6 \\
3.5\end{array}$ & $\begin{array}{l}2.6 \\
1.1\end{array}$ & $\begin{array}{r}18.3 \\
1.9\end{array}$ & $\begin{array}{l}5.7 \\
0.7\end{array}$ & $\begin{array}{l}1.5 \\
0.2\end{array}$ & $\begin{array}{l}0.2 \\
0.2\end{array}$ & $\begin{array}{l}6.0 \\
0.8\end{array}$ & \\
\hline
\end{tabular}

(a) Not analyzed 
TABLE 37. (contd)

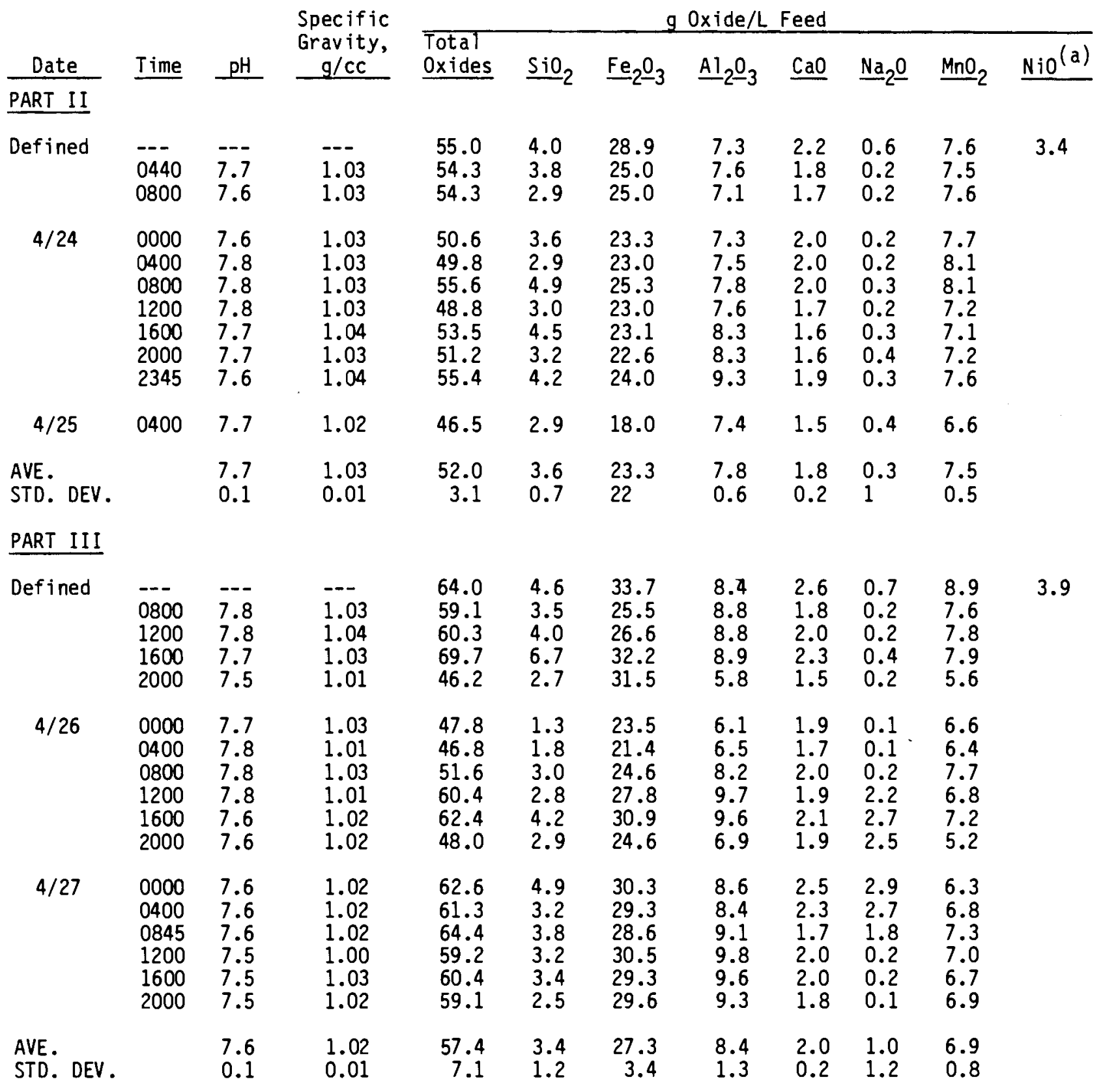

(a) Not analyzed 


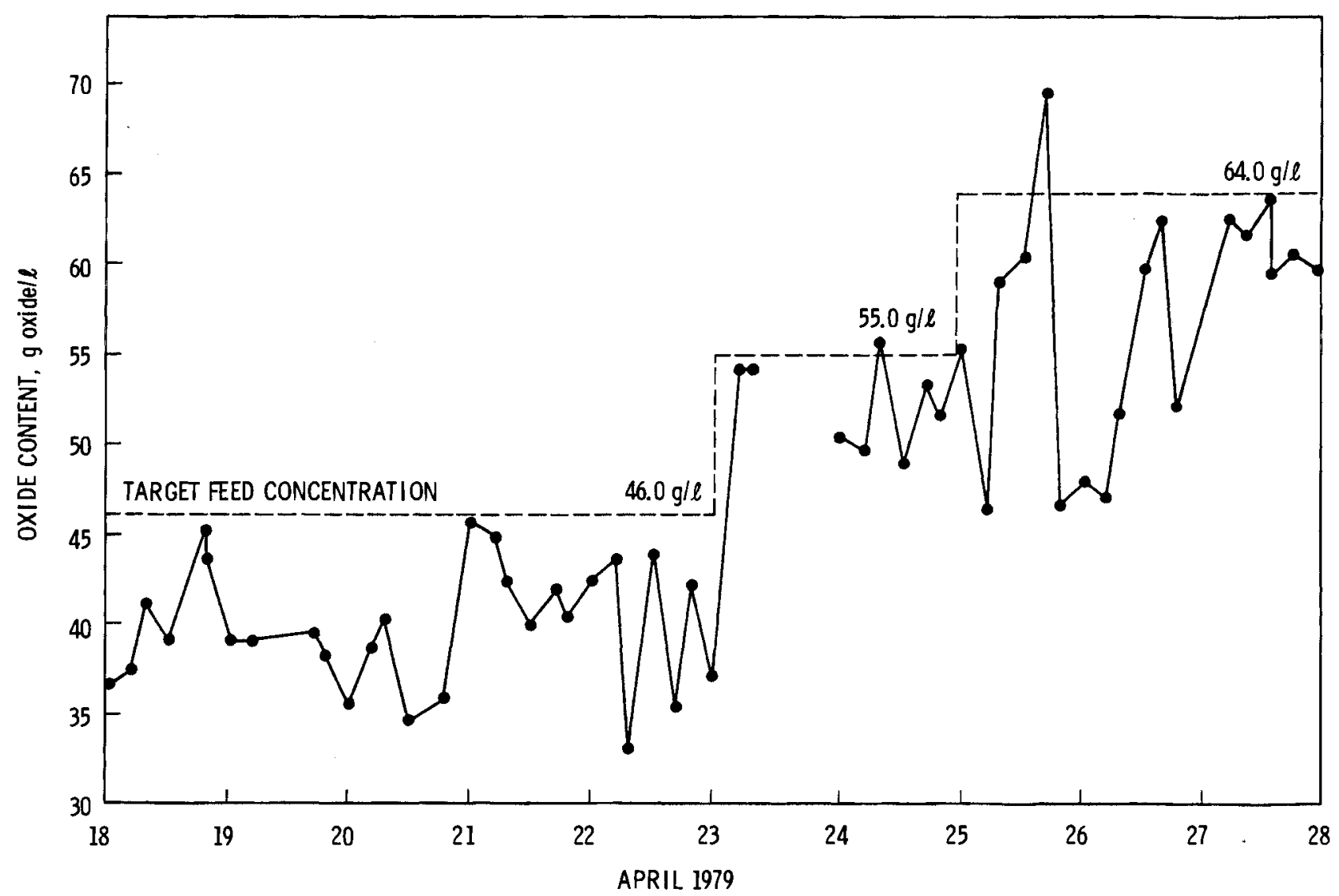

FIGURE 13. Total 0xide Content of CFCM-6 Liquid Feed

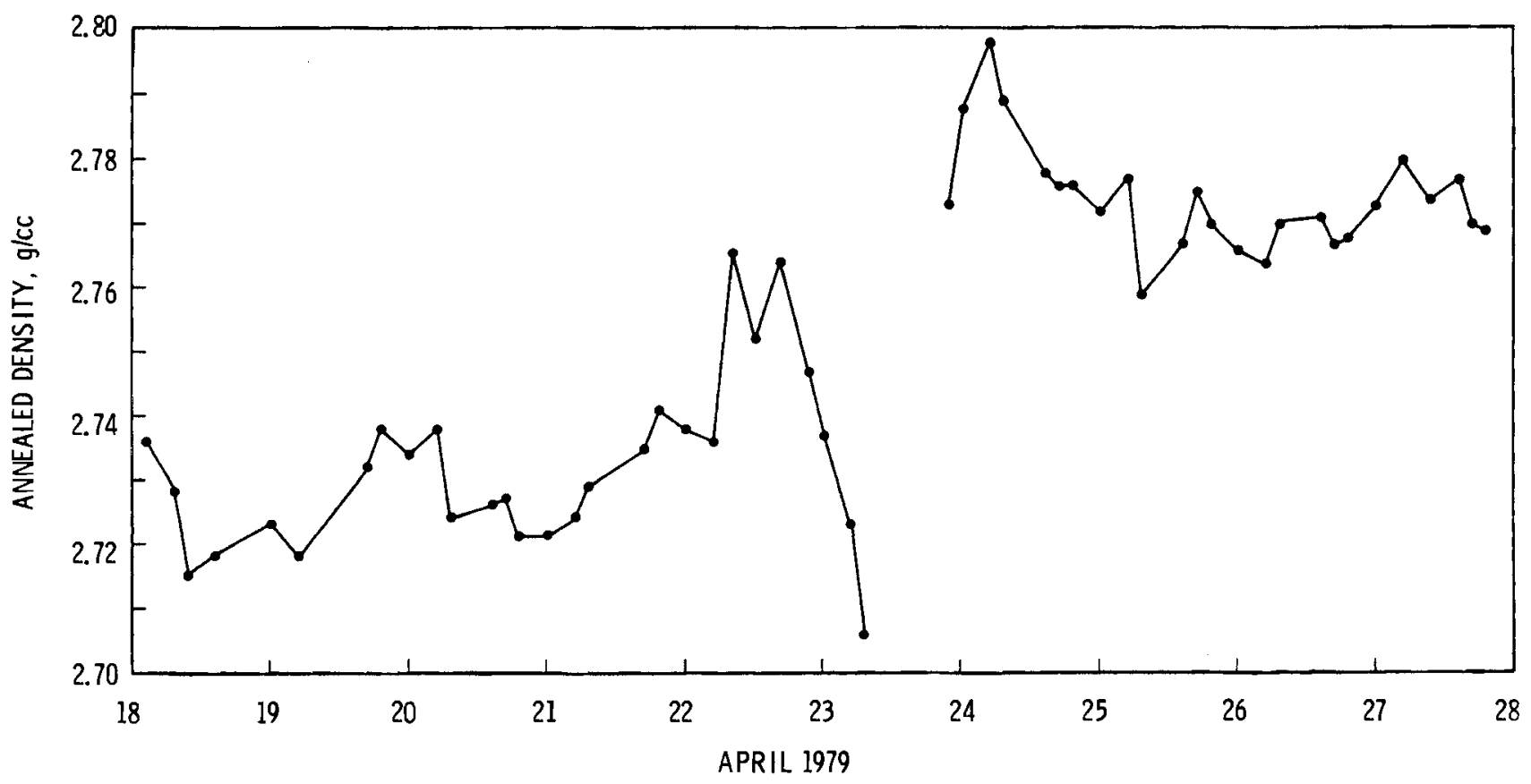

FIGURE 14. Annealed Density of CFCM-6 Grab Samples 
variations in waste loading. The normalized composition and annealed glass density for the grab samples are listed in Table 38. As density increases, the concentration of waste constituents, i.e., $\mathrm{Al}_{2} \mathrm{O}_{3}, \mathrm{Fe}_{2} \mathrm{O}_{3}$ and $\mathrm{MnO}_{2}$, generally increase. This relationship does not hold up as well when waste constituents

TABLE 38. Normalized Composition of CFCM-6 Grab Samples

\begin{tabular}{|c|c|c|c|c|c|c|c|c|c|c|c|}
\hline \multirow[b]{2}{*}{ Date } & \multirow[b]{2}{*}{ Time } & \multirow{2}{*}{$\begin{array}{c}\text { Glass } \\
\text { Produced, } \\
\mathrm{kg} \\
\end{array}$} & \multicolumn{8}{|c|}{ Oxide wt\% } & \multirow{2}{*}{$\begin{array}{l}\text { Density } \\
\mathrm{g} / \mathrm{cc}\end{array}$} \\
\hline & & & $\underline{\mathrm{SO}}_{2}$ & $\underline{A 1}_{2} \underline{0}$ & $\underline{B}_{2} \underline{O}_{2}$ & $\underline{\mathrm{CaO}}$ & $\mathrm{Fe}_{2}{ }_{-3}$ & $\underline{L i}_{2} \underline{0}$ & $\underline{\mathrm{Na}}_{2} \underline{0}$ & $\underline{\mathrm{MnO}} 2$ & \\
\hline $4 / 18$ & $\begin{array}{l}0320 \\
0620 \\
1000 \\
1400 \\
2415\end{array}$ & & $\begin{array}{l}46.8 \\
47.6 \\
47.9 \\
48.4 \\
47.9\end{array}$ & $\begin{array}{l}3.0 \\
2.7 \\
2.7 \\
3.2 \\
3.4\end{array}$ & $\begin{array}{l}8.5 \\
8.6 \\
8.6 \\
8.5 \\
8.4\end{array}$ & $\begin{array}{l}4.6 \\
4.5 \\
4.7 \\
4.6 \\
4.6\end{array}$ & $\begin{array}{l}11.5 \\
10.8 \\
10.2 \\
10.2 \\
10.2\end{array}$ & $\begin{array}{l}9.4 \\
9.7 \\
9.6 \\
9.6 \\
9.9\end{array}$ & $\begin{array}{l}10.6 \\
10.5 \\
10.7 \\
9.6 \\
9.6\end{array}$ & $\begin{array}{l}3.6 \\
3.6 \\
3.6 \\
3.7 \\
3.8\end{array}$ & $\begin{array}{l}2.736 \\
2.728 \\
2.715 \\
2.718 \\
2.723\end{array}$ \\
\hline $4 / 19$ & $\begin{array}{l}0415 \\
1600 \\
2020 \\
2350\end{array}$ & & $\begin{array}{l}48.1 \\
47.5 \\
46.5 \\
46.1\end{array}$ & $\begin{array}{l}3.4 \\
3.5 \\
3.8 \\
3.8\end{array}$ & $\begin{array}{l}8.4 \\
8.3 \\
8.7 \\
8.5\end{array}$ & $\begin{array}{l}4.5 \\
4.6 \\
4.5 \\
4.7\end{array}$ & $\begin{array}{l}10.5 \\
10.6 \\
11.0 \\
11.5\end{array}$ & $\begin{array}{l}9.5 \\
9.4 \\
9.8 \\
9.6\end{array}$ & $\begin{array}{l}9.7 \\
10.2 \\
9.6 \\
9.7\end{array}$ & $\begin{array}{l}3.8 \\
3.9 \\
4.1 \\
4.2\end{array}$ & $\begin{array}{l}2.718 \\
2.732 \\
2.738 \\
2.734\end{array}$ \\
\hline $4 / 20$ & $\begin{array}{l}0405 \\
0800 \\
1130\end{array}$ & 1616 & $\begin{array}{l}46.4 \\
47.1\end{array}$ & $\begin{array}{l}3.7 \\
3.6\end{array}$ & $\begin{array}{l}8.2 \\
8.4\end{array}$ & $\begin{array}{l}4.4 \\
4.5\end{array}$ & $\begin{array}{r}12.5 \\
11.4 \\
\text { CAN }\end{array}$ & $\begin{array}{l}9.1 \\
9.5 \\
\text { TER } 80\end{array}$ & $\begin{array}{l}9.3 \\
9.3 \\
\text { OUT }\end{array}$ & $\begin{array}{l}4.1 \\
4.1\end{array}$ & $\begin{array}{l}2.738 \\
2.724\end{array}$ \\
\hline & $\begin{array}{l}1400 \\
1640 \\
2030\end{array}$ & & $\begin{array}{l}48.2 \\
47.7 \\
47.6\end{array}$ & $\begin{array}{l}3.4 \\
3.4 \\
3.3\end{array}$ & $\begin{array}{l}8.6 \\
8.3 \\
8.6\end{array}$ & $\begin{array}{l}4.5 \\
4.6 \\
4.7\end{array}$ & $\begin{array}{l}10.6 \\
11.3 \\
11.0\end{array}$ & $\begin{array}{l}9.1 \\
9.3 \\
9.3\end{array}$ & $\begin{array}{l}9.5 \\
9.6 \\
9.8\end{array}$ & $\begin{array}{l}3.9 \\
3.8 \\
3.7\end{array}$ & $\begin{array}{l}2.726 \\
2.727 \\
2.721\end{array}$ \\
\hline $4 / 21$ & $\begin{array}{l}0020 \\
0410 \\
0800 \\
1623 \\
2005 \\
2300\end{array}$ & 3184 & $\begin{array}{l}47.7 \\
47.6 \\
47.1 \\
47.5 \\
46.7\end{array}$ & $\begin{array}{l}3.2 \\
3.2 \\
3.3 \\
3.3 \\
3.3\end{array}$ & $\begin{array}{l}8.4 \\
8.7 \\
8.6 \\
8.3 \\
8.1\end{array}$ & $\begin{array}{l}4.3 \\
4.7 \\
4.7 \\
4.6 \\
4.6\end{array}$ & $\begin{array}{r}11.7 \\
11.6 \\
11.4 \\
11.9 \\
12.8 \\
\text { CAN }\end{array}$ & $\begin{array}{l}9.5 \\
9.2 \\
9.2 \\
9.2 \\
9.2 \\
\text { TER } 79\end{array}$ & $\begin{array}{r}9.6 \\
9.7 \\
9.9 \\
9.6 \\
9.5 \\
\text { OUT }\end{array}$ & $\begin{array}{l}3.4 \\
3.6 \\
3.7 \\
3.7 \\
3.7\end{array}$ & $\begin{array}{l}2.721 \\
2.724 \\
2.729 \\
2.735 \\
2.741\end{array}$ \\
\hline $4 / 22$ & $\begin{array}{l}0000 \\
0400 \\
0800 \\
1200 \\
1620 \\
2100\end{array}$ & & $\begin{array}{l}47.4 \\
46.8 \\
45.2 \\
46.5 \\
46.5 \\
46.5\end{array}$ & $\begin{array}{l}3.2 \\
3.2 \\
3.7 \\
3.4 \\
3.4 \\
3.5\end{array}$ & $\begin{array}{l}8.4 \\
8.4 \\
8.5 \\
8.2 \\
8.2 \\
8.1\end{array}$ & $\begin{array}{l}4.5 \\
4.6 \\
4.5 \\
4.5 \\
4.5 \\
4.4\end{array}$ & $\begin{array}{l}11.9 \\
12.6 \\
13.8 \\
13.0 \\
13.1 \\
13.0\end{array}$ & $\begin{array}{l}9.0 \\
9.2 \\
8.8 \\
9.1 \\
7.6 \\
7.6\end{array}$ & $\begin{array}{l}9.7 \\
9.5 \\
9.4 \\
9.5 \\
10.9 \\
11.0\end{array}$ & $\begin{array}{l}3.7 \\
3.6 \\
4.2 \\
3.7 \\
3.8 \\
3.9\end{array}$ & $\begin{array}{l}2.738 \\
2.736 \\
2.767 \\
2.752 \\
2.764 \\
2.747\end{array}$ \\
\hline $4 / 23$ & $\begin{array}{l}0015 \\
0415 \\
0800 \\
1105\end{array}$ & 4754 & $\begin{array}{l}49.2 \\
51.0 \\
51.7\end{array}$ & $\begin{array}{l}3.0 \\
2.8 \\
2.6\end{array}$ & $\begin{array}{l}8.7 \\
9.0 \\
9.2\end{array}$ & $\begin{array}{l}4.3 \\
4.6 \\
4.5\end{array}$ & $\begin{array}{l}10.8 \\
10.4 \\
10.0 \\
\text { CAN }\end{array}$ & $\begin{array}{l}5.4 \\
5.2 \\
4.7 \\
\text { TER } 94\end{array}$ & $\begin{array}{l}13.3 \\
14.6 \\
14.8 \\
\text { OUT }\end{array}$ & $\begin{array}{l}3.3 \\
3.1 \\
2.8\end{array}$ & $\begin{array}{l}2.737 \\
2.723 \\
2.706\end{array}$ \\
\hline & 2200 & & 46.7 & 3.5 & 8.3 & 4.6 & 13.2 & 4.0 & 13.7 & 2.8 & 2.773 \\
\hline
\end{tabular}

(a) NiO not analyzed

(b) Not tabulated 
TABLE 38. (contd)

\begin{tabular}{|c|c|c|c|c|c|c|c|c|c|c|c|}
\hline \multirow[b]{2}{*}{ Date } & \multirow[b]{2}{*}{$\underline{T \text { ime }}$} & \multirow{2}{*}{$\begin{array}{c}\text { Glass } \\
\text { Produced, } \\
\mathrm{kg} \\
\end{array}$} & \multicolumn{8}{|c|}{ 0xide wt\% } & \multirow{2}{*}{$\begin{array}{l}\text { Density } \\
\mathrm{g} / \mathrm{cc}\end{array}$} \\
\hline & & & $\underline{\mathrm{SO}}_{2-}$ & $\mathrm{Al}_{2} 2 \underline{0}$ & $\underline{B}_{2} \underline{O}_{2}$ & CaO & $\mathrm{Fe}_{2} \mathrm{O}_{3}$ & $\underline{L i} \underline{i}_{2} \underline{0}$ & $\underline{\mathrm{Na}}_{2} \underline{0}$ & $\frac{\mathrm{MnO}}{2}$ & \\
\hline $4 / 24$ & $\begin{array}{l}0015 \\
0415 \\
0810 \\
1400 \\
1620 \\
2025 \\
2345\end{array}$ & & $\begin{array}{l}45.8 \\
45.9 \\
45.6 \\
46.4 \\
46.8 \\
47.4 \\
47.2\end{array}$ & $\begin{array}{l}3.7 \\
4.0 \\
3.7 \\
3.5 \\
3.2 \\
3.6 \\
3.7\end{array}$ & $\begin{array}{l}8.0 \\
8.1 \\
8.0 \\
8.0 \\
8.1 \\
8.4 \\
8.1\end{array}$ & $\begin{array}{l}4.6 \\
4.5 \\
4.7 \\
4.6 \\
4.5 \\
4.2 \\
4.5\end{array}$ & $\begin{array}{l}14.0 \\
13.8 \\
14.9 \\
13.6 \\
12.4 \\
12.4 \\
12.6\end{array}$ & $\begin{array}{l}3.9 \\
3.6 \\
3.4 \\
3.4 \\
3.3 \\
3.3 \\
3.4\end{array}$ & $\begin{array}{l}13.6 \\
13.5 \\
13.4 \\
14.5 \\
15.6 \\
14.6 \\
14.6\end{array}$ & $\begin{array}{l}4.2 \\
4.5 \\
4.3 \\
4.0 \\
4.0 \\
4.1 \\
3.9\end{array}$ & $\begin{array}{l}2.788 \\
2.798 \\
2.789 \\
2.778 \\
2.776 \\
2.776 \\
2.772\end{array}$ \\
\hline $4 / 25$ & $\begin{array}{l}0013 \\
0411 \\
0807 \\
1400 \\
1600 \\
2015\end{array}$ & 6516 & $\begin{array}{l}47.2 \\
47.8 \\
47.6 \\
46.9 \\
47.8\end{array}$ & $\begin{array}{l}3.5 \\
3.4 \\
3.7 \\
3.8 \\
3.6\end{array}$ & $\begin{array}{l}8.0 \\
8.1 \\
8.3 \\
8.2 \\
8.4\end{array}$ & $\begin{array}{l}4.7 \\
4.6 \\
4.2 \\
4.4 \\
4.1\end{array}$ & $\begin{array}{c}\text { CANI } \\
12.5 \\
11.7 \\
12.6 \\
14.0 \\
12.9\end{array}$ & $\begin{array}{l}\text { TER } 81 \\
3.3 \\
3.4 \\
3.4 \\
3.3 \\
3.3\end{array}$ & $\begin{array}{l}\text { OUT } \\
15.0 \\
15.4 \\
14.5 \\
13.9 \\
14.1\end{array}$ & $\begin{array}{l}3.8 \\
3.5 \\
3.6 \\
3.5 \\
3.6\end{array}$ & $\begin{array}{l}2.777 \\
2.759 \\
2.767 \\
2.775 \\
2.770\end{array}$ \\
\hline $4 / 26$ & $\begin{array}{l}0000 \\
0150 \\
0410 \\
0800 \\
1440 \\
1600 \\
2000\end{array}$ & 7970 & $\begin{array}{l}48.3 \\
46.7 \\
47.0 \\
40.2 \\
41.5 \\
41.6\end{array}$ & $\begin{array}{l}3.5 \\
3.2 \\
3.6 \\
4.3 \\
4.4 \\
4.5\end{array}$ & $\begin{array}{l}8.7 \\
8.0 \\
8.2 \\
7.7 \\
8.0 \\
8.0\end{array}$ & $\begin{array}{l}4.1 \\
4.6 \\
4.4 \\
5.0 \\
4.7 \\
4.6\end{array}$ & $\begin{array}{c}12.0 \\
\text { CANI } \\
14.6 \\
12.9 \\
16.6 \\
14.2 \\
14.1\end{array}$ & $\begin{array}{l}3.4 \\
\text { TER } 116 \\
3.3 \\
3.4 \\
3.3 \\
3.4 \\
3.4\end{array}$ & $\begin{array}{l}14.5 \\
\text { OUT } \\
14.2 \\
14.8 \\
17.0 \\
18.0 \\
18.0\end{array}$ & $\begin{array}{l}3.6 \\
3.3 \\
3.5 \\
3.7 \\
3.7 \\
3.7\end{array}$ & $\begin{array}{l}2.766 \\
2.764 \\
2.770 \\
2.771 \\
2.767 \\
2.768\end{array}$ \\
\hline $4 / 27$ & $\begin{array}{l}0010 \\
0300 \\
0420 \\
0845 \\
1425 \\
1610 \\
1950\end{array}$ & 10964 & $\begin{array}{l}40.8 \\
40.0 \\
40.1 \\
39.9 \\
38.8 \\
39.3\end{array}$ & $\begin{array}{l}4.2 \\
4.1 \\
4.3 \\
5.0 \\
4.6 \\
4.7\end{array}$ & $\begin{array}{l}8.0 \\
7.7 \\
7.9 \\
7.6 \\
7.4 \\
7.5\end{array}$ & $\begin{array}{l}4.5 \\
5.0 \\
4.9 \\
4.6 \\
4.8 \\
4.6\end{array}$ & $\begin{array}{c}13.5 \\
\text { CANI } \\
14.7 \\
16.0 \\
15.9 \\
14.9 \\
14.8 \\
\text { CANI }\end{array}$ & $\begin{array}{c}3.1 \\
\text { TER } 92 \\
3.0 \\
3.4 \\
4.5 \\
4.0 \\
4.2 \\
\text { TER } 84\end{array}$ & $\begin{array}{l}20.2 \\
\text { OUT } \\
20.1 \\
18.0 \\
17.0 \\
20.1 \\
19.2 \\
\text { OUT }\end{array}$ & $\begin{array}{l}3.6 \\
3.4 \\
3.4 \\
3.6 \\
3.5 \\
3.5\end{array}$ & $\begin{array}{l}2.773 \\
2.780 \\
2.774 \\
2.777 \\
2.770\end{array}$ \\
\hline $\begin{array}{l}\text { RUN A } \\
\text { STD. }\end{array}$ & & & $\begin{array}{r}46.1 \\
2.9\end{array}$ & $\begin{array}{l}3.6 \\
0.5\end{array}$ & $\begin{array}{l}8.3 \\
0.4\end{array}$ & $\begin{array}{l}4.6 \\
0.2\end{array}$ & $\begin{array}{r}12.6 \\
1.6\end{array}$ & (b) & (b) & $\begin{array}{l}3.7 \\
0.3\end{array}$ & $\begin{array}{l}2.751 \\
0.024\end{array}$ \\
\hline $\begin{array}{l}\text { CANI } \\
\text { AVE. } \\
\text { STD. }\end{array}$ & 92 & & $\begin{array}{r}43.0 \\
3.0\end{array}$ & $\begin{array}{l}4.0 \\
0.5\end{array}$ & $\begin{array}{l}8.0 \\
0.2\end{array}$ & $\begin{array}{l}4.6 \\
0.2\end{array}$ & $\begin{array}{r}14.3 \\
1.3\end{array}$ & $\begin{array}{l}3.3 \\
0.2\end{array}$ & $\begin{array}{r}17.0 \\
2.2\end{array}$ & $\begin{array}{l}3.6 \\
0.2\end{array}$ & $\begin{array}{l}2.769 \\
0.003\end{array}$ \\
\hline
\end{tabular}

vary independently as noted after April 25, 1979. Al1 grab samples collected from the pouring spout were amorphous with no detectable amount of crystalline material. 
Only one canister produced during this trial, 92, was core-drilled. Significant changes in glass composition were noted in grab samples taken while canister 92 was being filled (Table 38 ) with both the waste and $\mathrm{Na}_{2} \mathrm{O}$ content increasing. The average composition was, however, close to the defined glass composition. The standard deviations ranged from 5 to $10 \%$ for each constituent. All canister centerline samples contained significant quantities of acmite crystals ( 7 to $25 \mathrm{wt} \%$ ). Figure 15 shows the lathe-shaped acmite crystals in a micrograph of ground glass particles. Although no spinel was detected by $x$-ray diffraction ( $<1$ wt\%), small, bright spinel crystals can be seen in several of the acmite groups. The quantity of crystalline material increased going towards the bottom of the canister. The only wall sample to contain these phases were at the 0.91-m (3-ft) level (Table 39). This variation in crystal content with sample location was not clearly reflected by variations in chemical durability as seen in Tables 40 and 41 . The percent weight loss under pH4 and soxhlet conditions were $80.2 \pm 4.5$ wt\% and $2.90 \pm$ $0.73 w t \%$, respectively.

\section{CFCM-7 (JUNE 1979)}

Melting trial CFCM-7 was basically a continuation of the previous CFCM- 6 trial. During 25 continuous days of operation, 18 canisters $0.61 \mathrm{~m}(2 \mathrm{ft})$ and $0.91 \mathrm{~m}(3 \mathrm{ft}$ ) in dia were filled with approximately $35,000 \mathrm{~kg}$ of simulated defense waste glass. The maximum sustained production rate was $85 \mathrm{~kg} / \mathrm{h}$ $\left(112 \mathrm{~kg} / \mathrm{h} / \mathrm{m}^{2}\right)$. A more typical rate was $70 \mathrm{~kg} / \mathrm{h}\left(92 \mathrm{~kg} / \mathrm{h} / \mathrm{m}^{2}\right)$. The liquid feed was concentrated to $130 \%$ of the defined flowsheet concentration to increase calciner capacity, which was reduced by the failure of some calciner heating elements. Liquid feed and glass samples were collected approximately every $250 \mathrm{~kg}$ of glass production using the same technique as described for CFCM- 6 . The $\mathrm{pH}$, specific gravity and oxide content were measured for each liquid feed sample. The annealed density of each glass sample was measured. The chemical composition of feed and glass samples were analyzed approximately every $1000 \mathrm{~kg}$ of glass production.

During the previous CFCM- 6 melting trial a process control computer program was developed. The MOSUM test statistic described by Bauer and Hackl 


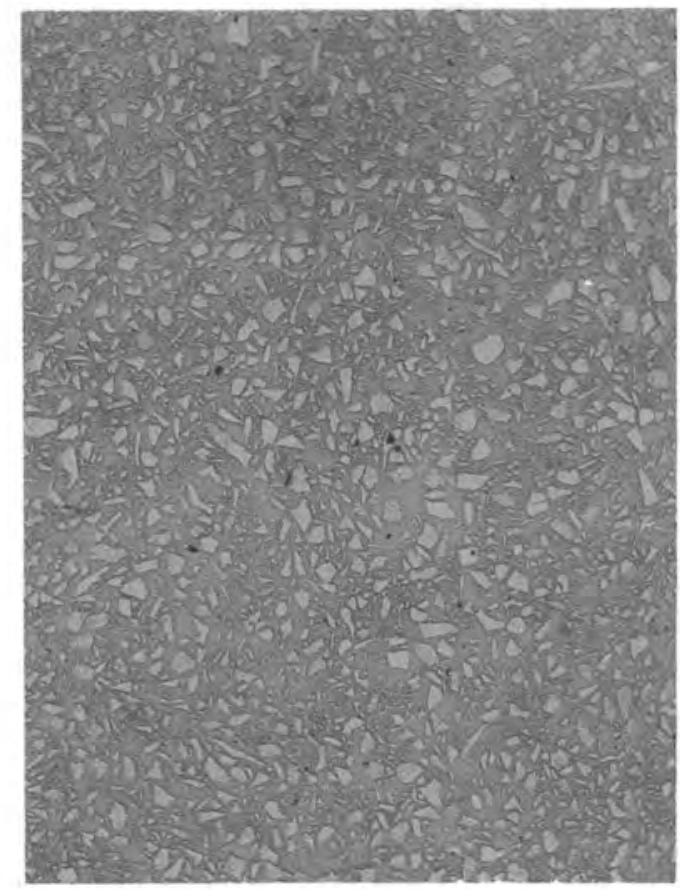

$8.8 \mathrm{X}$

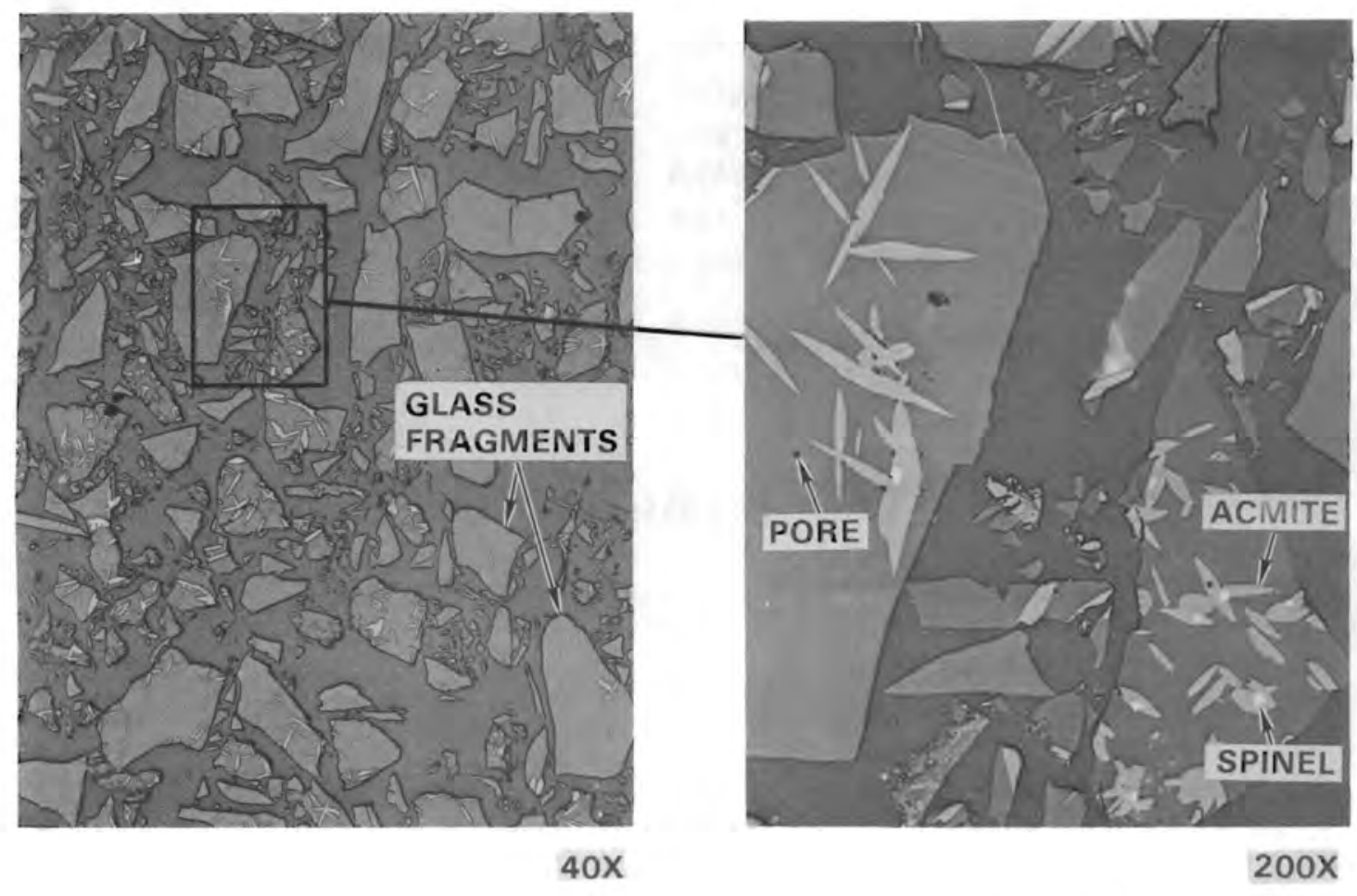

FIGURE 15. Microstructure of Canister 92 Sample [Height $0.30 \mathrm{~m}(1 \mathrm{ft})$; Depth - centerline] 
TABLE 39. Crystalline Content of Canister 92 Samples

\begin{tabular}{|c|c|c|c|}
\hline \multicolumn{2}{|c|}{ Core Height } & \multicolumn{2}{|c|}{ Crystalline Content wt\% } \\
\hline$m$ & $\underline{\mathrm{ft}}$ & Canister Wall & Canister Centerline \\
\hline 0 & 0 & -- & 0 \\
\hline 0.30 & 1 & 0 & 25 wt\% Acmite $(a)$ \\
\hline 0.61 & 2 & --- & --- \\
\hline 0.91 & 3 & 13 wt\% Acmite & 14 wt\% Acmite ${ }^{(a)}$ \\
\hline 1.21 & 4 & --- & --- \\
\hline 1.52 & 5 & 0 & 8 wt\% Acmite \\
\hline
\end{tabular}

(a) acmite $\mathrm{Na}, \mathrm{Fe}, \mathrm{Si}_{2} \mathrm{O}_{6}$

TABLE 40. Weight Loss of Canister 92 Samples Under pH4 Conditions

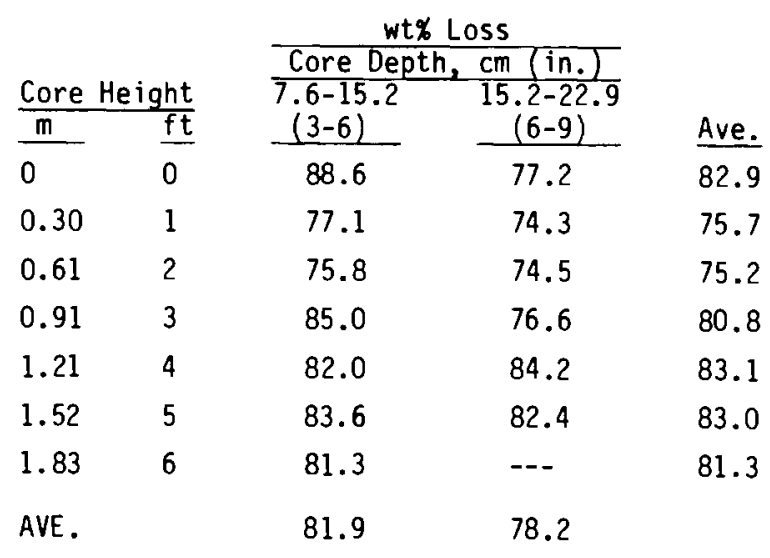

Mean pH4 Weight Loss $80.2 \pm 4.5$ wt\%

TABLE 41. Weight Loss of Canister 92 Samples Under Soxhlet Conditions

\begin{tabular}{|c|c|c|c|c|}
\hline$\frac{\text { Core }}{\mathrm{m}}$ & $\frac{\text { Height }}{f t}$ & 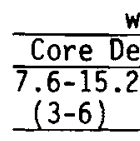 & $\begin{array}{l}\text { oss } \\
\text { cm (in.) } \\
\begin{array}{c}15.2-22.9 \\
(6-9)\end{array} \\
\end{array}$ & Ave. \\
\hline 0 & 0 & 2.32 & 3.19 & 2.74 \\
\hline 0.30 & 1 & 4.51 & 3.55 & 4.03 \\
\hline 0.61 & 2 & 3.58 & 3.36 & 3.47 \\
\hline 0.91 & 3 & 2.22 & 2.48 & 2.35 \\
\hline 1.21 & 4 & 2.31 & 2.43 & 2.37 \\
\hline 1.52 & 5 & 2.31 & 2.51 & 2.41 \\
\hline AVE. & & 2.88 & 2.92 & \\
\hline
\end{tabular}

Mean Soxhlet Weight Loss $2.90 \pm 0.73$ wt\%. 
(1978) indicates when a variable or constituent is beyond its normal range. Prior to April 21, 1979, $\mathrm{MnO}_{2}, \mathrm{CaO}$ and $\mathrm{Na}_{2} \mathrm{O}$ in the liquid feed were outside of control limits. The uncertain sampling error involved with $\mathrm{MnO}_{2}$ and the relatively low levels of $\mathrm{Na}_{2} \mathrm{O}$ did not justify feed modifications for either component. On April 21, 1979 the feed $\mathrm{CaCO}_{3}$ content was increased 15\% to bring $\mathrm{CaO}$ within control limits. A graph of feed $\mathrm{CaO}$ content during the run clearly shows the anticipated response (Figure 16 ). The feed composition during the trial listed in Table 42 was within control limits for all components except $\mathrm{Na}_{2} \mathrm{O}$ which was higher than defined levels. The variation of $\mathrm{Na}_{2} \mathrm{O}, \mathrm{SiO}_{2}$, and $\mathrm{MnO}_{2}$ was approximately $12 \%, 14 \%$, and $39 \%$. All other constituents varied approximately 5\%. As in CFCM-6, no relationship was found between specific gravity or $\mathrm{pH}$ of the feed and its oxide content or composition. The mean specific gravity was $1.041 \pm 0.012 \mathrm{~g} / \mathrm{cc}$.

Grab samples of the glass product collected every $1000 \mathrm{~kg}$ of production were chemically and crystallographically examined. Throughout the trial, all

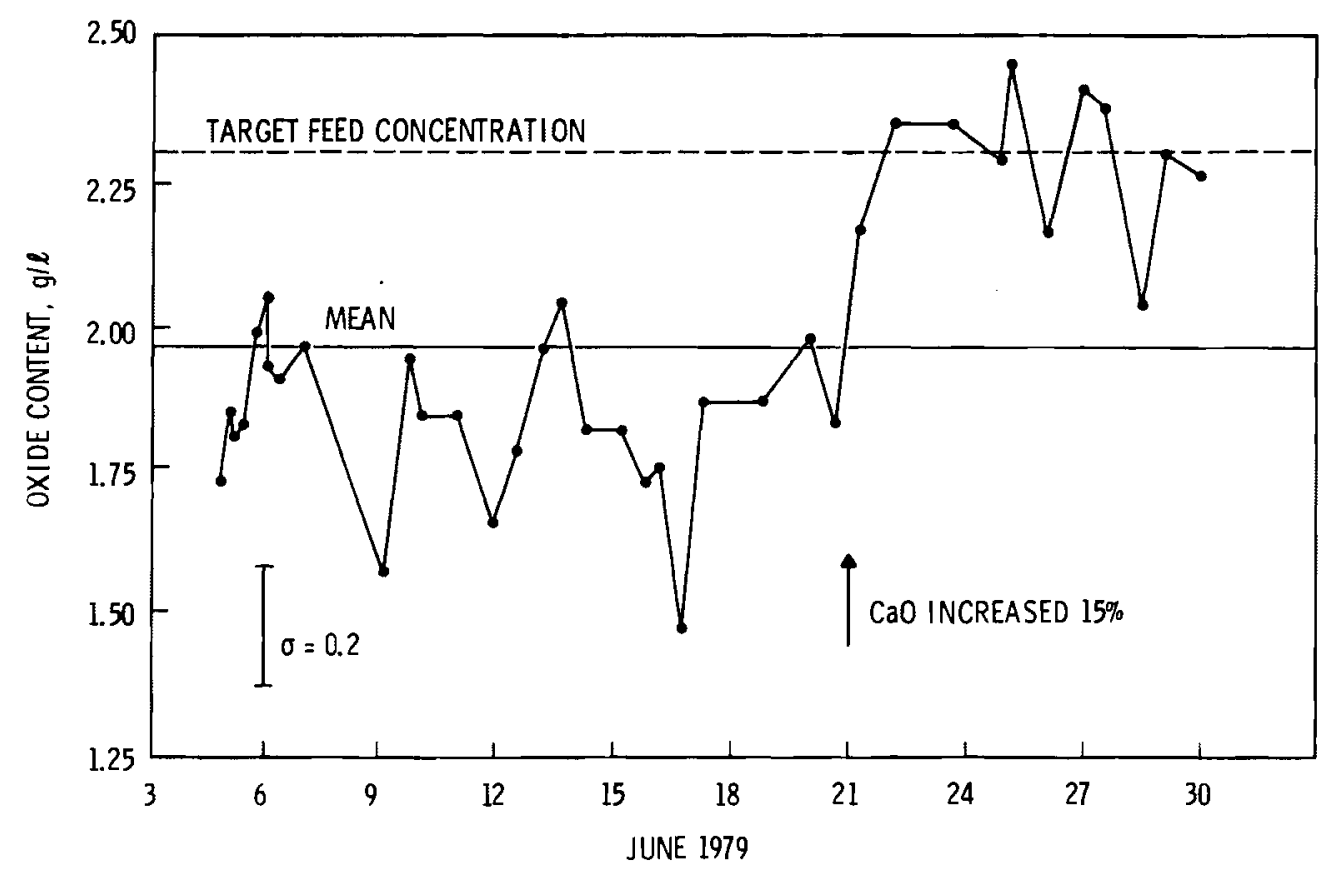

FIGURE 16. CaO Content of CFCM-7 Liquid Feed 


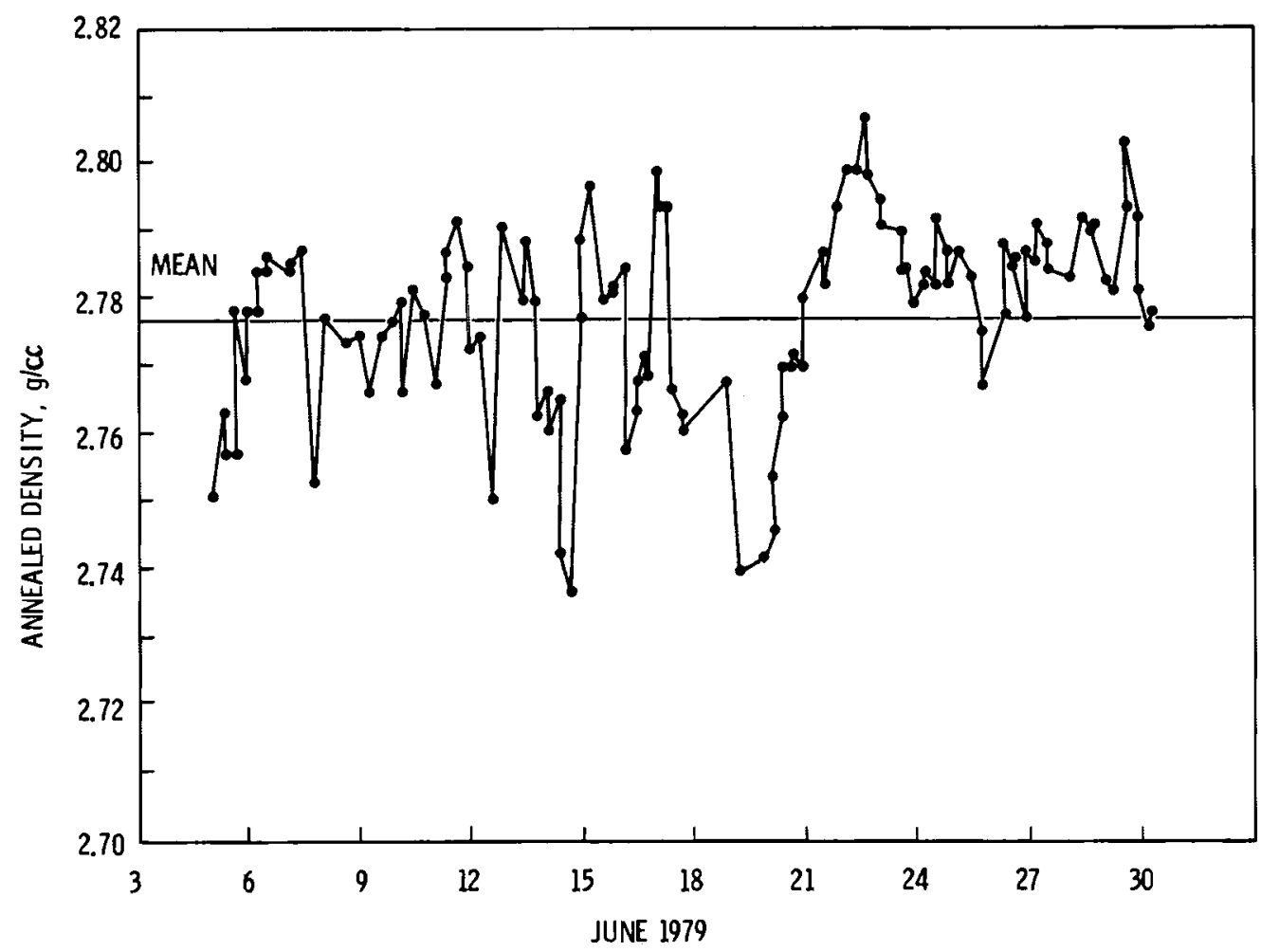

FIGURE 17. Annealed Density CFCM-7 Grab Samples

constituents were with in acceptable composition control limits with the exception of $\mathrm{CaO}$, which was low. The normalized composition of glass samples are listed in Table 43. The annealed density of samples collected during the trial is plotted in Figure 17 and shows that major density changes are superimposed over normal density variation. The correlation coefficients between glass density and composition in Table 44 show a significant positive relationship with major waste constituents such as $\mathrm{Fe}_{2} \mathrm{O}_{3}, \mathrm{MnO}_{2}$ and $\mathrm{NiO}$ and negative relationship with frit constituents $\mathrm{SiO}_{2}, \mathrm{Li}_{2} \mathrm{O}$ and $\mathrm{B}_{2} \mathrm{O}_{3}$. This suggests that a major source of glass compositional variation is the waste/frit ratio delivered to the melter. Additional variation is caused by feed consistency. Although the large variation in feed $\mathrm{MnO}_{2}$ content was largely sampling error, the large variation noted in the glass $\mathrm{MnO}_{2}$ content suggests that $\mathrm{MnO}_{2}$ was not being consistently delivered to the process. 
TABLE 42. Composition of CFCM-7 Liquid Feed Samples

\begin{tabular}{|c|c|c|c|c|c|c|c|c|c|}
\hline \multirow[b]{2}{*}{ Date } & \multirow[b]{2}{*}{ Time } & \multicolumn{8}{|c|}{ g Oxide/L Feed } \\
\hline & & Total & $\underline{\mathrm{SiO}}_{2}$ & $\mathrm{Al}_{2} \underline{0}_{3}$ & $\mathrm{CaO}$ & $\mathrm{Fe}_{2} \underline{O}_{3}$ & $\underline{\mathrm{Na}}_{2} \underline{0}$ & $\underline{\mathrm{MnO}}_{2}$ & $\underline{\mathrm{NiO}}$ \\
\hline Defined & & 64.9 & 4.5 & 8.5 & 2.6 & 34.9 & 1.4 & 9.1 & 4.0 \\
\hline $6 / 5 / 79$ & $\begin{array}{l}0300 \\
0710 \\
1101\end{array}$ & $\begin{array}{l}54.2 \\
56.1 \\
58.7\end{array}$ & $\begin{array}{l}4.8 \\
4.5 \\
5.3\end{array}$ & $\begin{array}{l}8.1 \\
8.0 \\
8.0\end{array}$ & $\begin{array}{l}2.0 \\
2.1 \\
2.1\end{array}$ & $\begin{array}{l}32.0 \\
32.3 \\
34.1\end{array}$ & $\begin{array}{l}2.1 \\
2.4 \\
2.0\end{array}$ & $\begin{array}{l}1.5 \\
2.2 \\
2.3\end{array}$ & $\begin{array}{l}3.5 \\
4.3 \\
4.5\end{array}$ \\
\hline $6 / 6 / 79$ & $\begin{array}{l}0345 \\
0600 \\
0920 \\
1235\end{array}$ & $\begin{array}{l}59.0 \\
61.4 \\
60.2 \\
58.7\end{array}$ & $\begin{array}{l}4.0 \\
4.3 \\
4.8 \\
3.6\end{array}$ & $\begin{array}{l}8.3 \\
9.1 \\
8.5 \\
8.0\end{array}$ & $\begin{array}{l}2.2 \\
2.2 \\
2.1 \\
2.1\end{array}$ & $\begin{array}{l}34.1 \\
36.4 \\
35.0 \\
34.5\end{array}$ & $\begin{array}{l}2.0 \\
2.2 \\
2.4 \\
2.8\end{array}$ & $\begin{array}{l}3.4 \\
1.9 \\
2.4 \\
2.8\end{array}$ & $\begin{array}{l}4.6 \\
4.9 \\
4.7 \\
4.6\end{array}$ \\
\hline $6 / 7 / 79$ & 0200 & 59.3 & 4.2 & 8.6 & 2.2 & 34.6 & 2.4 & 2.2 & 4.7 \\
\hline $6 / 9 / 79$ & $\begin{array}{l}0940 \\
1950\end{array}$ & $\begin{array}{l}58.9 \\
63.3\end{array}$ & $\begin{array}{l}4.4 \\
4.6\end{array}$ & $\begin{array}{l}8.4 \\
9.5\end{array}$ & $\begin{array}{l}1.8 \\
2.2\end{array}$ & $\begin{array}{l}34.9 \\
35.1\end{array}$ & $\begin{array}{l}2.5 \\
2.6\end{array}$ & $\begin{array}{l}2.0 \\
4.2\end{array}$ & $\begin{array}{l}4.5 \\
4.6\end{array}$ \\
\hline $6 / 10 / 79$ & 0800 & 62.9 & 4.6 & 8.9 & 2.1 & 35.9 & 3.0 & 3.4 & 4.5 \\
\hline $6 / 11 / 79$ & $\begin{array}{l}0508 \\
2300\end{array}$ & $\begin{array}{l}61.9 \\
60.4\end{array}$ & $\begin{array}{l}4.6 \\
4.5\end{array}$ & $\begin{array}{l}9.1 \\
8.9\end{array}$ & $\begin{array}{l}2.1 \\
1.9\end{array}$ & $\begin{array}{l}35.5 \\
35.1\end{array}$ & $\begin{array}{l}2.7 \\
2.5\end{array}$ & $\begin{array}{l}3.2 \\
2.6\end{array}$ & $\begin{array}{l}4.3 \\
4.5\end{array}$ \\
\hline $6 / 12 / 79$ & 1530 & 57.6 & 4.0 & 8.1 & 2.0 & 33.2 & 2.8 & 2.5 & 4.3 \\
\hline $6 / 13 / 79$ & $\begin{array}{l}0750 \\
2255\end{array}$ & $\begin{array}{l}64.7 \\
59.7\end{array}$ & $\begin{array}{l}4.7 \\
4.7\end{array}$ & $\begin{array}{l}8.8 \\
9.6\end{array}$ & $\begin{array}{l}2.2 \\
2.3\end{array}$ & $\begin{array}{l}35.5 \\
29.2\end{array}$ & $\begin{array}{l}3.1 \\
2.2\end{array}$ & $\begin{array}{l}5.6 \\
7.0\end{array}$ & $\begin{array}{l}4.4 \\
4.3\end{array}$ \\
\hline $6 / 14 / 79$ & 1320 & 57.6 & 4.0 & 8.1 & 2.0 & 33.2 & 2.8 & 2.5 & 4.2 \\
\hline $6 / 15 / 79$ & $\begin{array}{l}0620 \\
1925\end{array}$ & $\begin{array}{l}53.8 \\
59.3\end{array}$ & $\begin{array}{l}3.2 \\
4.8\end{array}$ & $\begin{array}{l}8.2 \\
8.6\end{array}$ & $\begin{array}{l}2.0 \\
2.0\end{array}$ & $\begin{array}{l}31.1 \\
34.6\end{array}$ & $\begin{array}{l}2.2 \\
2.4\end{array}$ & $\begin{array}{l}2.3 \\
2.2\end{array}$ & $\begin{array}{l}4.4 \\
4.5\end{array}$ \\
\hline $6 / 16 / 79$ & $\begin{array}{l}0800 \\
2035\end{array}$ & $\begin{array}{l}57.2 \\
57.8\end{array}$ & $\begin{array}{l}4.4 \\
4.1\end{array}$ & $\begin{array}{l}8.8 \\
8.9\end{array}$ & $\begin{array}{l}2.0 \\
1.7\end{array}$ & $\begin{array}{l}31.7 \\
31.7\end{array}$ & $\begin{array}{l}2.3 \\
2.2\end{array}$ & $\begin{array}{l}2.8 \\
4.2\end{array}$ & $\begin{array}{l}4.7 \\
4.6\end{array}$ \\
\hline $6 / 17 / 79$ & 1230 & 56.4 & 4.2 & 8.6 & 2.1 & 31.0 & 2.2 & 3.5 & 4.3 \\
\hline $6 / 18 / 79$ & 2010 & 56.3 & 4.2 & 8.4 & 2.1 & 32.6 & 2.4 & 2.0 & 4.4 \\
\hline $6 / 20 / 79$ & $\begin{array}{l}0500 \\
1450\end{array}$ & $\begin{array}{l}53.5 \\
56.4\end{array}$ & $\begin{array}{l}2.2 \\
3.3\end{array}$ & $\begin{array}{l}7.7 \\
8.5\end{array}$ & $\begin{array}{l}2.1 \\
2.0\end{array}$ & $\begin{array}{l}32.1 \\
32.8\end{array}$ & $\begin{array}{l}2.9 \\
2.8\end{array}$ & $\begin{array}{l}1.5 \\
2.1\end{array}$ & $\begin{array}{l}4.6 \\
4.6\end{array}$ \\
\hline $\begin{array}{l}6 / 21 / 79 \\
6 / 22 / 79 \\
6 / 23 / 79 \\
6 / 24 / 79 \\
6 / 25 / 79\end{array}$ & $\begin{array}{l}0830 \\
0500 \\
1330 \\
2100 \\
0345\end{array}$ & $\begin{array}{l}59.0 \\
60.9 \\
59.6 \\
59.1 \\
59.7\end{array}$ & $\begin{array}{l}4.1 \\
4.3 \\
4.4 \\
3.7 \\
4.2\end{array}$ & $\begin{array}{l}8.1 \\
8.5 \\
7.8 \\
7.9 \\
8.4\end{array}$ & $\begin{array}{l}2.4 \\
2.6 \\
2.6 \\
2.5 \\
2.7\end{array}$ & $\begin{array}{l}34.4 \\
33.9 \\
34.5 \\
34.2 \\
33.4\end{array}$ & $\begin{array}{l}2.7 \\
2.6 \\
2.6 \\
2.7 \\
2.6\end{array}$ & $\begin{array}{l}2.4 \\
4.0 \\
2.7 \\
3.5 \\
3.7\end{array}$ & $\begin{array}{l}4.4 \\
4.3 \\
4.4 \\
4.3 \\
4.3\end{array}$ \\
\hline $6 / 26 / 79$ & $\begin{array}{l}0430 \\
2320\end{array}$ & $\begin{array}{l}60.6 \\
56.6\end{array}$ & $\begin{array}{l}4.4 \\
3.3\end{array}$ & $\begin{array}{l}8.0 \\
7.4\end{array}$ & $\begin{array}{l}2.4 \\
2.6\end{array}$ & $\begin{array}{l}33.3 \\
33.8\end{array}$ & $\begin{array}{l}3.1 \\
2.0\end{array}$ & $\begin{array}{l}4.3 \\
3.1\end{array}$ & $\begin{array}{l}4.4 \\
4.2\end{array}$ \\
\hline $\begin{array}{l}6 / 27 / 79 \\
6 / 28 / 79 \\
6 / 29 / 79 \\
6 / 30 / 79\end{array}$ & $\begin{array}{l}1315 \\
1130 \\
0525 \\
0104\end{array}$ & $\begin{array}{l}59.2 \\
53.9 \\
58.3 \\
59.8\end{array}$ & $\begin{array}{l}3.9 \\
3.1 \\
3.6 \\
4.1\end{array}$ & $\begin{array}{l}8.5 \\
6.7 \\
8.4 \\
8.2\end{array}$ & $\begin{array}{l}2.6 \\
2.2 \\
2.5 \\
2.5\end{array}$ & $\begin{array}{l}33.5 \\
28.5 \\
31.2 \\
35.3\end{array}$ & $\begin{array}{l}1.8 \\
1.8 \\
2.8 \\
2.5\end{array}$ & $\begin{array}{l}4.4 \\
7.5 \\
2.5 \\
2.6\end{array}$ & $\begin{array}{l}4.2 \\
3.7 \\
4.3 \\
4.2\end{array}$ \\
\hline $\begin{array}{l}\text { AVE. } \\
\text { STD. DEV. }\end{array}$ & & $\begin{array}{r}58.7 \\
2.5\end{array}$ & $\begin{array}{l}4.1 \\
0.6\end{array}$ & $\begin{array}{l}8.4 \\
0.6\end{array}$ & $\begin{array}{l}2.2 \\
0.2\end{array}$ & $\begin{array}{r}33.4 \\
1.8\end{array}$ & $\begin{array}{l}2.5 \\
0.3\end{array}$ & $\begin{array}{l}3.1 \\
1.2\end{array}$ & $\begin{array}{l}4.4 \\
0.2\end{array}$ \\
\hline
\end{tabular}


TABLE 43. Normalized Composition of CFCM-7 Grab Samples

\begin{tabular}{|c|c|c|c|c|c|c|c|c|c|c|}
\hline \multirow[b]{2}{*}{ Date } & \multirow[b]{2}{*}{ Time } & \multicolumn{9}{|c|}{ Oxide wt\% } \\
\hline & & $\mathrm{SiO}_{2}$ & $\underline{A l}_{2} \underline{O}_{3}$ & $\underline{B}_{2} \underline{O}_{3}$ & $\mathrm{CaO}$ & $\underline{\mathrm{Fe}}_{2} \mathrm{O}_{3}$ & $\underline{\mathrm{Li}}_{2} \underline{0}$ & $\mathrm{Na}_{2} \underline{O}$ & $\mathrm{MnO}_{2}$ & NiO \\
\hline Defined & & 44.0 & 3.7 & 8.0 & 5.1 & 14.7 & 3.2 & 15.5 & 3.9 & 1.7 \\
\hline $6 / 4 / 79$ & 2310 & 46.4 & 4.4 & 8.0 & 4.8 & 14.0 & 3.2 & 13.9 & 3.4 & 1.4 \\
\hline $6 / 5 / 79$ & $\begin{array}{l}0300 \\
0710 \\
1100 \\
1510 \\
1840 \\
2325 \\
2330\end{array}$ & $\begin{array}{l}47.6 \\
47.6 \\
45.1 \\
46.1 \\
44.8 \\
45.1\end{array}$ & $\begin{array}{l}3.9 \\
3.8 \\
3.6 \\
3.6 \\
3.9 \\
3.8\end{array}$ & $\begin{array}{l}8.2 \\
8.2 \\
8.0 \\
8.1 \\
8.0 \\
8.0\end{array}$ & $\begin{array}{l}4.7 \\
4.8 \\
4.9 \\
4.9 \\
4.9 \\
4.9 \\
\text { CANIS }\end{array}$ & $\begin{array}{l}13.5 \\
14.0 \\
13.8 \\
13.6 \\
14.3 \\
14.1 \\
\text { TER } 77 \text { OI }\end{array}$ & $\begin{array}{l}3.3 \\
3.1 \\
4.2 \\
2.8 \\
2.8 \\
2.8\end{array}$ & $\begin{array}{l}14.2 \\
14.1 \\
15.8 \\
15.6 \\
15.6 \\
15.4\end{array}$ & $\begin{array}{l}2.6 \\
2.4 \\
2.4 \\
3.0 \\
3.6 \\
3.6\end{array}$ & $\begin{array}{l}1.2 \\
1.3 \\
1.5 \\
1.6 \\
1.6 \\
1.7\end{array}$ \\
\hline $6 / 6 / 79$ & $\begin{array}{l}0350 \\
0600 \\
0920\end{array}$ & $\begin{array}{l}44.7 \\
44.5 \\
44.5\end{array}$ & $\begin{array}{l}3.8 \\
3.9 \\
3.7\end{array}$ & $\begin{array}{l}7.9 \\
7.9 \\
7.8\end{array}$ & $\begin{array}{l}4.8 \\
4.9 \\
4.8\end{array}$ & $\begin{array}{l}13.9 \\
14.1 \\
14.8\end{array}$ & $\begin{array}{l}3.1 \\
2.7 \\
2.7\end{array}$ & $\begin{array}{l}15.5 \\
15.6 \\
15.4\end{array}$ & $\begin{array}{l}3.9 \\
4.0 \\
3.7\end{array}$ & $\begin{array}{l}1.7 \\
1.7 \\
1.9\end{array}$ \\
\hline \multirow[t]{2}{*}{$6 / 7 / 79$} & $\begin{array}{l}0200 \\
0845\end{array}$ & 44.8 & 3.6 & 7.9 & \multirow{2}{*}{\multicolumn{2}{|c|}{$\begin{array}{lcl}4.8 & 13.9 \\
\text { CANISTER } 83 & 0 \\
4.8 & 13.3\end{array}$}} & \multirow{2}{*}{$\begin{array}{l}3.0 \\
\text { OUT } \\
3.1\end{array}$} & 15.1 & 4.4 & 1.9 \\
\hline & 2000 & 45.2 & 3.5 & 8.1 & & & & 15.6 & 4.0 & 1.7 \\
\hline $6 / 9 / 79$ & $\begin{array}{l}0940 \\
1950\end{array}$ & $\begin{array}{l}45.8 \\
45.4\end{array}$ & $\begin{array}{l}3.5 \\
3.7\end{array}$ & $\begin{array}{l}8.1 \\
8.0\end{array}$ & $\begin{array}{l}4.6 \\
4.5\end{array}$ & $\begin{array}{l}13.8 \\
14.2\end{array}$ & $\begin{array}{l}3.3 \\
3.4\end{array}$ & $\begin{array}{l}15.4 \\
15.1\end{array}$ & $\begin{array}{l}3.1 \\
3.3\end{array}$ & $\begin{array}{l}1.7 \\
1.8\end{array}$ \\
\hline $6 / 10 / 79$ & $\begin{array}{l}0800 \\
1355\end{array}$ & 46.0 & 3.6 & 8.0 & $\begin{array}{l}4.6 \\
\text { CANIS }\end{array}$ & $\begin{array}{l}13.3 \\
\text { TER } 700\end{array}$ & 3.4 & 15.6 & 3.2 & 1.6 \\
\hline \multirow[t]{2}{*}{$6 / 11 / 79$} & $\begin{array}{l}0510 \\
1630\end{array}$ & 45.9 & 3.6 & 8.1 & \multirow{2}{*}{\multicolumn{3}{|c|}{$\begin{array}{lcc}4.6 & 12.9 & 3.4 \\
\text { CANI STER } & 200 & \text { OUTं } \\
4.4 & 14.4 & 3.2\end{array}$}} & 15.2 & 4.1 & 15 \\
\hline & 2300 & 44.6 & 3.8 & 7.7 & & & & 16.1 & 3.5 & 1.8 \\
\hline $6 / 12 / 79$ & $\begin{array}{l}1530 \\
1830\end{array}$ & 46.8 & 3.3 & 8.1 & $\begin{array}{l}4.7 \\
\text { CANIS }\end{array}$ & $\begin{array}{l}12.7 \\
\text { TER } 120\end{array}$ & ${ }_{\text {OUT }}^{3.3}$ & 15.4 & 3.4 & 1.5 \\
\hline \multirow[t]{2}{*}{$6 / 13 / 79$} & $\begin{array}{l}0750 \\
1810\end{array}$ & 44.9 & 3.8 & 7.8 & \multirow{2}{*}{\multicolumn{3}{|c|}{$\begin{array}{lcc}4.6 & 14.7 & 3.2 \\
\text { CANISTER } & 109-\mathrm{L} & \text { OUT } \\
4.6 & 13.2 & 3.3\end{array}$}} & 15.2 & 3.3 & 1.8 \\
\hline & 2255 & 45.6 & 3.8 & 7.9 & & & & 15.7 & 3.8 & 1.6 \\
\hline $6 / 14 / 79$ & $\begin{array}{l}1320 \\
1620\end{array}$ & 46.9 & 3.5 & 8.2 & $\begin{array}{l}4.5 \\
\text { CANI }\end{array}$ & $\begin{array}{l}11.6 \\
\text { TER } 110-\end{array}$ & $\begin{array}{l}3.6 \\
\text { L OUT }\end{array}$ & 16.0 & 3.4 & 1.5 \\
\hline \multirow[t]{2}{*}{$6 / 15 / 79$} & $\begin{array}{l}0015 \\
1615\end{array}$ & 43.5 & 4.3 & 7.7 & \multirow{2}{*}{\multicolumn{2}{|c|}{$\begin{array}{lc}4.4 & 14.2 \\
\text { CANISTER } 85 \\
4.4 & 14.1\end{array}$}} & \multirow{2}{*}{$\begin{array}{l}3.4 \\
\text { OUT } \\
3.4\end{array}$} & 15.4 & 4.3 & 1.9 \\
\hline & 1975 & 44.7 & 3.9 & 7.9 & & & & 15.2 & 3.6 & 1.8 \\
\hline
\end{tabular}


TABLE 43. (contd)

Oxide wt\%

\begin{tabular}{|c|c|c|c|c|c|c|c|c|c|}
\hline Date & ime & $\mathrm{SiO}_{2}$ & $A l_{2}-{ }_{3}$ & $\underline{B}_{2} \underline{O}_{3}$ & $\mathrm{CaO}$ & $\mathrm{Fe}_{2}-{ }_{3}$ & $\underline{\mathrm{Li}}_{2} \underline{\underline{0}}$ & $\mathrm{Na}_{2} \underline{O}$ & $\mathrm{MnO}_{2}$ \\
\hline $6 / 16$ & $\begin{array}{l}080 \\
20\end{array}$ & $\begin{array}{l}45.7 \\
44.7\end{array}$ & $\begin{array}{l}3.7 \\
3.9\end{array}$ & $\begin{array}{l}8.1 \\
8.0\end{array}$ & $\begin{array}{l}4.4 \\
4.2\end{array}$ & $\begin{array}{l}12.4 \\
13.0\end{array}$ & $\begin{array}{l}3.5 \\
3.6\end{array}$ & $\begin{array}{l}15.6 \\
15.8\end{array}$ & $\begin{array}{l}4.1 \\
3.9\end{array}$ \\
\hline
\end{tabular}

$6 / 17 / 790730 \quad$ CANISTER 201 OUT

$\begin{array}{llllllllll}1230 & 44.8 & 3.9 & 8.0 & 4.4 & 13.2 & 3.5 & 16.0 & 3.8 & 1.8\end{array}$

$\begin{array}{lllllllllll}6 / 18 / 79 & 2010 & 45.1 & 4.0 & 7.9 & 4.6 & 13.2 & 3.2 & 15.4 & 4.1 & 1.8\end{array}$

$6 / 19 / 790635 \quad$ CANISTER 118 OUT

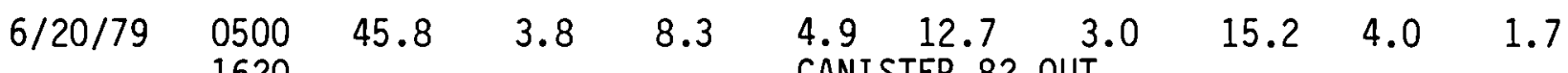

$\begin{array}{lllllllllll}6 / 21 / 79 & 0500 & 44.0 & 4.0 & 7.9 & 4.8 & 14.7 & 3.0 & 14.8 & 4.3 & 1.9\end{array}$

6/22/79 $2210 \quad$ CANISTER 205 OUT

$\begin{array}{lllllllllll}6 / 23 / 79 & 0135 & 44.2 & 3.8 & 7.9 & 4.9 & 15.2 & 2.9 & 14.7 & 3.9 & 1.9\end{array}$

$\begin{array}{llllllllll}0440 & 44.0 & 3.8 & 8.0 & 5.0 & 15.6 & 2.8 & 14.5 & 3.7 & 2.0\end{array}$

$\begin{array}{llllllllll}2015 & 44.4 & 3.7 & 8.0 & 4.9 & 15.1 & 2.9 & 14.5 & 3.8 & 1.9\end{array}$

2330

$\begin{array}{lllllllllll}6 / 24 / 79 & 1310 & 44.3 & 3.8 & 8.0 & 4.8 & 14.8 & 3.0 & 14.7 & 4.2 & 1.8\end{array}$ $\begin{array}{llllllllll}2310 & 44.1 & 3.9 & 7.9 & 4.8 & 14.9 & 3.0 & 14.8 & 4.0 & 1.8\end{array}$ 2330 CANISTER 207 OUT

$\begin{array}{lllllllllll}6 / 25 / 79 & 0050 & 44.3 & 3.8 & 7.9 & 4.7 & 14.6 & 3.1 & 15.1 & 3.9 & 1.8\end{array}$ $\begin{array}{llllllllll}2350 & 45.1 & 3.6 & 8.1 & 4.9 & 13.6 & 3.0 & 15.2 & 4.2 & 1.7\end{array}$

$6 / 26 / 790035 \quad$ CANISTER 211 OUT

$\begin{array}{llllllllll}2005 & 45.3 & 3.6 & 8.0 & 5.1 & 14.2 & 2.7 & 14.5 & 4.2 & 1.8\end{array}$

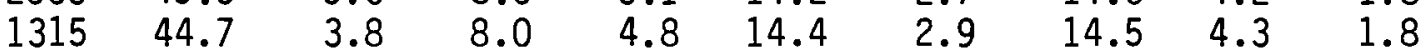

$6 / 27 / 79 \quad 2027$

CANISTER 204 OUT

$\begin{array}{lllllllllll}6 / 28 / 79 & 1130 & 45.3 & 3.7 & 7.9 & 4.8 & 14.6 & 2.9 & 15.1 & 4.1 & 1.8\end{array}$ $\begin{array}{lllllllllll}6 / 29 / 79 & 0600 & 44.8 & 3.9 & 7.7 & 5.0 & 14.4 & 3.0 & 15.1 & 3.7 & 1.8\end{array}$ $\begin{array}{lllllllllll}6 / 30 / 79 & 0100 & 44.5 & 3.6 & 7.8 & 4.8 & 14.2 & 3.1 & 15.3 & 4.2 & 1.8\end{array}$ $\begin{array}{llllllllll}\text { AVE } & 44.6 & 3.8 & 7.9 & 4.7 & 13.8 & 3.1 & 15.0 & 3.7 & 1.7\end{array}$ $\begin{array}{llllllllll}\text { STD. DEV. } & 1.0 & 0.2 & 0.1 & 0.2 & 0.9 & 0.3 & 0.5 & 0.5 & 0.2\end{array}$ 
TABLE 44. Correlation Coefficients of Glass Density and Composition

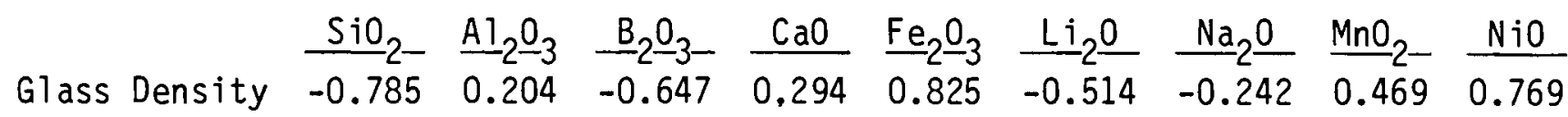

Foaming of the waste/frit batch in the melter was a significant problem during the trial as in CFCM-6. In only one instance on June 7, 1979 did foaming alter waste glass properties by carrying unreacted calcine out of the melter by the glass stream. Crystalline material was detected in only one of the glass samples. In glass samples prior to June 22, 1979 small spheres less than $1 \mathrm{~mm}$ in dia containing primarily $\mathrm{Na}, \mathrm{S}$ and $\mathrm{Cl}$ were occasionally found. The glass during this period contained 0.14 wt\% $\mathrm{Cl}$. This high amount of $\mathrm{Cl}^{-}$was added as an unintentional impurity in one of the ferric hydroxide sources used. Before changing ferric hydroxide suppliers on June 20,1979, liquid feed samples contained $2.37 \mathrm{~g} / \mathrm{L}$ chlorine while subsequent samples contained $0.37 \mathrm{~g} / \mathrm{L}$. The chlorine analyzed in the glass represented less than $20 \%$ of that added in the feed. The anticipated $\mathrm{Cl}^{-}$content of the TDS waste feed is $0.68 \mathrm{~g} / \mathrm{L}$.

One 0.91-m (3-ft)-dia canister of glass, 204, was sampled by core-dri11ing. The normalized composition of these samples is listed in Table 45. Table 46 shows no consistent variation in composition with sample location within the canister. The average glass composition was very close to the defined values with small standard deviations. Although all grab samples were amorphous, nearly all canister samples contained 5 to 20 wt\% acmite (Table 47). Photomicrographs of two samples (Figures 18 and 19) show that the acmite crystals have resulted in extensive microcracking. Small, bright spinel crystals can be seen scattered throughout the sample. Although chemical analysis does not suggest product inhomogeneity, the nonuniform distribution of crystals indicate that localized compositional variations exist. The average sample weight losses under soxhlet and $\mathrm{pH} 4$ conditions were $3.56 \pm 1.58 \mathrm{wt} \%$ and $74.8 \pm$ $9.8 \mathrm{wt} \%$, respectively (Tables 48 and 49 ). The cause of the high variation in weight loss for the relatively homogeneous samples is not known. 
TABLE 45. Normalized Composition of Canister 204 Samples

\begin{tabular}{|c|c|c|c|c|c|c|c|c|c|c|c|c|}
\hline \multicolumn{2}{|c|}{$\begin{array}{l}\text { Core } \\
\text { Height }\end{array}$} & \multicolumn{2}{|c|}{ Core Depth } & \multicolumn{9}{|c|}{ Oxide wt\% } \\
\hline $\mathrm{m}$ & $\overline{f t}$ & $\mathrm{~cm}$ & in. & $\underline{\mathrm{SiO}}_{2}$ & $\mathrm{~A}_{2}{ }_{2}{ }_{-}$ & $\underline{B}_{2}{ }_{-3}$ & $\mathrm{CaO}$ & $\mathrm{Fe}_{2}{ }_{-3}$ & $\underline{L i}_{2} \underline{0}$ & $\underline{\mathrm{Na}}_{2} \underline{0}$ & $\mathrm{MnO}_{2}$ & $\underline{\mathrm{NiO}}$ \\
\hline \multicolumn{2}{|c|}{ Defined } & & & 44.0 & 3.6 & 8.0 & 5.0 & 14.0 & 3.2 & 16.0 & 3.9 & 1.7 \\
\hline 0 & 0 & $\begin{array}{l}0-11.4 \\
11.4-22.9 \\
22.9-34.3 \\
34.3-45.7\end{array}$ & $\begin{array}{l}0-4.5 \\
4.5-9.0 \\
9.0-13.5 \\
13.5-18.0\end{array}$ & $\begin{array}{l}46.2 \\
45.7 \\
45.5 \\
45.5\end{array}$ & $\begin{array}{l}3.5 \\
3.7 \\
3.7 \\
3.7\end{array}$ & $\begin{array}{l}8.2 \\
8.0 \\
7.8 \\
7.8\end{array}$ & $\begin{array}{l}5.0 \\
4.9 \\
5.0 \\
5.1\end{array}$ & $\begin{array}{l}12.6 \\
12.9 \\
13.3 \\
13.7\end{array}$ & $\begin{array}{l}3.3 \\
3.3 \\
3.3 \\
3.2\end{array}$ & $\begin{array}{l}15.4 \\
15.4 \\
15.4 \\
14.8\end{array}$ & $\begin{array}{l}3.5 \\
3.7 \\
3.8 \\
3.9\end{array}$ & $\begin{array}{l}1.6 \\
1.7 \\
1.5 \\
1.7\end{array}$ \\
\hline 0.30 & 1 & $\begin{array}{l}0-11.4 \\
11.4-22.9 \\
22.9-34.3\end{array}$ & $\begin{array}{l}0-4.5 \\
4.5-9.0 \\
9.0-13.5\end{array}$ & $\begin{array}{l}46.1 \\
45.8 \\
45.7\end{array}$ & $\begin{array}{l}3.7 \\
3.6 \\
3.6\end{array}$ & $\begin{array}{l}8.1 \\
7.9 \\
7.9\end{array}$ & $\begin{array}{l}5.2 \\
5.1 \\
5.0\end{array}$ & $\begin{array}{l}13.6 \\
13.4 \\
13.3\end{array}$ & $\begin{array}{l}3.1 \\
3.1 \\
3.2\end{array}$ & $\begin{array}{l}14.3 \\
15.0 \\
15.1\end{array}$ & $\begin{array}{l}4.0 \\
3.9 \\
3.9\end{array}$ & $\begin{array}{l}1.4 \\
1.6 \\
1.6\end{array}$ \\
\hline 0.61 & 2 & $\begin{array}{l}0-11.4 \\
11.4-22.9 \\
22.9-34.3 \\
34.3-45.7\end{array}$ & $\begin{array}{l}0-4.5 \\
4.5-9.0 \\
9.0-13.5 \\
13.5-18.0\end{array}$ & $\begin{array}{l}45.4 \\
45.4 \\
45.8 \\
45.7\end{array}$ & $\begin{array}{l}3.8 \\
3.7 \\
3.7 \\
3.7\end{array}$ & $\begin{array}{l}8.0 \\
7.9 \\
8.0 \\
8.0\end{array}$ & $\begin{array}{l}5.0 \\
5.0 \\
5.1 \\
5.1\end{array}$ & $\begin{array}{l}13.6 \\
13.0 \\
13.6 \\
13.8\end{array}$ & $\begin{array}{l}3.1 \\
3.3 \\
3.1 \\
3.1\end{array}$ & $\begin{array}{l}14.7 \\
15.8 \\
14.8 \\
14.9\end{array}$ & $\begin{array}{l}4.0 \\
3.9 \\
4.0 \\
3.9\end{array}$ & $\begin{array}{l}1.7 \\
1.4 \\
1.2 \\
1.1\end{array}$ \\
\hline 0.91 & 3 & $\begin{array}{l}0-11.4 \\
11.4-22.9 \\
22.9-34.3 \\
34.3-45.7\end{array}$ & $\begin{array}{l}0-4.5 \\
4.5-9.0 \\
9.0-13.5 \\
13.5-18.0\end{array}$ & $\begin{array}{l}45.4 \\
45.2 \\
45.6 \\
45.5\end{array}$ & $\begin{array}{l}3.7 \\
3.7 \\
3.8 \\
3.7\end{array}$ & $\begin{array}{l}8.0 \\
8.0 \\
8.1 \\
8.1\end{array}$ & $\begin{array}{l}4.6 \\
5.0 \\
5.2 \\
5.1\end{array}$ & $\begin{array}{l}13.9 \\
13.1 \\
13.2 \\
14.0\end{array}$ & $\begin{array}{l}3.1 \\
3.3 \\
3.2 \\
3.1\end{array}$ & $\begin{array}{l}14.8 \\
15.9 \\
15.2 \\
14.9\end{array}$ & $\begin{array}{l}4.0 \\
3.8 \\
4.0 \\
4.0\end{array}$ & $\begin{array}{l}1.7 \\
1.4 \\
1.3 \\
1.1\end{array}$ \\
\hline 1.21 & 4 & $\begin{array}{l}0-11.4 \\
11.4-22.9 \\
22.9-34.3 \\
34.3-45.7\end{array}$ & $\begin{array}{l}0-4.5 \\
4.5-9.0 \\
9.0-13.5 \\
13.5-18.0\end{array}$ & $\begin{array}{l}44.8 \\
44.8 \\
45.1 \\
44.9\end{array}$ & $\begin{array}{l}3.8 \\
3.8 \\
3.8 \\
3.9\end{array}$ & $\begin{array}{l}7.9 \\
8.0 \\
7.9 \\
8.1\end{array}$ & $\begin{array}{l}5.0 \\
5.0 \\
5.1 \\
5.2\end{array}$ & $\begin{array}{l}13.9 \\
14.0 \\
14.3 \\
14.2\end{array}$ & $\begin{array}{l}3.1 \\
3.1 \\
3.2 \\
3.1\end{array}$ & $\begin{array}{l}15.1 \\
14.7 \\
15.0 \\
14.0\end{array}$ & $\begin{array}{l}4.0 \\
4.2 \\
4.0 \\
4.1\end{array}$ & $\begin{array}{l}1.7 \\
1.7 \\
1.1 \\
1.9\end{array}$ \\
\hline 1.52 & 5 & $\begin{array}{l}0-11.4 \\
11.4-22.9 \\
22.9-34.3\end{array}$ & $\begin{array}{l}0-4.5 \\
4.5-9.0 \\
9.0-13.5\end{array}$ & $\begin{array}{l}45.4 \\
45.5 \\
45.8\end{array}$ & $\begin{array}{l}3.7 \\
3.7 \\
3.9\end{array}$ & $\begin{array}{l}8.0 \\
7.9 \\
8.1\end{array}$ & $\begin{array}{l}5.1 \\
5.1 \\
4.9\end{array}$ & $\begin{array}{l}13.9 \\
13.8 \\
12.5\end{array}$ & $\begin{array}{l}3.1 \\
3.2 \\
3.4\end{array}$ & $\begin{array}{l}14.6 \\
14.8 \\
15.9\end{array}$ & $\begin{array}{l}3.9 \\
3.6 \\
3.7\end{array}$ & $\begin{array}{l}1.6 \\
1.7 \\
1.2\end{array}$ \\
\hline
\end{tabular}

TABLE 46. Average Composition of Canister 204 Samples

\begin{tabular}{|c|c|c|c|c|c|c|c|c|c|c|}
\hline \multicolumn{2}{|c|}{ Core Height } & \multicolumn{9}{|c|}{ Oxide wt\% } \\
\hline$m$ & $\mathrm{ft}$ & $\underline{\mathrm{SiO}}_{2}$ & $\mathrm{Al}_{2}-3$ & $\underline{B}_{2} \underline{0}_{3}$ & $\underline{\mathrm{CaO}}$ & $\mathrm{Fe}_{2} \mathrm{O}_{3}$ & $\mathrm{Li}_{2} \mathrm{O}-$ & $\underline{\mathrm{Na}}_{2} \underline{0}$ & $\underline{\mathrm{MnO}}_{2}$ & $\underline{\mathrm{NiO}}$ \\
\hline Defined & & 44.0 & 3.7 & 8.0 & 5.1 & 14.7 & 3.2 & 15.5 & 3.9 & 1.7 \\
\hline 0 & 0 & 45.7 & 3.6 & 8.0 & 5.0 & 13.1 & 3.3 & 15.3 & 3.7 & 1.6 \\
\hline 0.30 & 1 & 45.9 & 3.6 & 8.0 & 5.1 & 13.4 & 3.1 & 14.8 & 3.9 & 1.5 \\
\hline 0.61 & 2 & 45.6 & 3.7 & 8.0 & 5.0 & 13.5 & 3.2 & 15.0 & 4.0 & 1.4 \\
\hline 0.91 & 3 & 45.4 & 3.7 & 8.0 & 5.0 & 13.6 & 3.2 & 15.2 & 4.0 & 1.4 \\
\hline 1.21 & 4 & 44.9 & 3.8 & 8.0 & 5.1 & 14.1 & 3.1 & 14.7 & 4.1 & 1.6 \\
\hline 1.52 & 5 & 45.6 & 3.8 & 8.0 & 5.0 & 13.4 & 3.2 & 15.1 & 3.7 & 1.5 \\
\hline Core & pth & & & & & & & & & \\
\hline $\mathrm{cm}$ & in. & & & & & & & & & \\
\hline $0-11.4$ & $0-4.5$ & 45.6 & 3.7 & 8.0 & 5.0 & 13.6 & 3.1 & 14.8 & 4.0 & 1.6 \\
\hline $11.4-22.9$ & $4.5-9.0$ & 45.4 & 3.7 & 8.0 & 5.0 & 13.4 & 3.2 & 15.3 & 3.8 & 1.6 \\
\hline $22.9-34.3$ & $9.0-13.5$ & 45.6 & 3.8 & 8.0 & 5.0 & 13.4 & 3.2 & 15.2 & 3.9 & 1.3 \\
\hline $34.3-45.7$ & $13.5-18.0$ & 45.4 & 3.8 & 8.0 & 5.1 & 13.9 & 3.1 & 14.6 & 4.0 & 1.4 \\
\hline $\begin{array}{l}\text { AVE. } \\
\text { STD. DEV. }\end{array}$ & & $\begin{array}{r}45.5 \\
0.4\end{array}$ & $\begin{array}{l}3.7 \\
0.1\end{array}$ & $\begin{array}{l}8.0 \\
0.1\end{array}$ & $\begin{array}{l}5.0 \\
0.1\end{array}$ & $\begin{array}{r}13.5 \\
0.5\end{array}$ & $\begin{array}{l}3.2 \\
0.1\end{array}$ & $\begin{array}{r}15.0 \\
0.5\end{array}$ & $\begin{array}{l}3.9 \\
0.2\end{array}$ & $\begin{array}{l}1.5 \\
0.2\end{array}$ \\
\hline
\end{tabular}


TABLE 47. Crystalline Content of Canister 204 Samples

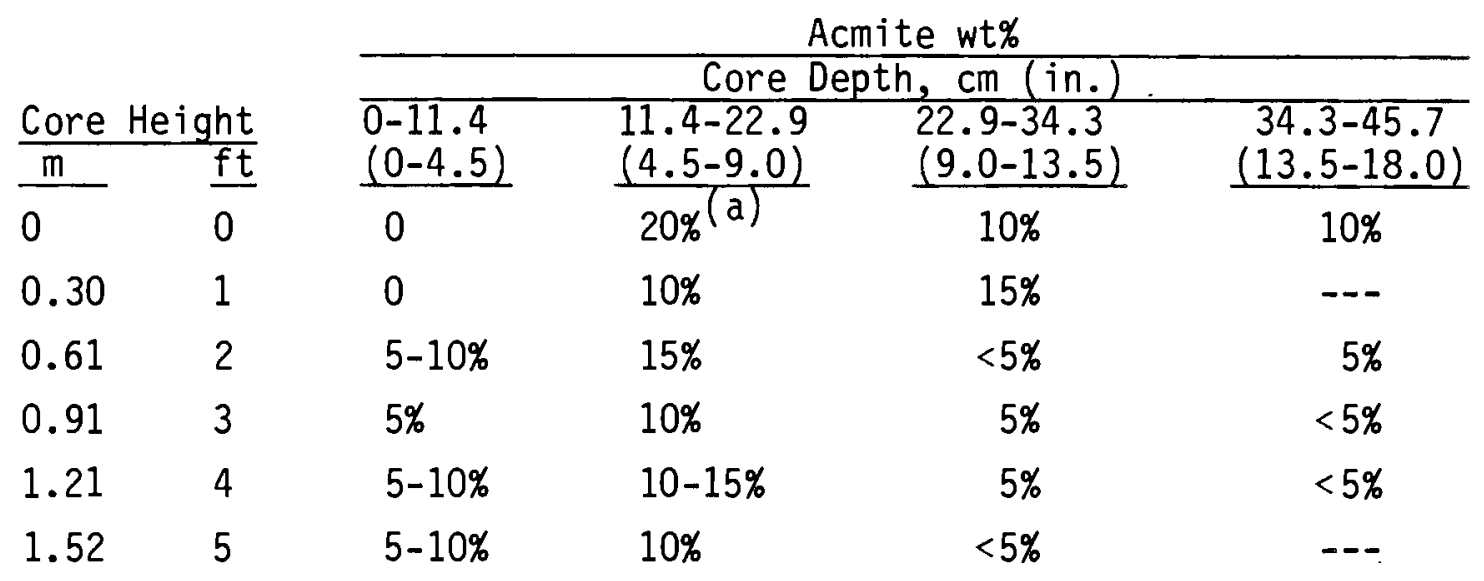

(a) Acmite $\mathrm{NaFeSi}_{2} \mathrm{O}_{6}$

CFCM-8 (NOVEMBER 1979)

Melting trial CFCM-8 converted 19,180 L of simulated liquid waste into $4220 \mathrm{~kg}$ of simulated defense waste glass. The objective of this trial was to determine the impact of reducing agents and improved waste/frit mixing on foaming in the CFCM. Cornstarch at 1 to $1-1 / 2$ wt\% on a glass basis was added as the reducing agent first in the liquid feed and later mixed with the frit. The location of the frit addition line was moved nearer the filters in the spray calciner for better mixing of the calcine and frit. Some of the $\mathrm{Na}_{2} \mathrm{O}$ and $\mathrm{SiO}_{2}$ from the frit was initially incorporated in the liquid feed to improve mixing. As a result, the frit composition was altered to produce the standard TDS-211 waste glass composition. These modifications did not significantly reduce foaming or improve process melting rates. The average melting rate was approximately $53 \mathrm{~kg} / \mathrm{h}\left(70 \mathrm{~kg} / \mathrm{h} / \mathrm{m}^{2}\right)$.

A liquid-feed and waste-glass sampling program similar to that used in CFCM- 6 and CFCM-7 was used. Table 50 lists the composition of liquid feed samples collected during the trial. The two defined waste compositions, Parts I and II, reflect the initial addition of sodium silicate $\left(\mathrm{Na}_{2} \mathrm{O}\right.$ and $\left.\mathrm{SiO}_{2}\right)$ to the liquid feed and its subsequent removal. Considerable variation was noted for many of the feed constituents. The large variation in $\mathrm{MnO}_{2}$ and a significant 

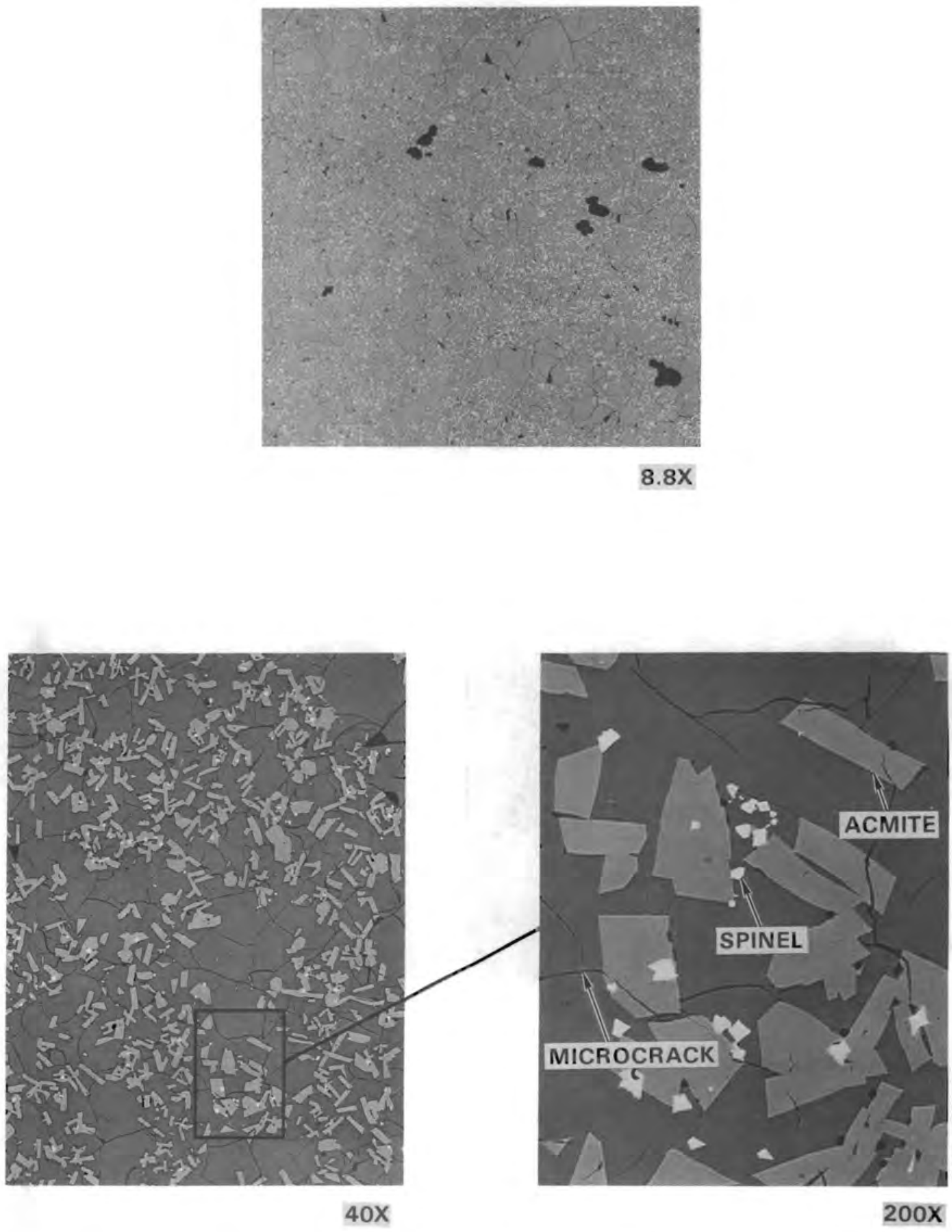

FIGURE 18. Microstructure of Canister 204 Sample [Height $0.61 \mathrm{~m}(2 \mathrm{ft})$; Depth 11.4-22.9 cm (4.5-9.0 in.)] 


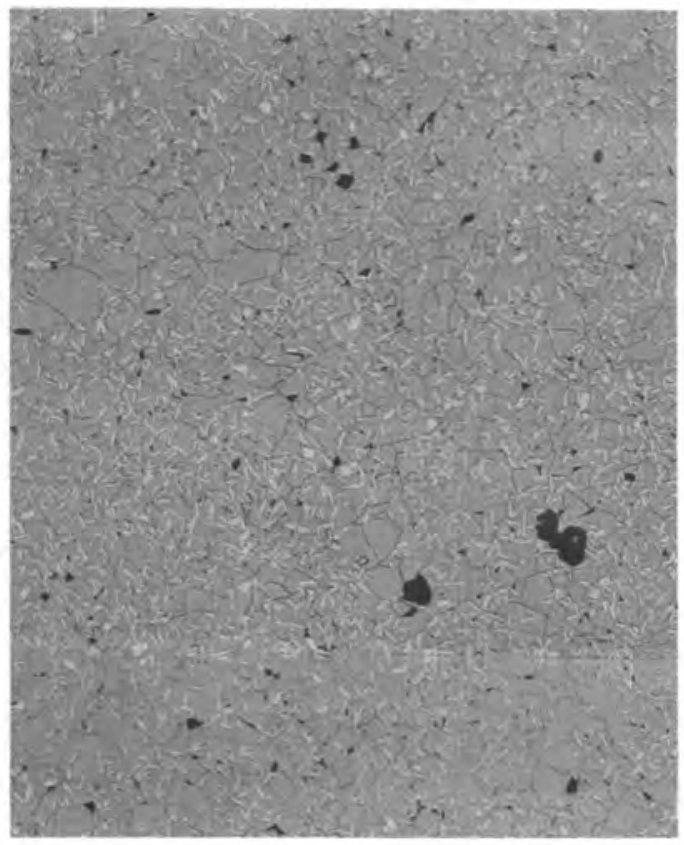

$8.8 \mathrm{X}$

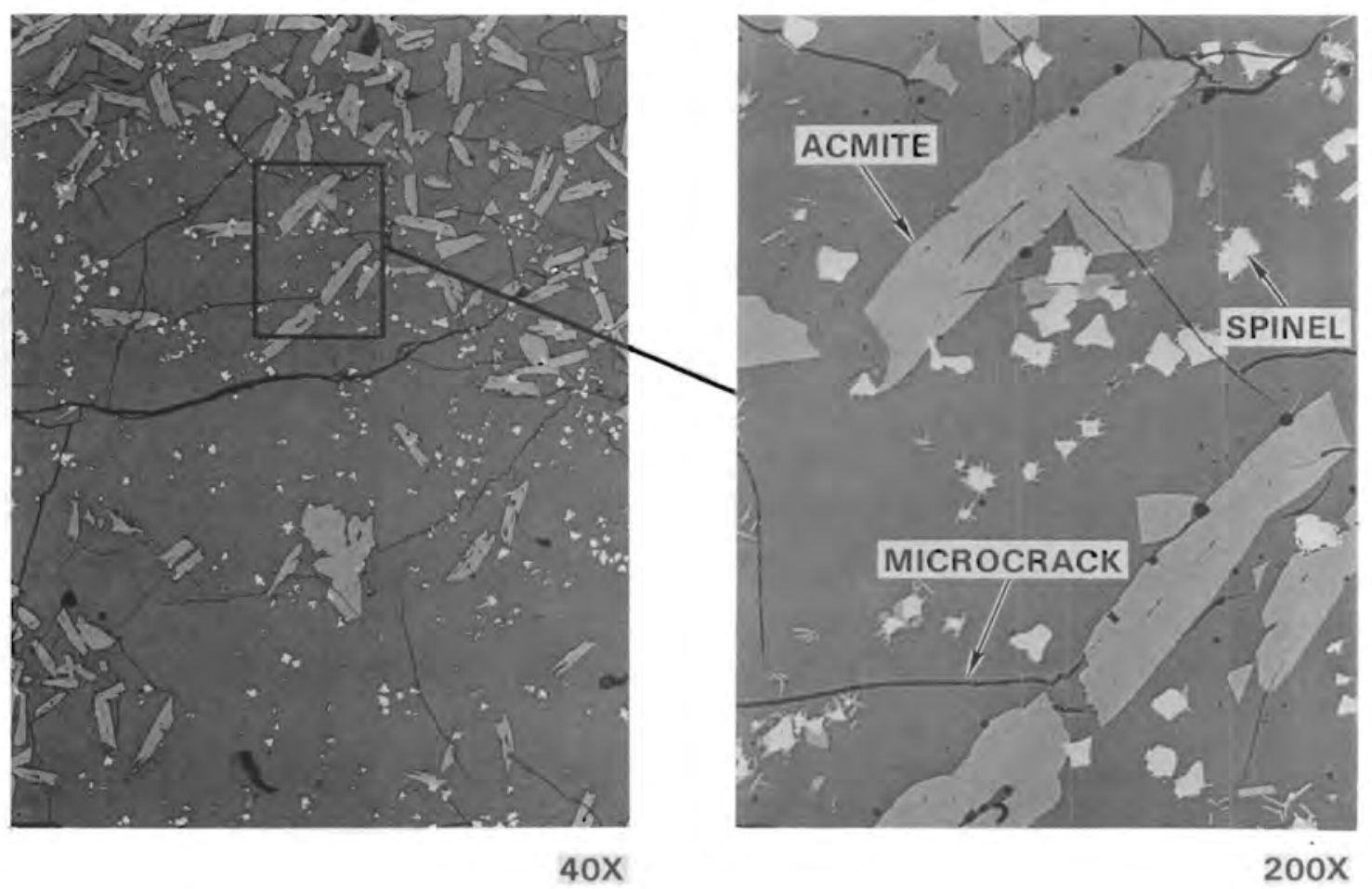

FIGURE 19. Microstructure of Canister 204 Sample [Height $1.21 \mathrm{~m}(4 \mathrm{ft})$; Depth 11.4-22.9 cm (4.5-9.0 in.)] 
TABLE 48. Weight Loss of Canister 204 Samples Under pH4 Conditions wt\% Loss

\begin{tabular}{|c|c|c|c|c|c|c|}
\hline \multirow{2}{*}{\multicolumn{2}{|c|}{$\frac{\text { Core Height }}{\mathrm{m}}$}} & \multicolumn{4}{|c|}{ wt\% Loss } & \multirow[b]{2}{*}{ Ave. } \\
\hline & & $\begin{array}{l}0-11.4 \\
(0-4.5) \\
\end{array}$ & $\begin{array}{r}\text { Core } \\
11.4-22 . \\
(4.5-9.0) \\
\end{array}$ & $\begin{array}{l}h, \mathrm{~cm} \text { (in.) } \\
22.9-34.3 \\
(9.0-13.5)\end{array}$ & $\begin{array}{r}34.3-45.7 \\
(13.5-18.0)\end{array}$ & \\
\hline 0 & 0 & 81.2 & 58.5 & 66.2 & 69.6 & 68.9 \\
\hline 0.30 & 1 & 81.5 & 73.5 & 71.4 & -- & 75.5 \\
\hline 0.61 & 2 & 60.1 & 60.6 & 76.6 & 73.2 & 67.6 \\
\hline 0.91 & 3 & 85.1 & 62.2 & 76.2 & 85.0 & 77.2 \\
\hline 1.21 & 4 & 71.9 & 66.3 & 86.6 & 92.1 & 79.5 \\
\hline 1.52 & 5 & 81.3 & 83.4 & 83.3 & --- & 82.3 \\
\hline AVE. & & 76.8 & 67.4 & 76.7 & 80.3 & \\
\hline
\end{tabular}

Mean pH4 Weight Loss $74.8 \pm 9.8$ wt\%

TABLE 49. Weight Loss of Canister 204 Samples Under Soxhlet Conditions

\begin{tabular}{|c|c|c|c|c|c|c|}
\hline \multirow[b]{2}{*}{$\frac{\text { Core }}{m}$} & \multirow[b]{2}{*}{$\frac{\text { Height }}{\mathrm{ft}}$} & \multicolumn{4}{|c|}{ wt\% Loss } & \multirow[b]{2}{*}{ Ave. } \\
\hline & & $\begin{array}{l}0-11.4 \\
(0-4.5)\end{array}$ & $\begin{array}{c}\text { Core } \\
11.4-22.9 \\
(4.5-9.0)\end{array}$ & $\begin{array}{l}7, \mathrm{~cm}(\mathrm{in} .) \\
22.9-34.3 \\
(9.0-13.5)\end{array}$ & $\begin{array}{c}34.3-45.7 \\
(13.5-18.0)\end{array}$ & \\
\hline 0 & 0 & 1.88 & 6.36 & 4.66 & 4.49 & 4.35 \\
\hline 0.30 & 1 & 2.02 & 3.57 & 3.73 & --- & 3.11 \\
\hline 0.61 & 2 & 4.13 & 6.39 & 2.26 & 6.84 & 4.91 \\
\hline 0.91 & 3 & 2.03 & 5.18 & 2.15 & 2.24 & 2.90 \\
\hline 1.21 & 4 & 3.88 & 4.56 & 2.12 & 2.21 & 3.19 \\
\hline 1.52 & 5 & 2.60 & 2.42 & 2.64 & --- & 2.55 \\
\hline AVE. & & 2.76 & 4.75 & 2.93 & 3.95 & \\
\hline
\end{tabular}

Mean Soxhlet Weight Loss $3.56 \pm 1.58$ wt\%

portion of the total oxide variation is due to sampling error with the dense $\mathrm{MnO}_{2}$ particles. As in previous melting trials, the liquid feed specific gravity showed little relationship to feed composition or oxide content. The feed $\mathrm{pH}$ dropped rapidly with the removal of sodium silicate as shown in Figure 20. Little mixing of the two liquid feed compositions occurred in the process. 
TABLE 50. Oxide Composition of CFCM-8 Liquid Feed Samples

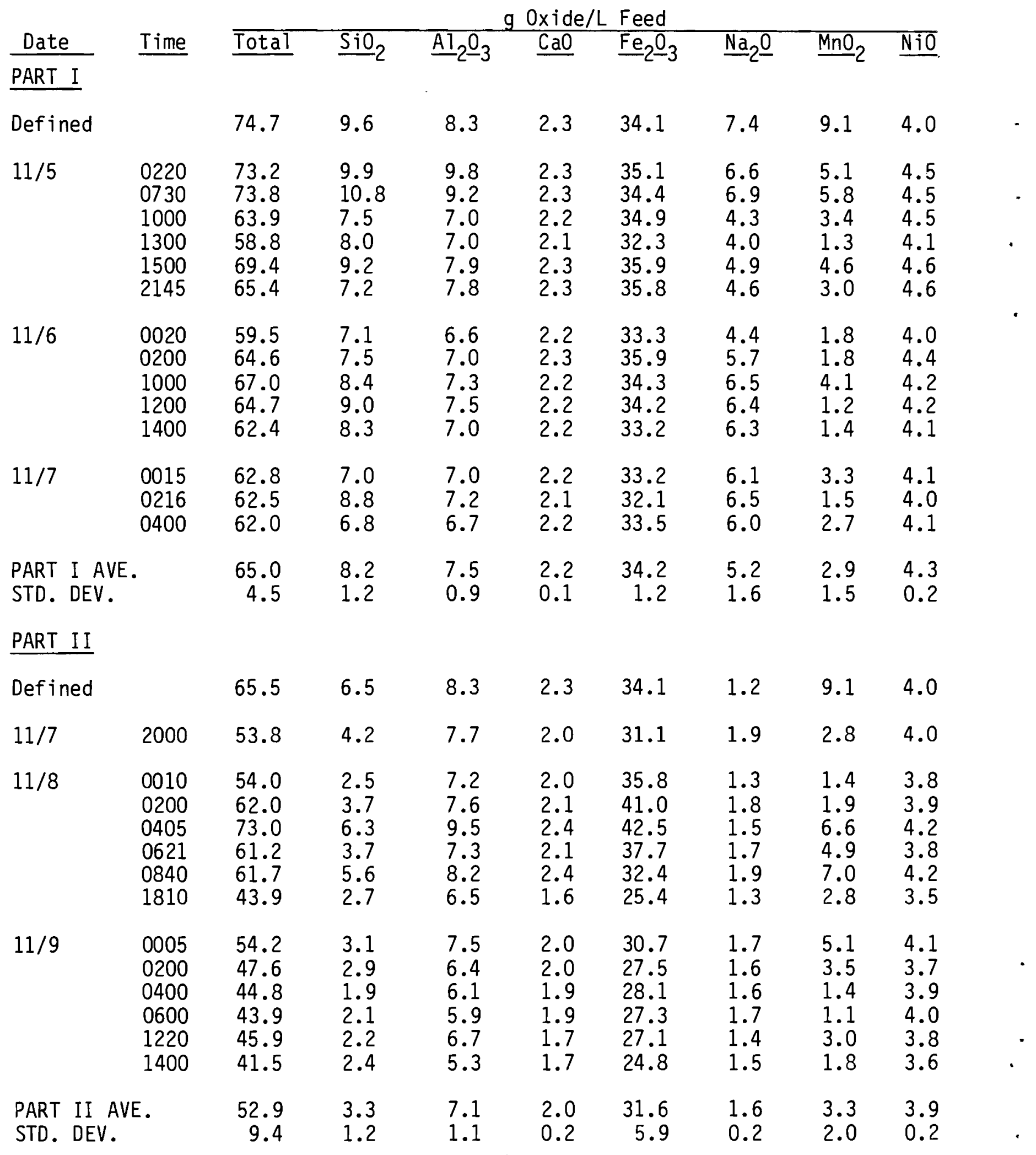




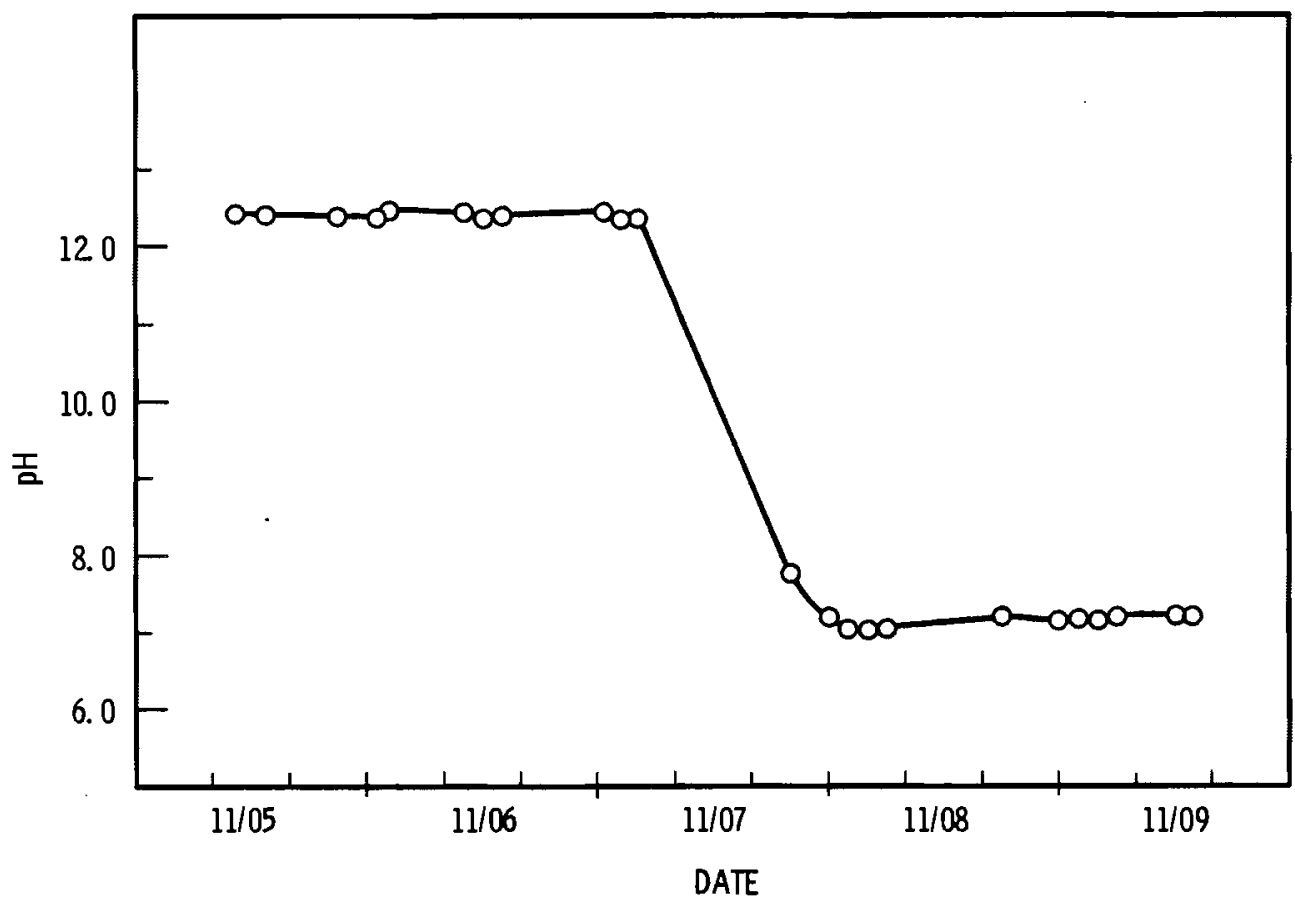

FIGURE 20. $\mathrm{pH}$ of CFCM-8 Liquid Feed Samples

The waste glass produced during the CFCM- 8 melting trial was slightly deficient in waste components containing an average estimated 23 wt\% waste oxides compared to the target $28 \mathrm{wt} \%$. The composition of the wastes component in the glass showed significantly reduced levels of $\mathrm{Fe}_{2} \mathrm{O}_{3}$ and $\mathrm{NiO}$ with increased levels of $\mathrm{MnO}_{2}$ ( $\mathrm{Table} \mathrm{51)}$ ). This is not consistent with the liquid feed analysis which indicated near target levels of $\mathrm{Fe}_{2} \mathrm{O}_{3}$ and $\mathrm{NiO}$. The formation and separation of a Fe-Ni spinel crystalline phase in the glass would decrease to some degree the quantities of $\mathrm{Fe}_{2} \mathrm{O}_{3}$ and $\mathrm{NiO}$ in the waste glass. In only one instance, however, were spinel crystals identified in a grab sample. All others contained no crystalline material. With no estimate of the quantity of spinel accumulated in the melter during the trial, this possibility remains unconfirmed.

The last canister filled, 113, was core-drilled for sample analysis. The normalized composition of these samples is 1isted in Table 52. The averages in Table 53 show a significant decrease in all waste components except $\mathrm{MnO}_{2}$ 
TABLE 51. Normalized Composition of CFCM-8 Grab Samples

\begin{tabular}{|c|c|c|c|c|c|c|c|c|c|c|}
\hline \multirow[b]{2}{*}{ Date } & \multirow[b]{2}{*}{ Time } & \multicolumn{9}{|c|}{ 0xide wt\% } \\
\hline & & $\overline{\mathrm{SiO}_{2}}$ & $\mathrm{Al}_{2} \underline{\mathrm{O}}_{3}$ & $\underline{B}_{2} \underline{O}_{3}$ & $\mathrm{CaO}$ & $\mathrm{Fe}_{2} \underline{O}_{3}$ & $\underline{L i}_{2} \underline{0}$ & $\underline{\mathrm{Na}} \underline{a}_{2} \underline{0}$ & $\underline{\mathrm{MnO}} 2$ & $\underline{\mathrm{Ni} 0}$ \\
\hline Defined & & 44.0 & 3.7 & 8.0 & 5.1 & 14.7 & 3.2 & 15.5 & $3.9^{6}$ & 1.7 \\
\hline $11 / 5 / 79$ & $\begin{array}{l}0330 \\
0830 \\
1350 \\
2330\end{array}$ & $\begin{array}{l}45.6 \\
46.5 \\
47.6 \\
48.1\end{array}$ & $\begin{array}{l}4.5 \\
4.3 \\
4.2 \\
3.7\end{array}$ & $\begin{array}{l}7.9 \\
8.0 \\
8.3 \\
8.5\end{array}$ & $\begin{array}{l}5.3 \\
5.2 \\
5.0 \\
5.0\end{array}$ & $\begin{array}{l}14.0 \\
12.7 \\
11.5 \\
10.8\end{array}$ & $\begin{array}{l}2.9 \\
3.0 \\
3.1 \\
3.2\end{array}$ & $\begin{array}{l}14.8 \\
14.6 \\
14.3 \\
14.6\end{array}$ & $\begin{array}{l}3.5 \\
4.2 \\
4.5 \\
4.6\end{array}$ & $\begin{array}{l}1.2 \\
1.2 \\
1.2 \\
1.3\end{array}$ \\
\hline $11 / 6 / 79$ & $\begin{array}{l}0330 \\
0900 \\
1400 \\
1800\end{array}$ & $\begin{array}{l}48.2 \\
48.2 \\
48.8\end{array}$ & $\begin{array}{l}3.5 \\
4.9 \\
3.3\end{array}$ & $\begin{array}{l}8.7 \\
8.6 \\
8.9\end{array}$ & $\begin{array}{l}5.0 \\
4.9 \\
4.8 \\
\text { CANI }\end{array}$ & $\begin{array}{l}10.7 \\
9.8 \\
10.3 \\
\text { CER } 209\end{array}$ & $\begin{array}{l}3.1 \\
3.2 \\
3.5 \\
\text { OUT }\end{array}$ & $\begin{array}{l}14.6 \\
15.0 \\
14.7\end{array}$ & $\begin{array}{l}4.6 \\
4.1 \\
4.2\end{array}$ & $\begin{array}{l}1.3 \\
1.2 \\
1.3\end{array}$ \\
\hline $11 / 7 / 79$ & $\begin{array}{l}0122 \\
0537 \\
1725 \\
2050 \\
2340\end{array}$ & $\begin{array}{l}46.9 \\
47.4 \\
47.4 \\
47.8 \\
47.8\end{array}$ & $\begin{array}{l}3.3 \\
3.4 \\
3.4 \\
3.4 \\
3.3\end{array}$ & $\begin{array}{l}8.6 \\
8.7 \\
8.5 \\
8.5 \\
8.6\end{array}$ & $\begin{array}{l}4.8 \\
4.7 \\
4.8 \\
4.6 \\
4.8\end{array}$ & $\begin{array}{l}10.9 \\
10.7 \\
11.3 \\
10.8 \\
10.8\end{array}$ & $\begin{array}{l}3.4 \\
3.5 \\
3.2 \\
3.3 \\
3.0\end{array}$ & $\begin{array}{l}15.9 \\
15.6 \\
15.2 \\
15.3 \\
15.5\end{array}$ & $\begin{array}{l}4.5 \\
4.3 \\
4.4 \\
4.6 \\
4.5\end{array}$ & $\begin{array}{l}1.4 \\
1.4 \\
1.5 \\
1.4 \\
1.4\end{array}$ \\
\hline $11 / 8 / 79$ & $\begin{array}{l}0530 \\
0945 \\
1100\end{array}$ & $\begin{array}{l}48.8 \\
46.5\end{array}$ & $\begin{array}{l}3.1 \\
3.4\end{array}$ & $\begin{array}{l}8.7 \\
8.6\end{array}$ & $\begin{array}{l}4.5 \\
4.7 \\
\text { CANI }\end{array}$ & $\begin{array}{l}10.2 \\
13.4 \\
\text { TER } 115\end{array}$ & $\begin{array}{r}3.9 \\
3.1 \\
\text { OUT }\end{array}$ & $\begin{array}{l}16.4 \\
14.7\end{array}$ & $\begin{array}{l}2.9 \\
3.8\end{array}$ & $\begin{array}{l}1.3 \\
1.5\end{array}$ \\
\hline & $\begin{array}{l}1130 \\
1500\end{array}$ & 46.4 & 3.4 & 8.4 & $\begin{array}{l}4.7 \\
\text { CANI }\end{array}$ & $\begin{array}{l}12.5 \\
\text { TER } 114\end{array}$ & ${ }_{\text {OUT }}^{3.2}$ & 15.5 & 4.2 & 1.4 \\
\hline & 2230 & 46.9 & 3.5 & 8.6 & 4.7 & 11.8 & 3.2 & 15.3 & 4.3 & 1.5 \\
\hline $11 / 9 / 79$ & $\begin{array}{l}0116 \\
0444 \\
0650 \\
1200 \\
1600\end{array}$ & $\begin{array}{l}47.5 \\
48.7 \\
48.6 \\
48.5\end{array}$ & $\begin{array}{l}3.2 \\
3.0 \\
3.0 \\
3.2\end{array}$ & $\begin{array}{l}8.8 \\
9.1 \\
8.9 \\
8.8\end{array}$ & $\begin{array}{l}4.8 \\
4.8 \\
4.9 \\
4.8 \\
\text { CANI }\end{array}$ & $\begin{array}{l}10.7 \\
9.0 \\
9.1 \\
9.1 \\
\text { TER } 113\end{array}$ & $\begin{array}{r}3.4 \\
3.4 \\
3.4 \\
3.5 \\
\text { OUT }\end{array}$ & $\begin{array}{l}15.9 \\
16.0 \\
16.5 \\
16.6\end{array}$ & $\begin{array}{l}4.2 \\
4.6 \\
4.4 \\
4.1\end{array}$ & $\begin{array}{l}1.4 \\
1.2 \\
1.2 \\
1.2\end{array}$ \\
\hline $\begin{array}{l}\text { AVE. } \\
\text { STD. DEV. }\end{array}$ & & $\begin{array}{r}47.6 \\
0.9\end{array}$ & $\begin{array}{l}3.6 \\
0.5\end{array}$ & $\begin{array}{l}8.6 \\
0.3\end{array}$ & $\begin{array}{l}4.8 \\
0.2\end{array}$ & $\begin{array}{r}11.0 \\
1.4\end{array}$ & $\begin{array}{l}3.3 \\
0.2\end{array}$ & $\begin{array}{r}15.4 \\
0.7\end{array}$ & $\begin{array}{l}4.2 \\
0.4\end{array}$ & $\begin{array}{l}1.3 \\
0.1\end{array}$ \\
\hline $\begin{array}{l}\text { CANISTER } \\
\text { AVE. } \\
\text { STD. DEV. }\end{array}$ & 13 & $\begin{array}{r}48.0 \\
0.8\end{array}$ & $\begin{array}{l}3.2 \\
0.2\end{array}$ & $\begin{array}{l}8.8 \\
0.2\end{array}$ & $\begin{array}{l}4.8 \\
0.1\end{array}$ & $\begin{array}{l}9.9 \\
1.2\end{array}$ & $\begin{array}{l}3.4 \\
0.1\end{array}$ & $\begin{array}{r}16.1 \\
0.5\end{array}$ & $\begin{array}{l}4.2 \\
0.2\end{array}$ & $\begin{array}{l}1.3 \\
0.2\end{array}$ \\
\hline
\end{tabular}

above the $0.61-\mathrm{m}(2-\mathrm{ft})$ level. The analysis of grab samples collected during filling of canister 113 show the same decrease in waste content. Micrographs of the low waste content product above the $0.61-m(2-f t)$ level show an 
TABLE 52. Normalized Composition of Canister 113 Samples

\begin{tabular}{|c|c|c|c|c|c|c|c|c|c|c|c|c|}
\hline \multicolumn{2}{|c|}{$\begin{array}{c}\text { Core } \\
\text { Height }\end{array}$} & \multicolumn{2}{|c|}{ Core Depth } & \multicolumn{9}{|c|}{ Oxide wt\% } \\
\hline$m$ & $\overline{f t}$ & $\mathrm{~cm}$ & in. & $\overline{\mathrm{SiO}} 2$ & $\mathrm{Al}_{2}{ }_{-3}$ & $\underline{B}_{2} \underline{O}_{3}$ & $\mathrm{CaO}$ & $\underline{F e}_{2}-_{3}$ & $\underline{\mathrm{Li}}_{2} \underline{\mathrm{O}}$ & $\underline{\mathrm{Na}}_{2} \underline{\mathrm{O}}$ & $\underline{\mathrm{MnO}} 2$ & NiO \\
\hline \multicolumn{2}{|c|}{ Defined } & & & 44.0 & 3.7 & 8.0 & 5.0 & 14.5 & 3.2 & 15.5 & 3.9 & 1.7 \\
\hline 0 & 0 & $\begin{array}{l}0-7.6 \\
7.6-15.2 \\
15.2-22.9 \\
22.9-30.5\end{array}$ & $\begin{array}{l}0-3 \\
3-6 \\
6-9 \\
9-12\end{array}$ & $\begin{array}{l}45.4 \\
45.7 \\
46.4 \\
46.5\end{array}$ & $\begin{array}{l}3.5 \\
3.4 \\
3.4 \\
3.3\end{array}$ & $\begin{array}{l}8.0 \\
8.0 \\
8.3 \\
8.3\end{array}$ & $\begin{array}{l}4.8 \\
4.7 \\
4.8 \\
4.9\end{array}$ & $\begin{array}{l}13.3 \\
12.4 \\
12.2 \\
12.4\end{array}$ & $\begin{array}{l}3.3 \\
3.4 \\
3.3 \\
3.2\end{array}$ & $\begin{array}{l}15.5 \\
16.0 \\
15.7 \\
15.4\end{array}$ & $\begin{array}{l}4.3 \\
4.2 \\
4.1 \\
4.2\end{array}$ & $\begin{array}{l}1.7 \\
1.6 \\
1.6 \\
1.6\end{array}$ \\
\hline 0.30 & 1 & $\begin{array}{l}0-7.6 \\
7.6-15.2 \\
15.2-22.9 \\
22.9-30.5\end{array}$ & $\begin{array}{l}0-3 \\
3-6 \\
6-9 \\
9-12\end{array}$ & $\begin{array}{l}46.6 \\
47.1 \\
46.8 \\
46.7\end{array}$ & $\begin{array}{l}3.3 \\
3.3 \\
3.3 \\
3.4\end{array}$ & $\begin{array}{l}8.4 \\
8.5 \\
8.4 \\
8.2\end{array}$ & $\begin{array}{l}4.8 \\
4.9 \\
4.9 \\
4.9\end{array}$ & $\begin{array}{l}11.8 \\
11.5 \\
11.8 \\
12.1\end{array}$ & $\begin{array}{l}3.3 \\
3.3 \\
3.3 \\
3.1\end{array}$ & $\begin{array}{l}15.8 \\
15.6 \\
15.8 \\
15.5\end{array}$ & $\begin{array}{l}4.2 \\
4.2 \\
4.2 \\
4.2\end{array}$ & $\begin{array}{l}1.5 \\
1.5 \\
1.4 \\
1.6\end{array}$ \\
\hline 0.61 & 2 & $\begin{array}{l}0-7.6 \\
7.6-15.2 \\
15.2-22.9 \\
22.9-30.5\end{array}$ & $\begin{array}{l}0-3 \\
3-6 \\
6-9 \\
9-12\end{array}$ & $\begin{array}{l}48.5 \\
48.9 \\
48.9 \\
48.9\end{array}$ & $\begin{array}{l}3.0 \\
3.0 \\
2.9 \\
2.9\end{array}$ & $\begin{array}{l}8.7 \\
8.8 \\
8.7 \\
8.8\end{array}$ & $\begin{array}{l}5.0 \\
5.0 \\
5.0 \\
5.0\end{array}$ & $\begin{array}{l}9.9 \\
9.7 \\
9.2 \\
9.2\end{array}$ & $\begin{array}{l}3.3 \\
3.2 \\
3.4 \\
3.4\end{array}$ & $\begin{array}{l}15.8 \\
15.5 \\
16.2 \\
16.0\end{array}$ & $\begin{array}{l}4.3 \\
4.4 \\
4.3 \\
4.4\end{array}$ & $\begin{array}{l}1.3 \\
1.3 \\
1.2 \\
1.2\end{array}$ \\
\hline 0.91 & 3 & $\begin{array}{l}0-7.6 \\
7.6-15.2 \\
15.2-22.9 \\
22.9-30.5\end{array}$ & $\begin{array}{l}0-3 \\
3-6 \\
6-9 \\
9-12\end{array}$ & $\begin{array}{l}49.3 \\
49.4 \\
49.4 \\
48.9\end{array}$ & $\begin{array}{l}2.9 \\
3.0 \\
3.1 \\
3.0\end{array}$ & $\begin{array}{l}8.8 \\
8.9 \\
8.8 \\
8.7\end{array}$ & $\begin{array}{l}5.0 \\
5.0 \\
5.1 \\
4.8\end{array}$ & $\begin{array}{l}9.0 \\
9.1 \\
9.2 \\
8.9\end{array}$ & $\begin{array}{l}3.4 \\
3.5 \\
3.4 \\
3.6\end{array}$ & $\begin{array}{l}15.8 \\
15.3 \\
15.2 \\
16.5\end{array}$ & $\begin{array}{l}4.3 \\
4.3 \\
4.3 \\
4.1\end{array}$ & $\begin{array}{l}1.2 \\
1.2 \\
1.2 \\
1.2\end{array}$ \\
\hline 1.21 & 4 & $\begin{array}{l}0-7.6 \\
7.6-15.2 \\
15.2-22.9 \\
22.9-30.5\end{array}$ & $\begin{array}{l}0-3 \\
3-6 \\
6-9 \\
9-12\end{array}$ & $\begin{array}{l}48.5 \\
49.2 \\
48.8 \\
48.8\end{array}$ & $\begin{array}{l}3.0 \\
3.0 \\
3.0 \\
3.0\end{array}$ & $\begin{array}{l}8.7 \\
8.8 \\
8.8 \\
8.7\end{array}$ & $\begin{array}{l}4.8 \\
5.0 \\
5.0 \\
4.9\end{array}$ & $\begin{array}{l}8.8 \\
9.1 \\
9.1 \\
9.0\end{array}$ & $\begin{array}{l}3.6 \\
3.5 \\
3.4 \\
3.5\end{array}$ & $\begin{array}{l}17.3 \\
15.9 \\
16.2 \\
16.5\end{array}$ & $\begin{array}{l}3.9 \\
4.0 \\
4.1 \\
4.0\end{array}$ & $\begin{array}{l}1.2 \\
1.3 \\
1.2 \\
1.2\end{array}$ \\
\hline 1.52 & 5 & $\begin{array}{l}0-7.6 \\
7.6-15.2 \\
15.2-22.9 \\
22.9-30.5\end{array}$ & $\begin{array}{l}0-3 \\
3-6 \\
6-9 \\
9-12\end{array}$ & $\begin{array}{l}48.4 \\
48.9 \\
48.7 \\
48.9\end{array}$ & $\begin{array}{l}3.1 \\
3.0 \\
3.0 \\
3.0\end{array}$ & $\begin{array}{l}8.7 \\
8.8 \\
8.8 \\
8.8\end{array}$ & $\begin{array}{l}4.9 \\
4.9 \\
4.9 \\
5.0\end{array}$ & $\begin{array}{l}9.3 \\
8.8 \\
9.0 \\
9.2\end{array}$ & $\begin{array}{l}3.4 \\
3.6 \\
3.6 \\
3.5\end{array}$ & $\begin{array}{l}16.4 \\
16.4 \\
16.2 \\
16.0\end{array}$ & $\begin{array}{l}4.2 \\
4.0 \\
4.3 \\
4.1\end{array}$ & $\begin{array}{l}1.3 \\
1.2 \\
1.3 \\
1.3\end{array}$ \\
\hline
\end{tabular}

inhomogeneous distribution of fine acmite crystals in a glass matrix (Figures 21 and 22). While some porosity is present, there is an absence of microcracking. There are also very few spinel crystals in these samples. X-ray data in Table 54 shows that the major compositional change in the canister did not significantly alter the crystalline phase present. While samples near the wall were generally amorphous, more central samples contained from 5.8 to 16.5 wt\% acmite crystals.

Compositional changes, however, directly affected the glass durability under $\mathrm{pH} 4$ conditions. As waste content decreased, $\mathrm{pH} 4$ durability increased 
TABLE 53. Average Composition of Canister 113 Samples

\begin{tabular}{|c|c|c|c|c|c|c|c|c|c|c|}
\hline \multicolumn{2}{|c|}{ Core Height } & \multicolumn{9}{|c|}{ Oxide wt\% } \\
\hline $\mathrm{m}$ & $\mathrm{ft}$ & $\overline{\mathrm{SiO}}$ & $\mathrm{Al}_{2} \underline{O}_{3}$ & $\underline{B}_{2} \underline{O}_{3}$ & $\overrightarrow{\mathrm{CaO}}$ & $\mathrm{Fe}_{2}{ }_{-}{ }_{3}$ & $\underline{\underline{L i}} \underline{\underline{0}}$ & $\underline{\mathrm{Na}} 2$ & MnO & $\overline{\mathrm{NiO}}$ \\
\hline Defined & & 44.0 & 3.7 & 8.0 & 5.1 & 14.7 & 3.2 & 15.5 & 3.9 & \\
\hline 0 & 0 & 46.0 & 3.4 & 8.2 & 4.8 & 12.6 & 3.3 & 15.6 & 4.2 & \\
\hline 0.30 & 1 & 46.8 & 3.3 & 8.4 & 4.9 & 11.8 & 3.2 & 15.7 & 4.2 & \\
\hline 0.61 & 2 & 48.8 & 3.0 & 8.8 & 5.0 & 9.5 & 3.3 & 15.9 & 4.4 & \\
\hline 0.91 & 3 & 49.2 & 3.0 & 8.8 & 5.0 & 9.0 & 3.5 & 15.7 & 4.2 & \\
\hline 1.21 & 4 & 48.8 & 3.0 & 8.8 & 5.0 & 9.0 & 3.5 & 16.5 & 4.0 & \\
\hline 1.52 & 5 & 48.7 & 3.0 & 8.8 & 4.9 & 9.1 & 3.5 & 16.2 & 4.2 & 3 \\
\hline
\end{tabular}

\begin{tabular}{|c|c|c|c|c|c|c|c|c|c|c|}
\hline \multicolumn{2}{|c|}{ Core Depth } & & & & & & & & & \\
\hline $\mathrm{cm}$ & in. & & & & & & & & & \\
\hline $0-7.6$ & $0-3$ & 47.8 & 3.1 & 8.5 & 4.9 & 10.4 & 3.4 & 16.1 & 4.2 & 1.4 \\
\hline $7.6-15.2$ & $3-6$ & 48.2 & 3.1 & 8.6 & 4.9 & 10.1 & 3.4 & 15.8 & 4.2 & 1.4 \\
\hline $15.2-22.9$ & $6-9$ & 48.2 & 3.1 & 8.6 & 5.0 & 10.1 & 3.4 & 15.9 & 4.2 & 1.3 \\
\hline $22.9-30.5$ & $9-12$ & 48.1 & 3.1 & 8.6 & 4.9 & 10.1 & 3.4 & 16.0 & 4.2 & 1.4 \\
\hline $\begin{array}{l}\text { AVE. } \\
\text { STD. DEV. }\end{array}$ & & $\begin{array}{r}48.1 \\
1.3\end{array}$ & $\begin{array}{l}3.1 \\
0.2\end{array}$ & $\begin{array}{l}8.6 \\
0.3\end{array}$ & $\begin{array}{l}4.9 \\
0.1\end{array}$ & $\begin{array}{r}10.1 \\
1.5\end{array}$ & $\begin{array}{l}3.4 \\
0.1\end{array}$ & $\begin{array}{r}15.9 \\
0.5\end{array}$ & $\begin{array}{l}4.2 \\
0.1\end{array}$ & $\begin{array}{l}1.3 \\
0.2\end{array}$ \\
\hline
\end{tabular}

from $87.2 \pm 5.4$ wt\% to $30.6 \pm 7.3 w t \%$. Soxhlet results did not show this behavior with an average weight loss of $2.37 \pm 0.33 \mathrm{wt} \%$. Tables 55 and 56 present weight loss data for $\mathrm{pH} 4$ and soxhlet conditions. Grab samples were similarly tested for pH4 and soxhlet durability. The pH4 weight loss of corresponding canister core and grab samples in Figure 23 shows similar behavior. The average $\mathrm{pH} 4$ and soxhlet weight loss for grab samples were $33.0 \pm 19.2 \mathrm{wt} \%$, and $2.00 \pm 0.26 \mathrm{wt} \%$, respectively. 

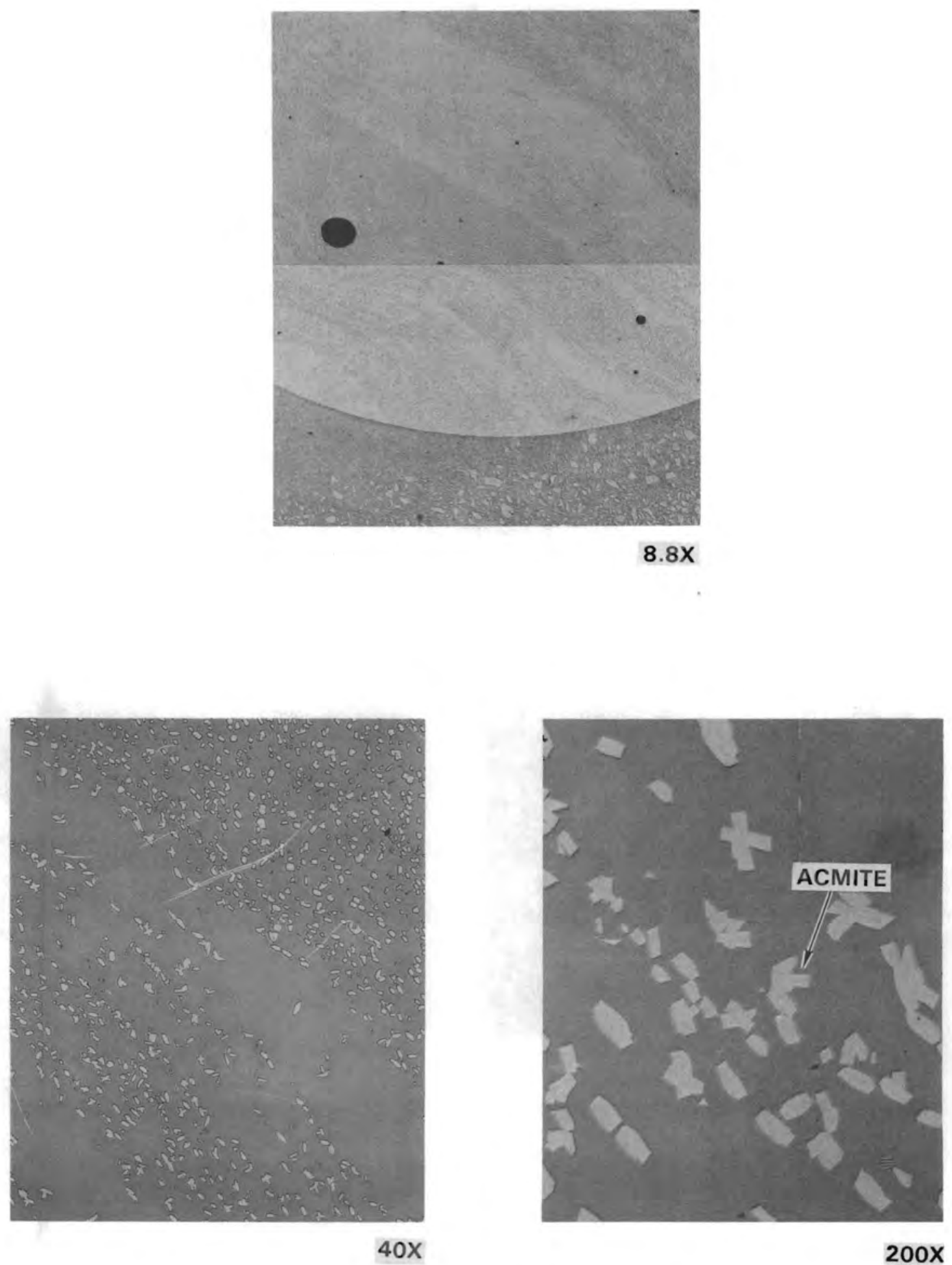

FIGURE 21. Microstructure of Canister 113 Sample [Height $0.61 \mathrm{~m}(2 \mathrm{ft})$; Depth 22.9-30.5 cm (9-12 in.)] 


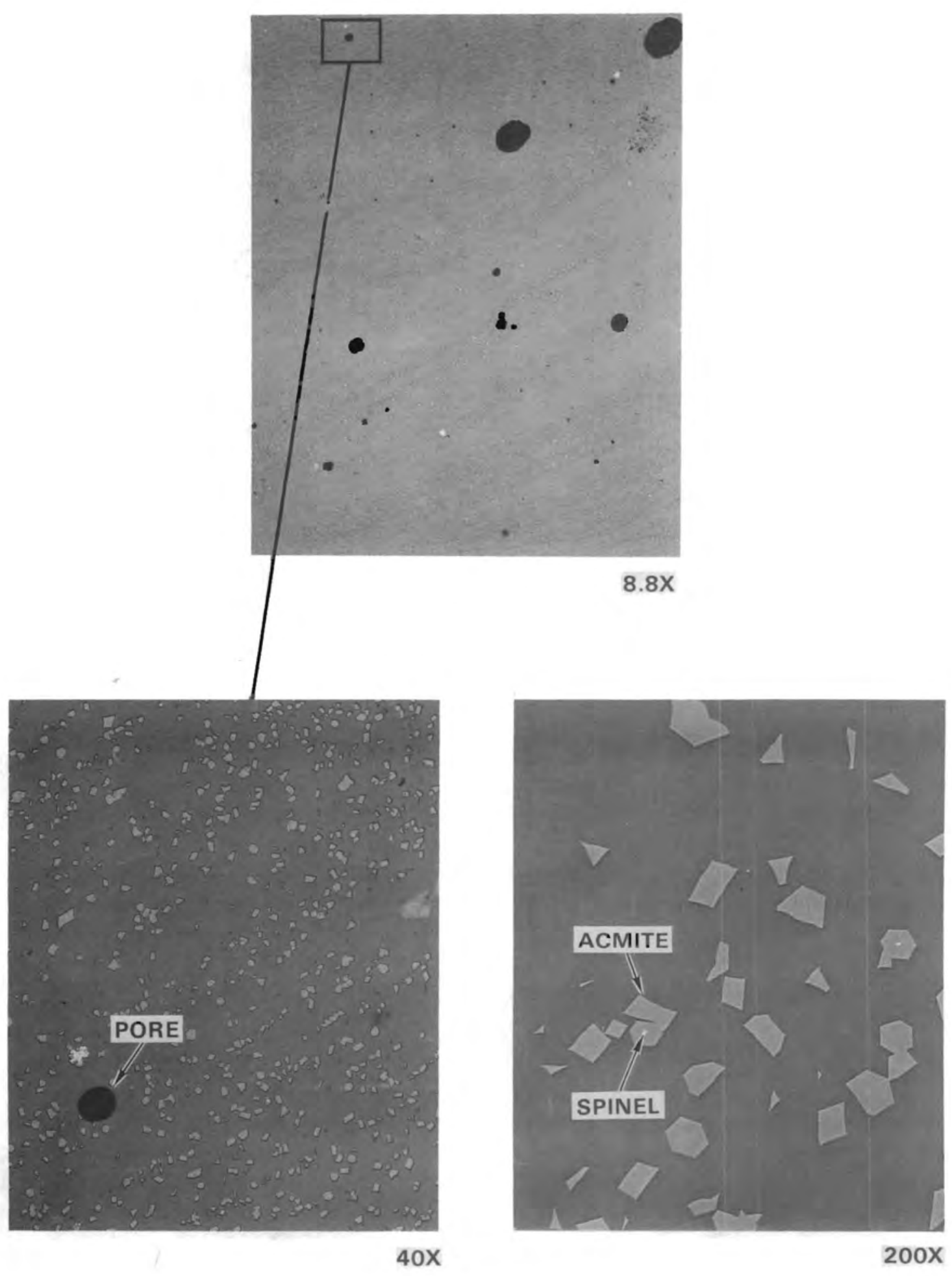

FIGURE 22. Microstructure of Canister 113 Sample [Height $1.21 \mathrm{~m}(4 \mathrm{ft})$; Depth 22.9-30.5 cm (9-12 in.)] 
TABLE 54. Crystalline Content of Canister 113 Samples

\begin{tabular}{|c|c|c|c|c|c|c|}
\hline \multirow[b]{2}{*}{$\frac{\text { Core }}{\mathrm{m}}$} & \multirow[b]{2}{*}{$\frac{\text { Height }}{\mathrm{ft}}$} & \multicolumn{4}{|c|}{ Acmite Crystals ${ }^{(a)}$ wt\% } & \multirow[b]{2}{*}{ Ave. } \\
\hline & & $\begin{array}{l}0-7.6 \\
(0-3) \\
\end{array}$ & $\begin{array}{c}\text { Core } \\
7.6-15.2 \\
(3-6) \\
\end{array}$ & $\begin{array}{c}n, \mathrm{~cm} \text { (in. } \\
15.2-22.9 \\
(6-9)\end{array}$ & $\begin{array}{c}22.9-30.5 \\
(9-12)\end{array}$ & \\
\hline 0 & 0 & 0 & 7.2 & 5.8 & 6.9 & 5.0 \\
\hline 0.30 & 1 & 0 & 0 & 11.5 & 9.8 & 5.3 \\
\hline 0.61 & 2 & 0 & 0 & 7.2 & 14.7 & 7.3 \\
\hline 0.91 & 3 & 0 & 0 & 16.5 & 4.9 & 5.3 \\
\hline 1.21 & 4 & 0 & 10.5 & 7.1 & 8.1 & 6.4 \\
\hline 1.52 & 5 & 0 & 6.6 & 7.1 & 0 & 3.4 \\
\hline AVE. & & 0 & 4.0 & 9.2 & 7.2 & \\
\hline
\end{tabular}

(a) Acmite only crystalline phase identified.

TABLE 55. Weight Loss of Canister 113 Samples Under pH4 Conditions

\begin{tabular}{|c|c|c|c|c|c|c|}
\hline \multirow{3}{*}{\multicolumn{2}{|c|}{ Core Height }} & \multirow{2}{*}{\multicolumn{4}{|c|}{$\frac{\text { wt\% Loss }}{\text { Core Depth cm (in.) }}$}} & \multirow[b]{3}{*}{ Ave. } \\
\hline & & & \multirow{2}{*}{$\frac{\text { Core }}{7.6-15.2}$} & \multirow[b]{2}{*}{$\begin{array}{c}n, \mathrm{~cm}(\mathrm{in} .) \\
15.2-22.9 \\
(6-9)\end{array}$} & \multirow[b]{2}{*}{$\begin{array}{c}22.9-30.5 \\
(9-12)\end{array}$} & \\
\hline & & $\begin{array}{l}0-7.6 \\
(0-3) \\
\end{array}$ & & & & \\
\hline 0 & 0 & 89.9 & 93.0 & 89.7 & 88.2 & 90.2 \\
\hline 0.30 & 1 & 80.7 & 80.4 & 82.2 & 93.5 & 84.2 \\
\hline 0.61 & 2 & 43.1 & 37.8 & 30.0 & 40.6 & 37.9 \\
\hline 0.91 & 3 & 32.1 & 28.2 & 30.5 & 37.9 & 32.2 \\
\hline 1.21 & 4 & 31.9 & 25.8 & 32.6 & 31.2 & 30.4 \\
\hline 1.52 & 5 & 24.4 & 15.8 & 27.9 & 19.2 & 21.8 \\
\hline AVE. & & 50.3 & 46.8 & 48.8 & 51.8 & \\
\hline
\end{tabular}

Mean Weight Loss Under pH4 Conditions $49.4 \pm 28.0$ wt\% 
TABLE 56. Weight Loss of Canister 113 Samples Under Soxhlet Conditions wt\% Loss

\begin{tabular}{|c|c|c|c|c|c|c|}
\hline \multirow{2}{*}{\multicolumn{2}{|c|}{ Core Height }} & \multicolumn{4}{|c|}{ Core Depth, cm (in.) } & \multirow[b]{2}{*}{ Ave. } \\
\hline & & $\begin{array}{l}0-7.6 \\
(0-3) \\
\end{array}$ & $\begin{array}{l}7.6-15.2 \\
(3-6)\end{array}$ & $\begin{array}{c}15.2-22.9 \\
(6-9) \\
\end{array}$ & $\begin{array}{c}22.9-30.5 \\
(9-12) \\
\end{array}$ & \\
\hline 0 & 0 & 2.04 & 2.00 & 2.65 & 2.52 & 2.30 \\
\hline 0.30 & 1 & 1.88 & 2.05 & 2.29 & 2.05 & 2.07 \\
\hline 0.61 & 2 & 2.00 & 2.21 & 2.32 & 2.92 & 2.36 \\
\hline 0.91 & 3 & 2.52 & 2.06 & 2.28 & 2.21 & 2.27 \\
\hline 1.21 & 4 & 2.89 & 3.11 & 2.51 & 2.59 & 2.78 \\
\hline 1.52 & 5 & 2.37 & 2.24 & 2.71 & 2.30 & 2.44 \\
\hline AVE. & & 2.28 & 2.28 & 2.46 & 2.43 & \\
\hline
\end{tabular}

Mean Weight Loss Under Soxhlet Conditions $2.36 \pm 0.33$ wt\%

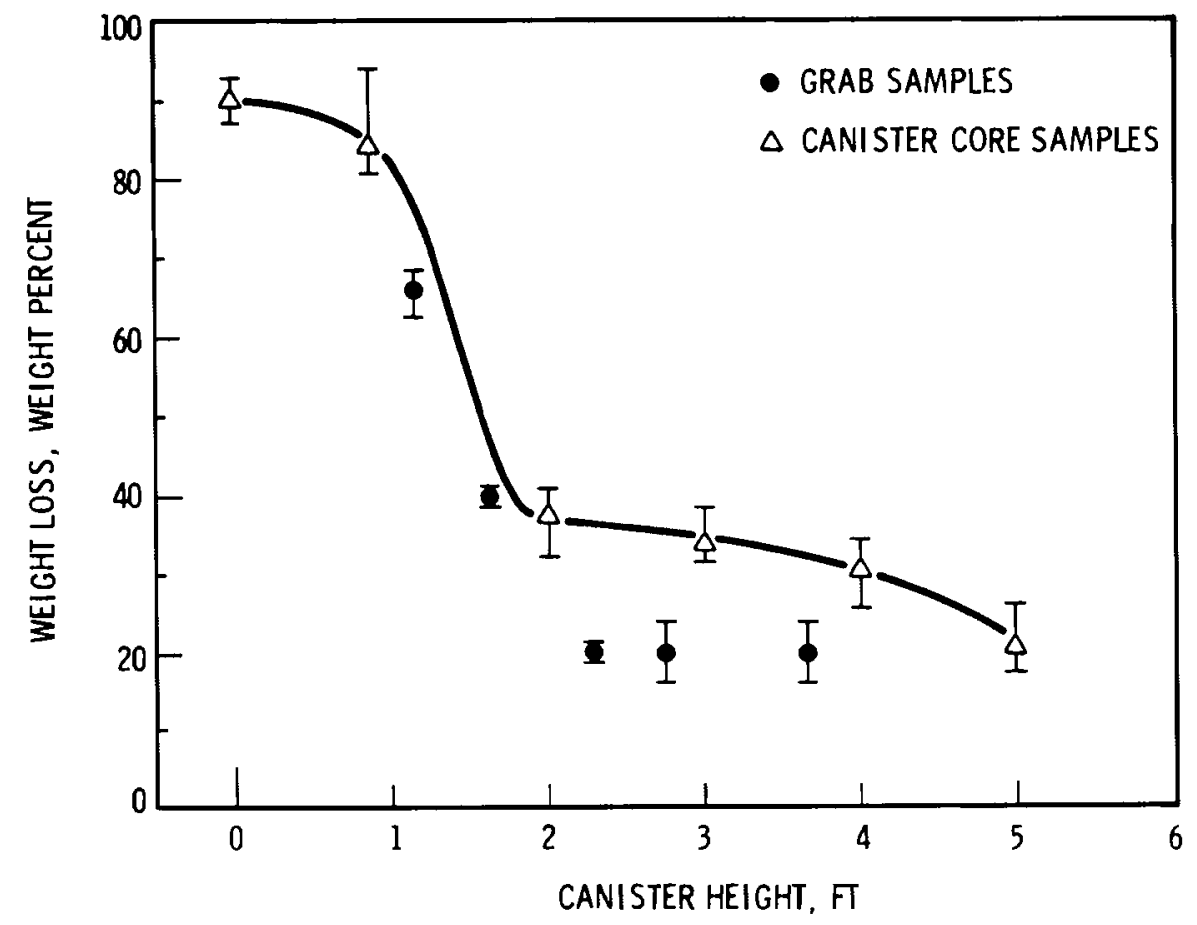

FIGURE 23. Weight Loss of CFCM-8 Glass Under pH4 Conditions 


\section{DISCUSSION}

Over $65,000 \mathrm{~kg}$ of simulated defense waste glass was produced at PNL between March 1979 and March 1980. The three vitrification processes used, SC/ICM, LFCM and SC/CFCM, involve differences in equipment design and operation. These factors contribute to variations in the final glass product. Table 57 summarizes glass product information collected during the various melting trials. These results will be discussed in relation to liquid feed composition, melting process, and product durability for canisters filled by the three vitrification processes.

\section{LIQUID FEED COMPOSITION}

The three vitrification processes all melt a mixture of waste and glassforming constituents. The simulated waste component was prepared by mixing chemicals in water at specified concentrations. The behavior of the liquid waste feed in-process equipment was a function of solubility, density and particle sizes of the individual waste components.

Liquid feed samples were collected and analyzed during three SC/CFCM melting trials. This data listed in Tables 37,42 and 50 is summarized in Table 58. Although the feed was prepared to the defined TDS waste composition, the concentration was adjusted as necessary to improve calciner throughput capacity.

Under controlled pilot-plant conditions at PNL where industrial-grade chemicals are used, up to $10 \%$ variation is normally encountered (in individual feed components). Changes in chemical suppliers can produce larger variations if the materials are not assayed prior to use. This situation was encountered with ferric hydroxide. Coarse insoluble components such as zeolite and $\mathrm{MnO}_{2}$ are difficult to deliver to the process at a constant rate and may present sampling problems. Zeolite, which is the main source of $\mathrm{SiO}_{2}$ in the waste, varied considerably. However, in terms of the waste and resulting waste glass composition, these fluctuations were not significant. Another example of difficulty in feeding dense insoluble constituents is $\mathrm{MnO}_{2}$. Melting trial CFCM-6 


\section{TABLE 57. Data Summary for Melting Trial Samples}

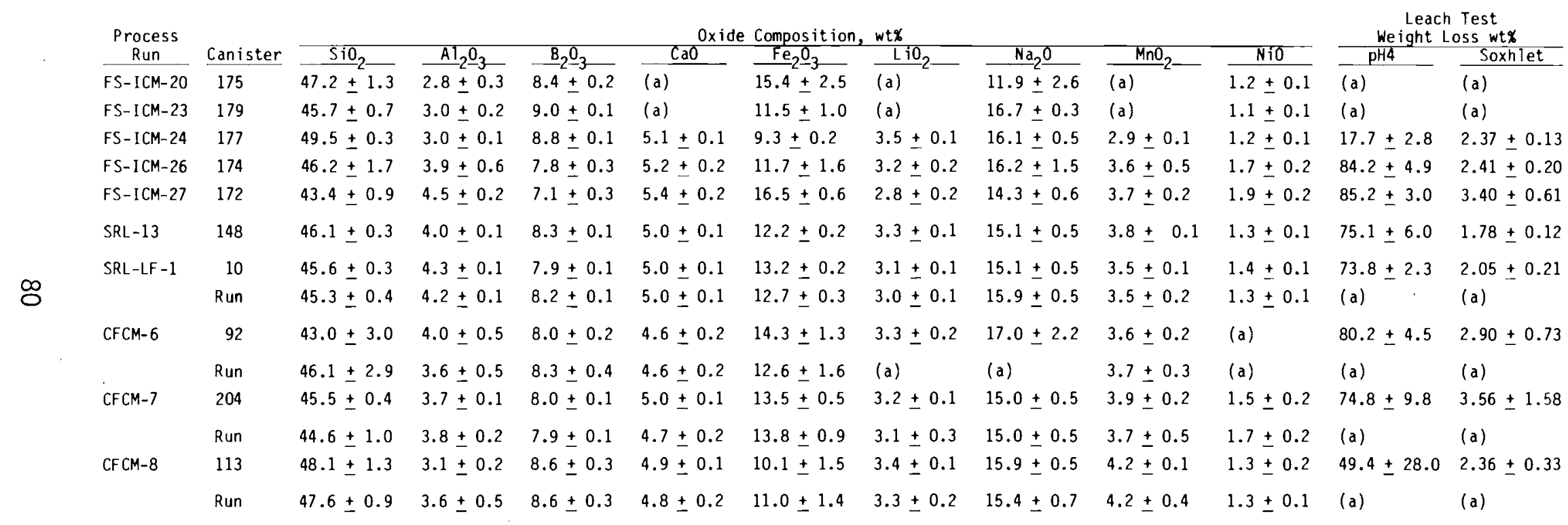

(a) Data not reported. 
TABLE 58. Summary of SC/CFCM Liquid Feed Analysis, g Oxide/L Feed

\begin{tabular}{|c|c|c|c|c|c|c|c|c|c|c|c|c|}
\hline \multirow{2}{*}{\multicolumn{3}{|c|}{ Melting Trial }} & \multicolumn{8}{|c|}{ g $0 x$ ide/L Feed } & \multirow{3}{*}{$\mathrm{pH}$} & \multirow{3}{*}{$\begin{array}{c}\text { Specific } \\
\text { Gravity, } \\
\text { g/cc }\end{array}$} \\
\hline & & & \multirow{2}{*}{$\begin{array}{l}\text { Total } \\
\text { 0xides } \\
46.0\end{array}$} & \multirow{2}{*}{$\frac{\mathrm{SiO}_{0}}{3.3} 2$} & \multirow{2}{*}{$\begin{array}{l}\frac{\mathrm{Fe}_{2} \mathrm{O}_{3}}{24.2}\end{array}$} & \multirow{2}{*}{$\frac{\mathrm{Al}_{2}{ }_{2}{ }^{-}}{6.1}$} & \multirow{2}{*}{$\frac{\mathrm{Ca} 0}{1.8}$} & \multirow{2}{*}{$\frac{{ }^{\mathrm{Na}}}{0.4}-\frac{\mathrm{O}}{0.4}$} & \multirow{2}{*}{$\frac{\mathrm{MnO}}{6.4} 2$} & \multirow{2}{*}{$\frac{\mathrm{NiO}}{2.8}$} & & \\
\hline CFCM- 6 & Part I & Target & & & & & & & & & & \\
\hline & & Ave. & 39.6 & 2.6 & 18.3 & 5.7 & 1.5 & 0.2 & 6.0 & (a) & 7.7 & 1.02 \\
\hline & & Std. Dev. & 3.5 & 1.1 & 1.9 & 0.7 & 0.2 & 0.2 & 0.8 & (a) & 0.1 & 0.01 \\
\hline & Part II & Target & 55.0 & 4.0 & 28.9 & 7.3 & 2.2 & 0.6 & 7.6 & 3.4 & & \\
\hline & & Ave. & 52.0 & 3.6 & 23.3 & 7.8 & 1.8 & 0.3 & 7.5 & (a) & 7.7 & 1.03 \\
\hline & & Std. Dev. & 3.1 & 0.7 & 2.2 & 0.6 & 0.2 & 0.1 & 0.5 & (a) & 0.1 & 0.01 \\
\hline & Part III & Target & 64.0 & 4.6 & 33.7 & 8.4 & 2.6 & 0.7 & 8.9 & 3.9 & & \\
\hline & & Ave. & 57.4 & 3.4 & 27.3 & 8.4 & 2.0 & 1.0 & 6.9 & (a) & 7.6 & 1.02 \\
\hline & & Std. Dev. & 7.1 & 1.2 & 3.4 & 1.3 & 0.2 & 1.2 & 0.8 & (a) & 0.1 & 0.01 \\
\hline $\mathrm{CFCM}-7$ & & Target & 64.9 & 4.5 & 34.9 & 8.5 & 2.6 & 1.4 & 9.1 & 4.0 & & \\
\hline & & Ave. & 58.7 & 4.1 & 33.4 & 8.4 & 2.2 & 2.5 & 3.1 & 4.4 & & \\
\hline & & Std. Dev. & 2.5 & 0.6 & 1.8 & 0.6 & 0.2 & 0.3 & 1.2 & 0.2 & & \\
\hline CFCM- 8 & Part I & Target & 74.7 & 9.6 & 34.1 & 8.3 & 2.3 & 7.4 & 9.1 & 4.0 & & \\
\hline & & Ave. & 65.0 & 8.2 & 34.2 & 7.5 & 2.2 & 5.2 & 2.9 & 4.3 & 14 & \\
\hline & & Std. Dev. & 4.5 & 1.2 & 1.2 & 0.9 & 0.1 & 1.6 & 1.5 & 0.2 & & \\
\hline & Part II & Target & 65.5 & 6.5 & 34.1 & 8.3 & 2.3 & 1.2 & 9.1 & 4.0 & & \\
\hline & & Ave. & 52.9 & 3.3 & 31.6 & 7.1 & 2.0 & 1.6 & 3.3 & 3.9 & 13 & \\
\hline & & Std. Dev. & 9.4 & 1.2 & 5.9 & 1.1 & 0.2 & 0.2 & 2.0 & 0.2 & & \\
\hline
\end{tabular}

(a) Not analyzed

did not show the gross discrepancy of $\mathrm{MnO}_{2}$ results noted in CFCM-7 and CFCM-8. Since no modification of sampling procedure or feed system was made, the cause of this situation is believed to be the use of larger particle-size $\mathrm{MnO}_{2}$ source in the CFCM-7 and CFCM-8 melting trials.

Short-term variations in liquid feed composition do occasionally occur; however, they are not a normal occurrence and do not account for all variations noted in the final product. Physical properties such as $\mathrm{pH}$ and specific gravity have not been sensitive to either changes in overall feed concentration or composition. Their usefulness in process monitoring is questionable. 


\section{MELTING PROCESS}

Nuclear waste glasses are prepared by subjecting waste-glass formers to elevated temperatures for sufficient time to produce a relatively homogeneous melt. The properties of the resulting waste-glass product are then largely determined by its composition and thermal history upon cooling. Variations in glass product composition were often encountered as a result of waste/frit ratio variations. The SC/ICM and SC/CFCM attempted to maintain a constant waste/frit ratio by metering the frit at a rate determined by the liquid feed rate and the defined feed concentration. In the LFCM, premixed solid or slurry feeds were used. As a result, the composition of the SC/ICM or SC/CFCM product varied more than the LFCM product.

These types of larger compositional differences noted by chemical analysis are not to be confused with glass homogeneity. Glass homogeneity is determined by how well waste constituents are dispersed throughout the glass matrix. Longer melting times, higher melting temperatures and more convection currents within the molten glass all may improve mixing and, therefore, homogeneity. While the SC/ICM product contained a waste-enriched glass on the bottom of each canister, the bulk of the glass appeared at least as homogeneous in photomicrographs as SC/CFCM samples. Both melters were fed from the same feed/calciner system, so the difference in product homogeneity is in some way a matter of melter design and operation. The LFCM with its premixed feeds generally produced a very homogeneous product.

The glass produced by the three processes experienced significantly different thermal histories during cooling. This has a major impact on glass devitrification, the appearance of crystalline phases. The electric melters, LFCM and CFCM, pour a molten glass stream into a canister. All grab samples collected at this point were amorphous with no crystalline phases present. Upon cooling, acmite crystals with a wide range of sizes were formed within the glass. Similarly, the bulk of the ICM glass contained acmite crystals. The impact of this phase on durability has not been established; however, it has led to microcracking in many samples. ICM canisters, in addition, contain a spinel crystal accumulation on the bottom. How much this segregation is 
due to thermal history is not known. Temperature data for the various canisters is being processed and will be useful in explaining variations in crystals sizes and quantities.

PRODUCT DURABILITY

The chemical durability of a nuclear-waste glass is determined by many factors, including composition, surface area and the presence of secondary phases. Various leach tests are used to measure durability under specific environmental conditions. Leach testing procedures vary considerably and are a source of much debate regarding their representation of actual waste storage conditions. Only the pH4 and soxhlet tests were used to evaluate the durability of melting trial samples. The average results for each canister are listed in Table 57 .

The pH4 leach results were considerably more sensitive to glass compositional changes than soxhlet results. Canisters 177 (SC/ICM) and 113 (SC/CFCM) had the lowest pH4 weight losses as well as the lowest waste content. In addition, canister 113 had a large waste content gradient which produced the large pH4 standard deviation. In these cases, soxhlet values were largely unaffected, remaining in the range of 2.05 to $3.56 \mathrm{wt} \%$ 1oss. Although durability is a function of glass composition, the standard deviations of chemical analys is did not correspond to leach data standard deviations. The average standard deviation of $\mathrm{pH} 4$ and soxhlet weight loss data were $13.9 \%$ and $16.5 \%$, respectively.

In only one canister, 204, unusually large standard deviations were encountered for both $\mathrm{pH} 4$ and soxhlet leach results. The composition of the CFCM product was very consistent with only minor variations. While extensive microcracking was present in this canister, a similar condition in canister 172 (Figure 8) did not result in large leach data variations. 

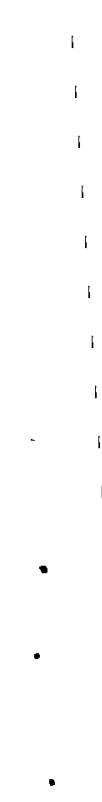


\section{CONCLUSIONS AND RECOMMENDATIONS}

Three vitrification processes produced over $67,000 \mathrm{~kg}$ of simulated defense nuclear waste glass from March 1979 to August 1980. Feed and product samples during this period were collected and analyzed. Operational changes and unexpected events are a normal part of extended melting trials. The analys is of feed and product data during this period was useful in identifying process parameters most signficantly affecting the final product. We make the following conclusions:

\section{LIQUID FEED}

Liquid feed samples are necessary to determine the impact of mixing and feed system operation on the waste composition. The sampling procedure must, however, collect a representative sample to be most useful. During extended melting trials, the normal variation of constituents may be as high as $10 \%$ of the constituent concentration. Feed samples every $250 \mathrm{~kg}$ of glass production would provide adequate information for process monitoring. Impurities may also be unknowingly associated with some of the waste components used in feed preparation.

\section{GLASS PRODUCT}

The composition of the final product largely determines its physical and chemical properties. The product composition is determined by the waste composition and the ability of the process to maintain a constant waste/frit ratio. Experience at PNL shows that the waste content of the glass during a melting trial is within $\pm 3.6 \%$ of the defined $28 \%$ waste oxide content. A11 trials together were within $\pm 6 \%$ of the target. Those processes requiring the simultaneous control of liquid waste and frit feed rates, SC/ICM and SC/CFCM, had the largest composition variations. Glass compositions tolerating this variation without property changes would produce the most consistent product.

Crystals are a common feature of the defense waste glass cooled in canisters. The complex interaction of glass composition and cooling rate must be 
understood to anticipate the appearance of crystalline phases and their impact on product properties. Acmite crystals frequently found in SC/ICM and SC/CFCM product resulted occasionally in considerably glass microcracking. The cause of the spinel (waste) accumulation in the bottom of SC/ICM canisters has not been positively identified.

Waste oxides can be reduced in the waste glass melt and settle out as metallic buttons. While organics in the "modified TDS" composition were effective in reducing metals in the SC/ICM process, additional work is necessary to determine the tendency for this to occur in the ceramic melters.

The chemical durability of glass samples under pH4 and soxhlet conditions does not appear related to the particular process or its stable operation. The SC/ICM, LFCM and SC/CFCM all produced glass of comparable durability. Foaming in the ceramic melters did not appear to affect the product durability. Lowering waste content of the defense waste glass increased pH4 durability but did not affect soxhlet durability. The average variation in leach test data as a percentage of the mean leach test results was considerable, $14.0 \% \pm 17.8 \%$ for pH4 and $17.7 \% \pm 12.8 \%$ for the soxhlet test. Since this shows a little relationship to corresponding composition variation, care must always be exercised in product evaluation on the basis of leach test results.

\section{RECOMMENDATIONS}

We make these recommendations for future equipment melting trials:

- Feed and product sampling should be an integral part of every melting trial with data analysis based on computer control charge programs to deal with inherent process variations.

- Sampling frequency can be reduced to two samples per feed makeup or every $250 \mathrm{~kg}$ of glass produced. The sampling procedure should be improved to make each sample more representative.

- The sampling of ceramic melter-produced glass from the pour spout every $250 \mathrm{~kg}$ of production is adequate for chemical analysis and leach testing. 
- Crystallization studies need to be carried out on glasses in a range of at least \pm 6 wt\% of waste from the defined composition to anticipate devitrification effects.

- The impact of organic compounds on glass properties and metal reduction from the melt should be examined.

- Methods to eliminate the enrichment of waste in the bottom of SC/ICM canisters should be investigated. 


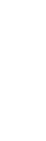




\section{REFERENCES}

Bauer, P. and P. Hackl. 1978. The Use of MOSUMS for Quality Control. Volume 20(4), pp. 431-436.

Blair, H. Thomas. 1979. In-Can Melting Process and Equipment Development from 1974 to 1978. PNL-2925, Pacific Northwest Laboratory, Richland, WA 99352.

Bonner, W. F. et. al. 1976. Spray Solidification of Nuclear Waste. BNWL2059, Pacific Northwest Laboratory, Richland, WA 99352.

Buelt, J. L. and C. C. Chapman. 1978. Liquid-Fed Ceramic Melter: A General Description Report. PNL-2735, Pac if ic Northwest Laboratory, Richland, WA 99352.

Buelt, J. L. and C. C. Chapman. 1979. Slurry Feeding of Nuclear Waste to an Electric Glass Melter. PNL-SA-7571, Pacific Northwest Laboratory, Richland, WA 99352.

Chick, L. A. et. al. 1980. The Effects of Composition on Properties in an Eleven Component Nuclear Waste-Glass System. PNL-3188, Pacific Northwest Laboratory, Richland, WA 99352.

Dierks, R. D. 1980. The Design of a $100 \mathrm{~kg} / \mathrm{h}$, Direct Calcine-Fed Electric Melter for Nuclear Waste Vitrification. PNL-3387, Pacific Northwest Laboratory, Richland, WA 99352.

McElroy, J. L., J. E. Mendel, W. F. Bonner and M. H. Henry. 1979. Quarterly Progress Report - Research and Development Activities - High-Level Waste Immobilization Program: April Through June 1979. PNL-3050-2, Pacific Northwest Laboratory, Richland, WA 99352. 


\section{DISTRIBUTION}

No. of

Copies

OFFSITE

A. A. Churm

DOE Chicago Patent Group

9800 South Cass Avenue

Argonne, IL 60439

2 R. Y. Lowrey

DOE Albuquerque Operations Office

P.0. Box 5400

Albuquerque, NM 87185

A. L. Taboas

DOE Albuquerque Operations Office

P.0. Box 5400

Albuquerque, NM 87185

S. A. Mann

DOE Chicago Operations and Region Office

Argonne, IL 60439

\section{J. 0. Neff}

Department of Energy

Columbus Program Office

505 King Avenue

Columbus, $\mathrm{OH} 43201$

W. E. Mott

DOE Division of Environmental Control Technology

Washington, DC 20545

J. P. Hamric

DOE Idaho Operations Office

550 2nd St.

Idaho Falls, ID 83401

J. W. Peel

DOE Idaho Operations Office

550 2nd St.

Idaho Falls, ID 83401
No. of

Copies

J. B. Whitsett

DOE Idaho Operations Office

550 2nd St.

Idaho Falls, ID 83401

C. R. Cooley

DOE Nuclear Waste Management Programs

NE-331, GTN

Washington, DC 20545

G. H. Daly

DOE Nuclear Waste Management Programs

NE-322, GTN

Washington, DC 20545

J. E. Dieckhoner

DOE Nuclear Waste Management Programs

NE-321, GTN

Washington, DC 20545

C. H. George

DOE Nuclear Waste Management Programs

NE-330, GTN

Washington, DC 20545

C. A. Heath

DOE Nuclear Waste Management Programs

NE-330, GTN

Washington, DC 20545

M. L. Lawrence

DOE Nuclear Waste Management

Programs

NE-340, GTN

Washington, DC 20545 
No. of

Copies

D. J. McGoff

DOE Nuclear Waste Management

Programs

NE-320, GTN

Washington, DC 20545

S. Meyers/R. Romatowski

DOE Nuclear Waste Management

Programs

NE-30, GTN

Washington, DC 20545

G. Oertel

DOE Nuclear Waste Management

Programs

NE-320, GTN

Washington, DC 20545

A. F. Perge

DOE Nuclear Waste Management

Programs

NE-30, GTN

Washington, DC 20545

R. W. Ramsey, Jr.

DOE Nuclear Waste Management Programs

NE-301, GTN

Washington, DC 20545

V. Trice

DOE Nuclear Waste Management

Program

NE-30, GTN

Washington, DC 20545

D. L. Vieth

DOE Nuclear Waste Management

Programs

NE-332, GTN

Washington, DC 20545

2 S. W. Ahrends

DOE Oak Ridge Operations Office

P.0. Box E

Oak Ridge, TN 37830
No. of

Copies

D. E. Large

DOE Oak Ridge Operations Office P.0. Box E

Oak Ridge, TN 37830

S. G. Harbinson

DOE San Francisco Operations

Office

1333 Broadway

Oakland, CA 94612

W. B. Wilson

DOE Savannah River Operations Office

P.0. Box A

Aiken, SC 29801

R. P. Whitfield

DOE Savannah River Operations Office

P.0. Box A

Aiken, SC 29801

J. B. Martin

Division of Waste Management Nuclear Regulatory Commission

Washington, DC 20555

D. B. Rohrer

Division of Waste Management

Nuclear Regulatory Commission

Washington, DC 20555

R. D. Smith

Division of Waste Management

Nuclear Regulatory Commission

Washington, DC 20555

R. E. Cunningham

Office of Nuclear Safety

Materials and Safeguards

Nuclear Regulatory Commission

Room 562, 7915 Eastern Avenue

Silver Springs, MD 20910

27 DOE Technical Information Center 
No. of

Copies

J. A. Buckham

Allied-General Nuclear Services P.0. Box 847

Barnwell, SC 29812

A. Williams

Allied-General Nuclear Services P.0. Box 847

Barnwell, SC 29812

J. H. Kittel

Argonne National Laboratory

Office of Waste Management

Programs

9700 South Cass Avenue

Argonne, IL 60439

M. J. Steindler/L. E. Trevorrow Argonne National Laboratory

9700 South Cass Avenue

Argonne, IL 60439

W. Carbiener

Battelle Memorial Institute

Office of Nuclear Waste

I solation

505 King Avenue

Columbus, $\mathrm{OH} 43201$

Beverly Rawles

Battelle Memorial Institute

Office of Nuclear Waste

I solation

505 King Avenue

Columbus, $\mathrm{OH} 43201$

Research Library

Battelle Memorial Institute

505 King Avenue

Columbus, $\mathrm{OH} 43201$

R. Maher, Program Manager Waste Management Programs

Savannah River Plant

E. I. Du Pont de Nemours \& Co.

Aiken, SC 29801
No. of

Copies

M. D. Boersma

E. I. Du Pont de Nemours \& Co.

Savannah River Laboratory

Aiken, SC 29801

R. G. Garvin

E. I. Du Pont de Nemours \& Co.

Savannah River Laboratory

Aiken, SC 29801

D. L. McIntosh

E. I. Du Pont de Nemours \& Co.

Savannah River Laboratory

Aiken, SC 29801

A. L. Ayers

$E G$ \& G Idaho

P.0. Box 1625

Idaho Falls, ID 83415

R. Williams

Electric Power Research Institute

3412 Hillview Avenue

Palo Alto, CA 94304

2 Environmental Protection Agency

Technological Assessment

Division (AW-559)

Office of Radiation Programs

U.S. Environmental Protection Agency

Washington, DC 20460

J. R. Berreth

Exxon Nuclear Idaho

P.0. Box 2800

Idaho Falls, ID 83401

G. L. Ritter

Exxon Nuclear I daho

P.0. Box 2800

Idaho Falls, ID 83401 
No. of

Copies

3 J. Campbe 11

Lawrence Livermore Laboratory

P.0. Box 808

Livermore, CA 94550

R. Roy

202 Materials Research Laboratory

Pennsylvania State University University Park, PA 16802

3 A. L. Lotts

Oak Ridge National Laboratory P.0. Box $X$

Oak Ridge, TN 37830

2 A. B. Martin

Rockwell International

Energy Systems Group

8900 DeSoto Avenue

Canoga Park, CA 91304

Paul Hagen

Chemical Operations

Rockwell International

Rocky Flats Plant

P.0. Box 464

Golden, CO 80401

E. Vejvoda, Director

Chemical Operations

Rockwe 11 International

Rocy Flats Plant

P.0. Box 464

Golden, CO 80401

R. G. Kepler

Organic and Electronic

Dept. 5810

Sandia Laboratories

Albuquerque, NM 87185

P. B. Macedo

Keane Hall

Vitreous State Laboratory

The Catholic University of America

Washington, DC 20017
No. of

Copies

L. L. Hench

Dept. of Materials Science and Engineering

University of Florida

Gainesville, FL 32611

Dr. Hayne Palmour III

2140 Burlington Engineering Laboratories

North Carolina State University

Raleigh, NC 27.607

\section{ONSITE}

4 DOE Richland Operations Office

P. A. Craig (2)

R. E. Gerton

H. E. Ransom

4 Rockwell Hanford Operations

I. E. Reep

D. D. Wodrich (3)

UNC United Nuclear Industries

F. H. Bouse

Westinghouse Hanford Company

A. G. B lasewitz

40 Pacific Northwest Laboratory

W. J. Bjorklund

W. F. Bonner

J. R. Carrel1

T. D. Chikalla

J. H. Jarrett

D. E. Knowlton

L. T. Lakey

D. E. Larson (10)

G. B. Long (3)

R. P. Marshall 
No. of

Copies

Pacific Northwest Laboratory

(contd)

J. L. McE Iroy

G. B. Mellinger

R. E. Nightingale

R. D. Peters

A. M. Platt

J. M. Rusin

D. H. Siemens

S. C. Slate

R. L. Treat

R. P. Turcotte

H. H. Van Tuy]

J. W. Wald

Technical Information (5)

Publishing Coordination (2) 
